\title{
Common patterns in pulse profiles of high-mass X-ray binaries
}

\author{
J. Alonso-Hernández ${ }^{1}$, F. Fürst ${ }^{2}$, P. Kretschmar ${ }^{3}$, I. Caballero ${ }^{4}$, and A. M. Joyce ${ }^{5}$ \\ ${ }^{1}$ Departamento de Física y Astronomía, Universidad Complutense de Madrid, Avenida de Séneca 2, Ciudad Universitaria, \\ 28040 Madrid, Spain \\ e-mail: uo251961@uniovi.es \\ ${ }^{2}$ Quasar Science Resources S.L for European Space Agency (ESA), European Space Astronomy Centre (ESAC), Camino Bajo del \\ Castillo s/n, 28692 Villanueva de la Cañada, Madrid, Spain \\ ${ }^{3}$ European Space Agency (ESA), European Space Astronomy Centre (ESAC), Camino Bajo del Castillo s/n, 28692 Villanueva de la \\ Cañada, Madrid, Spain \\ 4 Aurora Technology B.V. for European Space Agency, European Space Astronomy Centre (ESA/ESAC), Madrid, Spain \\ ${ }^{5}$ Dr. Karl Remeis-Observatory \& Erlangen Centre for Astroparticle Physics, Sternwartstr. 7, 96049 Bamberg, Germany
}

Received 12 July 2021 / Accepted 5 January 2022

\begin{abstract}
Context. X-ray pulsars are binary systems which consist of a neutron star in orbit with a mass donor (companion). In these systems the neutron accretes matter from the companion star, which creates accretion columns or hot spots on the neutron star surface and gives rise to pulsations in the X-ray light curve. The pulse profiles carry information about the accretion and magnetic field geometry. Here we present a study and classification of energy-resolved pulse profiles of a sample of X-ray pulsars, focusing on high-mass X-ray binaries.

Aims. Our goal is to perform a classification of X-ray pulsars based on their observed pulse profiles and look for correlations between this classification and their principle physical observables. The analysis pipeline is available online.

Methods. We analysed the pulse profiles of a sample of X-ray pulsars using data obtained with the X-ray Multi-Mirror Mission (XMMNewton) and the Nuclear Spectroscopic Telescope Array (NuSTAR). We fitted the energy-resolved pulse profiles with a Fourier series of up to five harmonics. We then used the energy evolution of the different Fourier components to classify the pulse profiles into groups. We investigated relationships between the pulse profile properties and other observables of the systems (e.g. orbital period, magnetic field strength, and luminosity) to study the extreme physics of these systems.

Results. The sources were divided into three groups using a classification based on the shape, the dominance of the fitted Fourier harmonics, and their respective evolution with energy. We do not find a conclusive correlation between the pulse profile shapes or groups and other parameters of the systems. However, a weak trend was found when comparing our classification to the sources' locations in the spin period-orbital period diagram. Further studies are required to confirm this trend.

Conclusions. Despite the large variety of pulse profiles of the X-ray pulsars, we found that with our approach clear categories emerge which we use to classify their behaviour as a function of energy. As we do not find a clear relationship between our classification scheme and other parameters, like the luminosity, the magnetic field strength, or the orbital and spin periods, we conclude that X-ray pulse profiles are influenced by other hidden variables.
\end{abstract}

Key words. pulsars: general - X-rays: binaries - accretion, accretion disks

\section{Introduction}

Accreting X-ray pulsars are X-ray binary systems in which a highly magnetized neutron star, with a magnetic field strength typically in the range $10^{11-13} \mathrm{G}$, accretes matter from its companion star. The mass donors are mainly stars with masses $>10 M_{\odot}$ (Liu et al. 2006), making the systems high-mass X-ray binaries (HMXBs), but some also exist in low- or intermediate-mass Xray binaries (LMXBs, IMXBs) with a companion star of a few solar masses or less (Liu et al. 2007).

The mass transfer can take place in different ways, depending on the nature of the mass donor and the size of the binary orbits. For lower mass systems and some HMXBs with neutron stars in very tight orbits, mass transfer will take place via Roche-lobe overflow (Campana \& Di Salvo 2018; Brumback et al. 2020). In a large group of $\mathrm{HMXBs}$ the mass donor will be an early-type star losing copious amounts of matter through line-driven winds. Neutron stars in close orbits around their companions can accrete efficiently from this wind and become bright X-ray sources. For a recent review of these systems see Martínez-Núñez et al. (2017). Some systems may mix Roche-lobe overflow and wind accretion (El Mellah et al. 2019). Finally, there is the sub-class of Be X-ray Binaries (BeXRBs) in which the companion is a Beor Oe-type star (i.e. an early-type star with a large circumstellar disk) (Nixon \& Pringle 2020). The neutron star in these systems can be on a wide, eccentric orbit. When the neutron star is close enough to the mass donor and other conditions are met, X-ray outbursts can occur (Negueruela \& Okazaki 2001; Okazaki \& Negueruela 2001). In many systems, both regular weaker outbursts and irregular giant outbursts have been observed. For an overview of BeXRBs and their behaviour see Reig (2011).

Regardless of the mode of accretion, at a certain distance from the neutron star, typically two to three orders of magnitude larger than the neutron star radius (see Kretschmar et al. 2019a, for a recent overview of interactions at the magnetosphere), the magnetic field will begin to dominate the movement of the accreted plasma, which will then follow the field lines towards the magnetic poles. Assuming a dipolar magnetic field, 
two accretion columns at the magnetic poles of the neutron star will be produced. If there is a misalignment between the magnetic and the rotation axis of the neutron star, we will observe the system as an X-ray pulsar, as the X-ray emitting region rotates in and out of our line of sight (Pringle \& Rees 1972).

In the simplest picture of a dipolar magnetic field with axial symmetric emission pattern, we would expect pulse profiles with almost sinusoidal shapes; such profiles can be found in accreting millisecond pulsars (Poutanen et al. 2009), which have lower magnetic fields. However, the majority of accreting X-ray pulsars have profiles that are more complex and frequently show multipeaked patterns, especially at low energies, and often exhibit a strong energy dependence (see e.g. Kanno 1980, for early examples). Typically, each source shows its own unique behaviour, making the pulse profiles 'fingerprints' of their sources, stable over long periods (see, e.g. Fig. 9 in Kretschmar et al. 2021). There are also cases of significant variations in observed profiles of transient sources during an outburst, where the pulse profiles can dramatically change their looks and behaviour (see e.g. Brumback et al. 2021; Camero Arranz et al. 2007; Cappallo et al. 2019).

The changes in profile shapes with energy indicate corresponding changes in the emission geometry of the accretion columns. Studying this energy dependence contains information about the physical processes inside the accretion column, like Comptonization, Bremsstrahlung and cyclotron radiation (Becker \& Wolff 2005, 2007; Wolff et al. 2016). For disk-fed pulsars there will also be reprocessing of soft X-rays in the large accretion disk (Hickox et al. 2004; Hickox \& Vrtilek 2005; Brumback et al. 2020, 2021). In addition, emission lines of heavy elements such as iron, produced by fluorescence stimulated in the accretion disk or in the circumstellar material, can produce significant effects at the corresponding line energies in the spectra and pulse profiles.

Attempts to relate theoretically predicted beam patterns to pulse profiles of accreting X-ray pulsars have been undertaken since the early days of X-ray astronomy (e.g. Basko \& Sunyaev 1975; Kanno 1980; Wang \& Welter 1981). Over time, the critical importance of including gravitational light bending effects (Riffert \& Meszaros 1988; Riffert et al. 1993) and allowing for asymmetrical emission (Leahy 1991; Kraus et al. 1995) in such model efforts has been demonstrated. Such approaches have been used by various authors to try and model the emission geometry for specific sources (e.g. Bulik et al. 1995; Leahy 2004; Caballero et al. 2011; Sasaki et al. 2012; Iwakiri et al. 2019), frequently limited to specific observations or energy ranges.

As described in detail in Falkner (2018), a full description of the profiles based on the physical properties of the neutron star and the conditions within the accretion column is extremely complex. Multiple non-linear effects have to be taken into account: plasma density and temperature, energy- and magneticfield dependent cross-sections in radiation transport, relativistic beaming, and gravitational light bending. Despite all efforts, the a priori constraints on these effects are very limited, which means that large degeneracies between different parameters influencing the profiles can appear, leading to multiple geometrically different solutions (e.g. Caballero et al. 2011). The picture is somewhat different for sources where the emission can be described by surface hotspots without significant columns, for example in accreting millisecond pulsars. In this case the accurate analytical approximation to the Schwarzschild metric at $R \geq 2 r_{\mathrm{g}}$ derived by Beloborodov (2002) can be used (Poutanen $\&$ Beloborodov 2006), as demonstrated by the recent Neutron star Interior Composition Explorer (NICER) results (Riley et al. 2019).

A different and complementary approach to the characterization of pulse profiles in accreting X-ray pulsars is a decomposition of the overall profile into simple, possibly energydependent components without any a priori connection to the complex emission and radiation transport physics. While such an approach cannot yield direct insights into the underlying physics, it avoids any entanglement in possibly degenerate parameter dependencies and is conceptually not that different from the widespread approach of describing broadband spectra of Xray sources with simple phenomenological models. Such an approach can then also be used in an easy and automatic manner across multiple sources, focusing on broad similarities and differences between their pulse profiles.

As has been demonstrated before (e.g. Finger et al. 1999; Camero Arranz et al. 2007), describing pulse profiles based on their sinusoids or Fourier frequencies provides a fairly precise fit of the pulse profile and its precision mainly depends on the number of harmonics of the fit. In this paper we describe a framework to study the pulse profiles of different X-ray pulsars in a range of energies with the smallest energy steps possible via a simple combination of sinusoids. Based on the properties of the components we classify the sources into different groups and investigate any dependence of the pulse profile characteristics with other properties of the system, like orbital period, luminosity, or the magnetic field of the neutron star.

We apply the framework to a small initial catalogue of a few sources. As the analysis software is public, this might be used as a basis for future observations and research related to this topic. We hope that the procedure and broad characterization provided in this way may serve to reduce the parameter space for any physical modelling effort by highlighting common trends and how they may be related to other physical properties.

The remainder of this paper is structured as follows. In Sect. 2 we explain the selection of the observations, the data analysis for timing corrections, the pulse period search, how the observed pulse profiles are obtained, and the fitting process. In addition we give an overview of the pipeline framework and explain the pipeline outputs. In Sect. 3 we present the results and give possible explanations for the emerging grouping of our source sample. Finally, in Sect. 4 we conclude the paper and provide an outlook for future work.

\section{Data analysis and scripts}

To simplify and automate the comparison between the pulse profile of a sample of sources, a pipeline was designed to obtain the normalized pulse profile resolved at different energy ranges ${ }^{1}$. The pipeline uses the Astropy (Astropy Collaboration 2013) and Stingray (Huppenkothen et al. 2019) Python packages.

\subsection{Observations}

Our analysis is based on data taken with $\mathrm{XMM}$-Newton and $\mathrm{NuS}$ $T A R$, two of the most sensitive telescopes in their respective energy bands.

The X-ray Multi-Mirror Mission (XMM-Newton; Jansen et al. 2001) is an X-ray telescope of the European Space Agency (ESA). XMM-Newton data used in this project was obtained from the EPIC-pn camera (Strüder et al. 2001), which provides

\footnotetext{
1 The pipeline is publicly available in a Github repository at https: //github.com/piexpiex/X-ray-pulsars-Pulse-Profile
} 
Table 1. Observations of X-ray pulsars used in this study.

\begin{tabular}{lcccc}
\hline \hline Source & \multicolumn{2}{c}{ OBS ID } & \multicolumn{2}{c}{ Date of observation (MJD) } \\
\cline { 2 - 5 } & XMM-Newton & NuSTAR & XMM-Newton & NuSTAR \\
\hline SMC X-1 & 0784570201 & 30202004002 & 57639.930 & 57639.899 \\
4U 1901+03 & & 90501324002 & & 58615.752 \\
& & 90502307002 & & 58549.308 \\
& & 90502307004 & & 58584.946 \\
V 0332+53 & & 80501305001 & & 58531.121 \\
& & 80102002002 & & 57223.415 \\
Cen X-3 & & 80102002010 & & 57275.946 \\
LMC X-4 & 0400550201 & 30101055002 & 53898.938 & 57356.758 \\
GRO J2058+42 & & 30102041002 & 57325.124 & 57325.048 \\
Vela X-1 & 0841180101 & & 58584.008 \\
1E1145.1-6141 & 0841000101 & 30501313004 & & 58606.868 \\
IGR J17252-3616 & 0405640901 & 30501003002 & 58606.925 & 5802002 \\
GX 301-2 & 0555200401 & 30101042002 & 55687.528 & 58687.471 \\
IGR J16393-4643 & & 30001008002 & & \\
\hline
\end{tabular}

Notes. Column (1): name of the observed source, Cols. (2) and (3): identification number of the observation for XMM-Newton and NuSTAR respectively, and Cols. (4) and (5): date of the observation in Modified Julian Date and in the time reference system of XMM-Newton and NuSTAR respectively. The sources are ordered by their spin periods $\left(P_{\text {spin }}\right)$, which are shown in Table 2.

coverage in the energy band from 0.15 to $12 \mathrm{keV}$, with a spectral resolution of $\sim 80 \mathrm{eV}$. Most of the data used here were taken in Timing or Burst mode, which provide a timing resolution of $0.03 \mathrm{~ms}$ and $0.007 \mathrm{~ms}$, respectively. The only exceptions are the observations of LMC X-4 (ObsID 0771180101) and IGR J17252-3616 (ObsID 0405640901), which were taken in Small Window mode. The XMM-Newton data were reduced with the standard software XMM-Newton Science Analysis System (SAS) v18.0 and screened for background flaring. Given the brightness of the sources, we did not find the need to filter out any flaring periods. We carefully checked for pile-up and found that this also was not an issue, in particular because we are only concerned with wide energy bands. For the timing mode data, we extracted the source light curve from an eight-column wide region centred on the brightest column. No background light curves were extracted, as the source photon dominate the whole active CCD. For the imaging mode data we used a circular region with a radius of $30^{\prime \prime}$ centred on the brightest pixel to extract the source data.

The Nuclear Spectroscopic Telescope Array (NuSTAR; Harrison et al. 2013) is the first focusing high-energy X-ray telescope. NUSTAR consists of two co-aligned telescopes with a $3-79 \mathrm{keV}$ energy bandpass. The spectral resolution (full width at half maximum, FWHM) varies from $400 \mathrm{eV}$ at $10 \mathrm{keV}$ to $900 \mathrm{eV}$ at $68 \mathrm{keV}$ with a timing resolution of $2 \mu \mathrm{s}$. The $N u S$ $T A R$ data were reduced following the standard procedure using NuSTAR Data Analysis Software (nustardas) v1.8.0 with CALDB v20201217. Source photons were extracted from circular regions centred on the brightest pixel with a typical radius between $90^{\prime \prime}-120^{\prime \prime}$, depending on the source brightness. We did not extract background photons as the sources are all orders of magnitudes brighter than the background.

All observations used are listed in Table 1. We mainly selected observations of targets observed simultaneously between XMM-Newton and NuSTAR to allow us to study the pulse profile evolution over a wider energy range. Additionally we concentrated on targets with a high signal-to-noise ratio to obtain the best possible results. The sample is far from complete and can be easily extended with the pipeline presented here. For this paper we concentrate on the small sample as a proof of concept.

\subsection{Obtaining and fitting pulse profiles}

Pulse profiles are usually presented in units of count rate (i.e. counts per second). However, because we want to compare different sources with largely different count rates and are mainly interested in the changing shape as a function of energy, we proceeded to perform a normalization of the pulse profile $(\mathrm{P}(\Phi))$ by subtracting its mean value $(\langle P\rangle)$ and dividing by the measured standard deviation $(\sigma)$, obtaining the following equation for the normalized pulse profile $\left(P^{*}\right)$ :

$P^{*}(\Phi)=\frac{P(\Phi)-\langle P\rangle}{\sigma}$.

Due to the phase periodicity of the pulse profiles, we described each profile by its Fourier series, in this case, the Fourier terms are expressed as harmonics and the fit is resolved by the Levenberg-Marquardt algorithm

$P^{\prime}(\Phi)=\sum_{j}^{n} A_{j} \cdot \sin \left(j \cdot \Phi+F_{j}\right)$,

where $P^{\prime}(\Phi)$ is the fitted pulse profile, $n$ is the number of terms in the Fourier series, $A_{j}$ is the $j$ th harmonic amplitude, and $F_{j}$ is the initial phase gap for the respective harmonic. We found that five harmonics were sufficient to correctly fit the pulse profiles. However, the harmonics with larger amplitudes, and therefore the most important for the fit, were the first three harmonics.

Our pipeline includes the following steps for data reduction and extraction:

1. Timing corrections We selected the good time intervals (GTI), defined a common start time $T_{0}$ for each dataset, and 

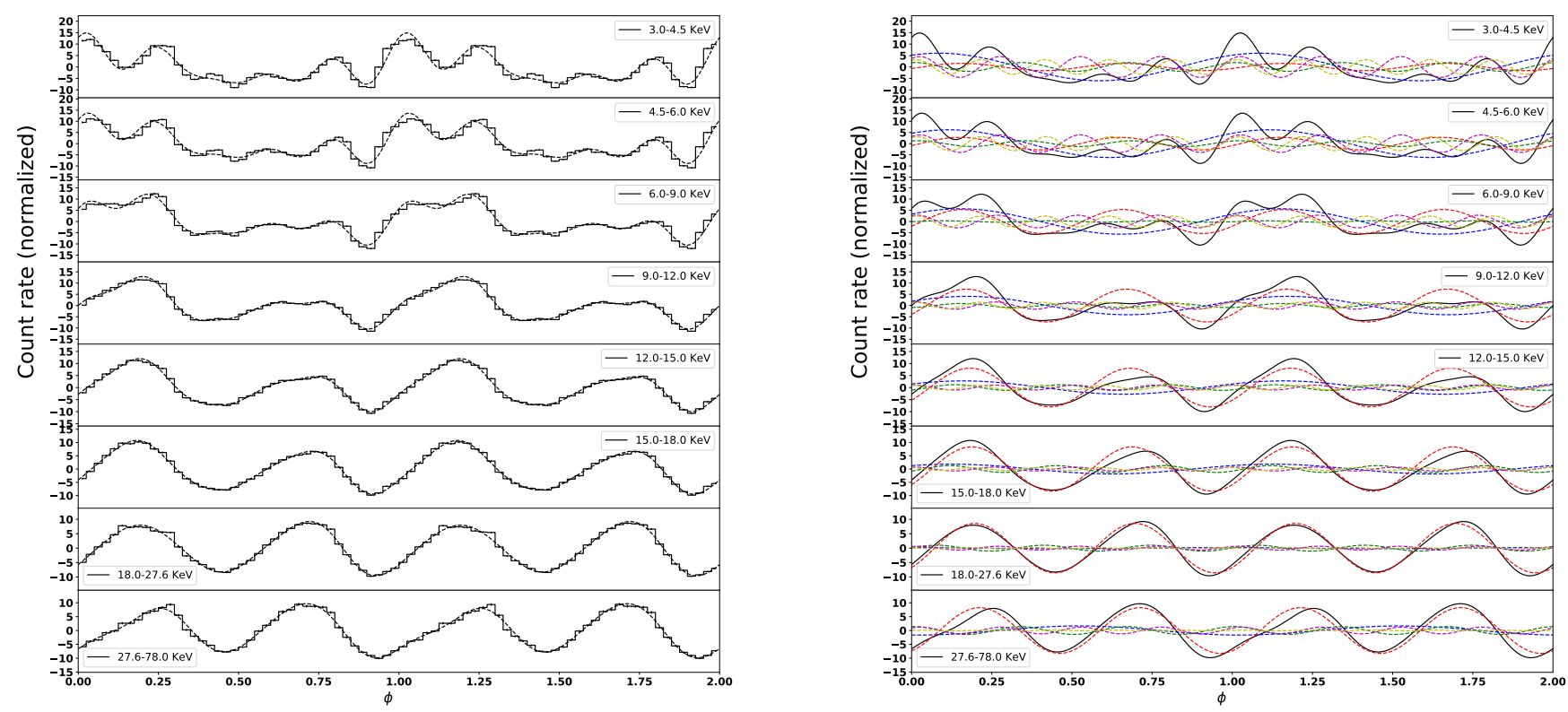

Fig. 1. Comparison of the observed energy resolved pulse profiles of Vela X-1 and their Fourier fits at different energy bands. Left: normalized pulse profiles of Vela X-1 at different energy ranges based on NuSTAR data. The measured profiles are represented by solid lines, whereas the Fourier fits are represented by dashed lines. The boundaries of the energy ranges are $[3,4.5,6,9,12,15,18,27.6$, and 78] keV. Right: overall approximate pulse profile (black solid line) and Fourier components 1-5 (coloured dashed lines: blue, red, green, purple, and yellow, respectively).
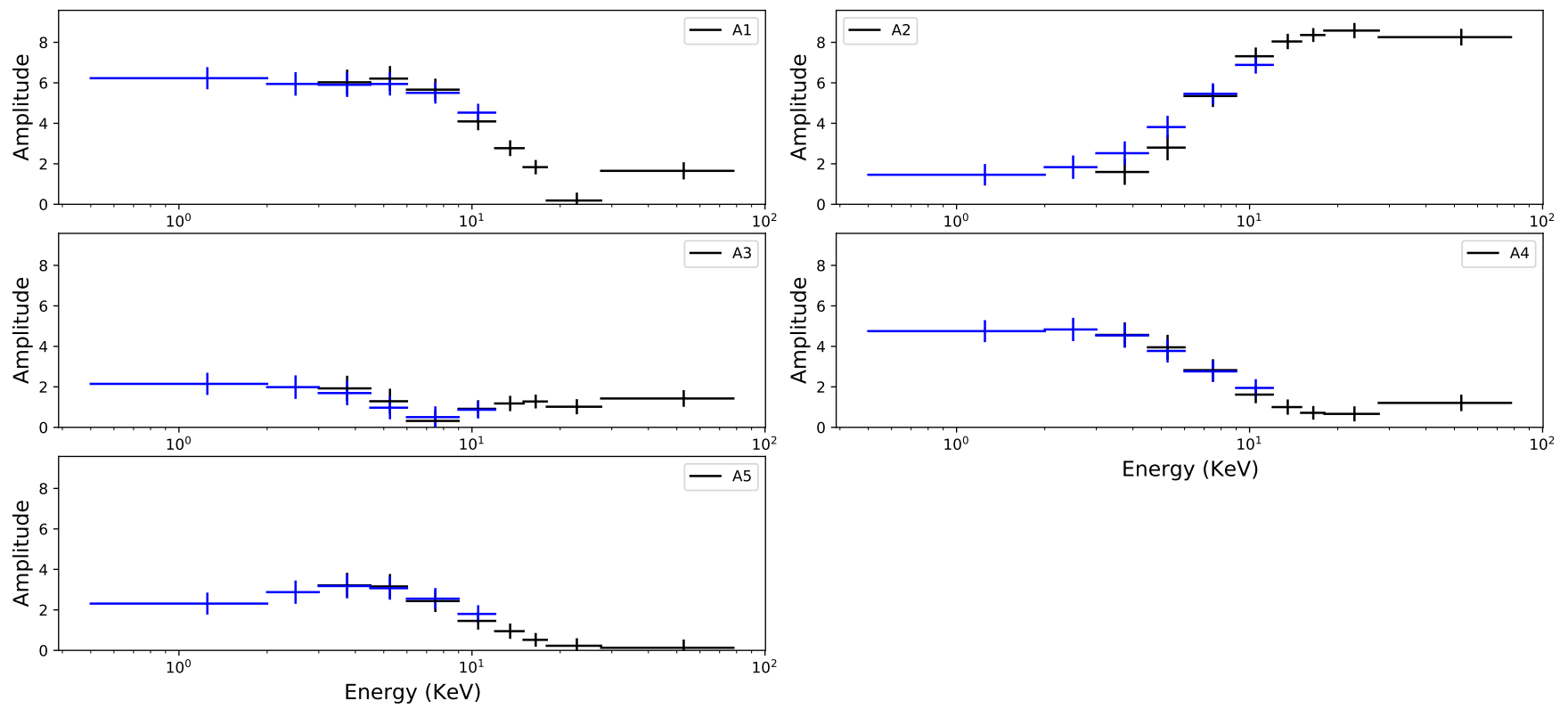

Fig. 2. Variation of the harmonic amplitudes for Vela X-1. The boundaries of the energy ranges are $[0.5,2,3,4.5,6,9,12,15,18,27.6$, and 78$] \mathrm{keV}$. $X M M$-Newton data is represented by blue markers and NUSTAR data is represented by black markers.

corrected the arrival times for each photon to the binary system barycentre using the correspondening ephemeris.

2. Search of the pulse period We used the epoch folding statistic around the expected pulse period (Leahy et al. 1983). We selected each pulse period as the maximum value of the epoch folding distribution, estimating its uncertainty as the half of the full width at half maximum (FWHM/2) of a Lorentzian curve fitted to the distribution. In addition, we used the $Z^{2}$ statistic to check the obtained value. We did not consider pulse period derivates due to the short periods of the observations and the accuracy needed for this study.
3. Energy-resolved pulse profiles We binned the photons according to their energy into $N$ energy bands and calculated a pulse profile for each energy band, based on the pulse period obtained in the previous step.

4. Normalized and fitted pulse profiles We normalized the energy-resolved pulse profiles using Eq. (1) and fitted them by Fourier series as in Eq. (2) (Fig. 1).

5. Extract parameter We extracted the amplitude for each harmonic of the Fourier series as a function of energy to study the energy dependence and compare sources with each other (Fig. 2). 
Figure 1 shows an example of the energy-resolved pulse profiles; in this case the pulse profiles correspond to the NuSTAR observation of Vela X-1. There is a change in the shape of the pulse profile with the energy and that produces a drastic change in the amplitudes of the harmonics.

These changes are also shown in Fig. 2, which shows the variation of the amplitudes of the Fourier harmonics. The first two harmonics are dominant (as for most of the sources). Moreover, they show a clear change as a function of energy, with the amplitude of the first harmonic decreasing, while the amplitude of the second harmonic is increasing. Higher order harmonics do not usually show a clear trend as a function of energy and might have larger uncertainties, due to the fact that they fit small effects of the pulse profiles.

The shape of the peaks is given by the difference between the phases and the intensities of the harmonics. In some cases where the dominant harmonics are in phase $\left(\Delta \Phi_{0} \simeq 0\right)$ and the two first harmonics have a large amplitude, two intense and thin peaks appear, contrary to the cases where the peaks are not in phase $(\Delta \Phi \simeq 0)$, only one wide or truncated peak or some irregular peaks are visisble.

The results presented in this work have been obtained with a number of 40 bins in the pulse profiles phases. However, a change in this parameter does not lead to a significant change in the pulse profile shapes or the harmonic amplitudes, even in the cases with complex pulse profiles shapes or low signal-to-noise ratios.

\section{Results}

We present our results of the classification of X-ray pulsars in pulse profile categories and the possible relationships between their pulse profiles and important physical observables.

\subsection{Pulse profile classification}

The sources were classified according to their pulse profile shape, the amplitude and phase of their Fourier components, and their evolution with energy. In some cases the shape of the pulse varies drastically between lower energies and higher energies. Even so, in a few cases the shape only shows small variations in the different energy ranges. Therefore, we performed the classification manually by observing common trends in the different sources.

The most decisive aspect of our classification is the variation of the amplitudes of the Fourier harmonics with the energy. There are sources with similar trends and pulse profiles, whereas other sources present some of these characteristics that are rather different from those common in their respective groups. The groups were named according to the harmonic that presents the largest increase in amplitude with energy and dominates at higher energies. This naming scheme is completely phenomenological and might not reflect physical similarities.

Type 0. The most important characteristic of this type is that it shows little variation or some noise in certain energy ranges. In this case the harmonics do not exhibit clear variations with the energy, and the relationship between the amplitudes remains almost constant in all the energy ranges that we have studied. The three sources classified as type 0 present these characteristics and pulse profile shapes with some similarity: truncated peak for IGR J16393-4643 and V 0332+53 (OBS ID = 80102002004) and a thick peak for LMC X-4 and IGR J17252-3616.
Type 1. This group presents a dominance of the first harmonic, especially at higher energies where the profile shows a single peak. However, at lower energies these sources present a great variety of shapes. In this case the first harmonic increases its amplitude with energy while the second and third harmonic decrease their amplitude. 4U 1901+03 (OBS ID = 90502307002 and OBS ID = 90501305001) and Cen X-3 show these characteristics. However, 1E1145.1-6141 has also an increase in the amplitude of the 2nd harmonic, although the 1st harmonic is dominant. Moreover, for this group, most of the sources have an important change in the shape of their pulse profile in the range $\sim 3-15 \mathrm{keV}$.

Type 2. This group of sources is described by the dominance of the first two harmonics. In addition, they present an increase in the second harmonic amplitude and in most of the sources a decrease in the first harmonic amplitude with energy, which causes a two-peaked structure in most of the sources at higher energies, with the exception of GRO J2058+42. At low energies they show complex structure with two or more peaks, SMC X-1, Vela X-1, V 0332+53 (OBS ID = 80102002002 and OBS ID = 80102002010) and GX301-2, even though 4U 1901+03 $($ OBS ID $=90501324002$ and OBS ID $=90502307004)$ only show one peak in each pulse profile. On the other hand, the case of GRO J2058+42 is more complicated, it shows an increase in both first and second harmonics and shows only one peak at higher energies, although it shows complex shapes at lower energies as well. Furthermore, most of the sources in this group have an important change in the shape of their pulse profile in the range $\sim 3-30 \mathrm{keV}$.

Figure 3 shows a sample of energy-resolved pulse profiles, two for each type. It is remarkable that type 0 sources show little variation in all the energy ranges, whereas types 1 and 2 have notable changes in the different energy ranges.

On the other hand, the variations in the amplitudes of their respective first three harmonics are reflected in Fig. 4. The variations of the sources of type 1 and 2 have identifying tendencies, whereas the sources of type 0 have the common characteristic of not presenting large variations in the different energy steps.

\subsection{Luminosity and magnetic field}

We studied possible correlations between the pulse profile category, the luminosity, and the magnetic field strength of each source, using the data shown in Table 2.

We plot our results in Fig. 5, which shows the luminositymagnetic field strength diagram, where it is not possible to identify any group of sources. The sources in each pulse profile category are very dispersed and no clear groups emerge. Therefore, it does not seem to be a relationship between these parameters and our classification. Nevertheless, this result is interesting in itself due to the fact that it would indicate an independence between the pulse profile and also the accretion column geometry and these two important parameters.

The fact that sources with different pulse profiles (for example IGR J16393-4643, Cen X-3 and GX301-2) have similar values of luminosity and magnetic field strength and with small uncertainties is particularly interesting. In addition, sources with similar profiles (for example Vela X-1 and GX301-2 at energies between 12 and $78 \mathrm{KeV}$ ) have different values of luminosity and magnetic field strength.

However, the case of the source 4U 1901+03 is especially interesting; it was observed in February 2019 during a flare with a variation in its luminosity. This change 

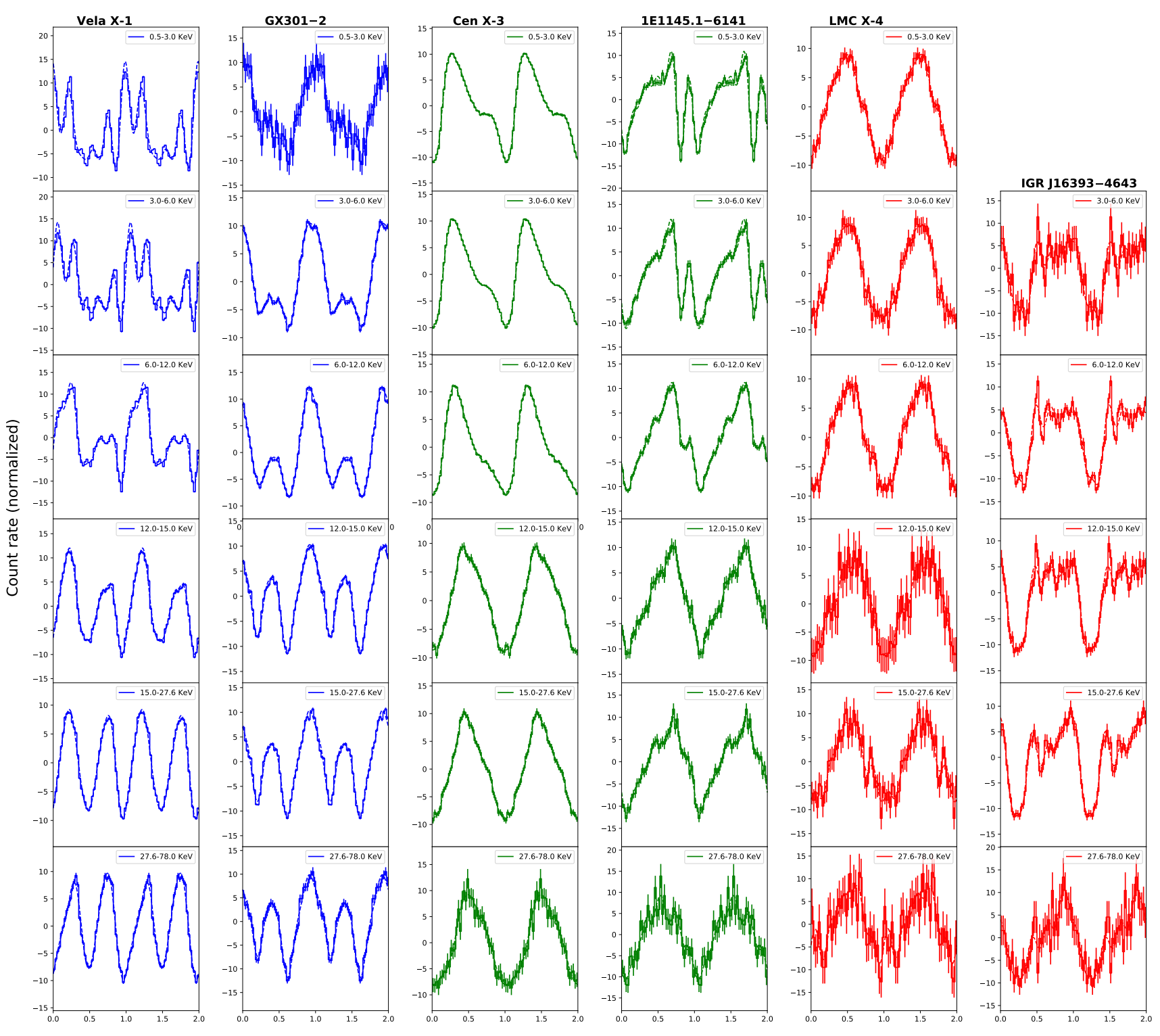

Fig. 3. Comparison of the normalized pulse profiles of different sources of the sample studied at different energy ranges. The sources are in order from left to right: Vela X-1 (type 2), GX $301-2$ (type 2), Cen X-3 (type 1), 1E1145.1-6141 (type 1), LMC X-4 (type 0), and IGR J16393-4643 (type 0). The measured profiles are represented by solid lines, whereas the Fourier fits are represented by dashed lines. The boundaries of the energy ranges are $[0.5,3,6,12,15,27.6$, and 78$] \mathrm{keV}$. The pulse profiles were phase shifted by hand to align the maximums of the pulse profiles of two observations (in cases with observations of both telescopes) and coloured according to their respective pulse profile types in order to better show a comparison between them.

was accompanied by a change in its pulse profile, going from type 1 during the luminosity peak of the flare (the observation with OBS ID $=90502307002$ and a luminosity value of $1.483_{-0.002}^{+0.002} 10^{38} \mathrm{erg} \mathrm{s}^{-1}$ and the observation with OBS ID $=90501305001$ and a luminosity value of $1.242_{-0.005}^{+0.010} 10^{38} \mathrm{erg} \mathrm{s}^{-1}$ ) to type 2 after the luminosity decrease (the observation with OBS ID $=90502307004$ and a luminosity value of $7.87_{-0.03}^{+0.03} \times 10^{37} \mathrm{erg} \mathrm{s}^{-1}$ and the observation with OBS ID $=90501324002$ and a luminosity value of $4.84_{-0.02}^{+0.03} \times$ $10^{37} \mathrm{erg} \mathrm{s}^{-1}$ ).

The pulse profiles are shown in Fig. 6. It is important that the changes in the pulse profiles occurred in all energy ranges, most notably in the lower energy steps. Furthermore, it is especially interesting that the trend of the pulse profile with energy is also modified. Despite showing a change in the pulse profile shape in the whole energy range during the flare, it does not implicate a correlation between the change in the pulse profile and the change in the luminosity. This change could be more related to a modification in the accretion column geometry in addition to a change in disposition of hot material that is around the neutron star during the flare.

The source V $0332+53$ also showed a change in its pulse profile during a flare in 2015. However, in this case some observations had a low signal-to-noise ratio and their pulse profiles were strongly influenced by noise, which means that their types are not well defined. Therefore, the relationship between the pulse profile type and the luminosity is less clear.

Nevertheless, we have presented three of these observations in this paper. The most interesting feature of this case is that the first and the third observation (OBS ID $=80102002002$ and 

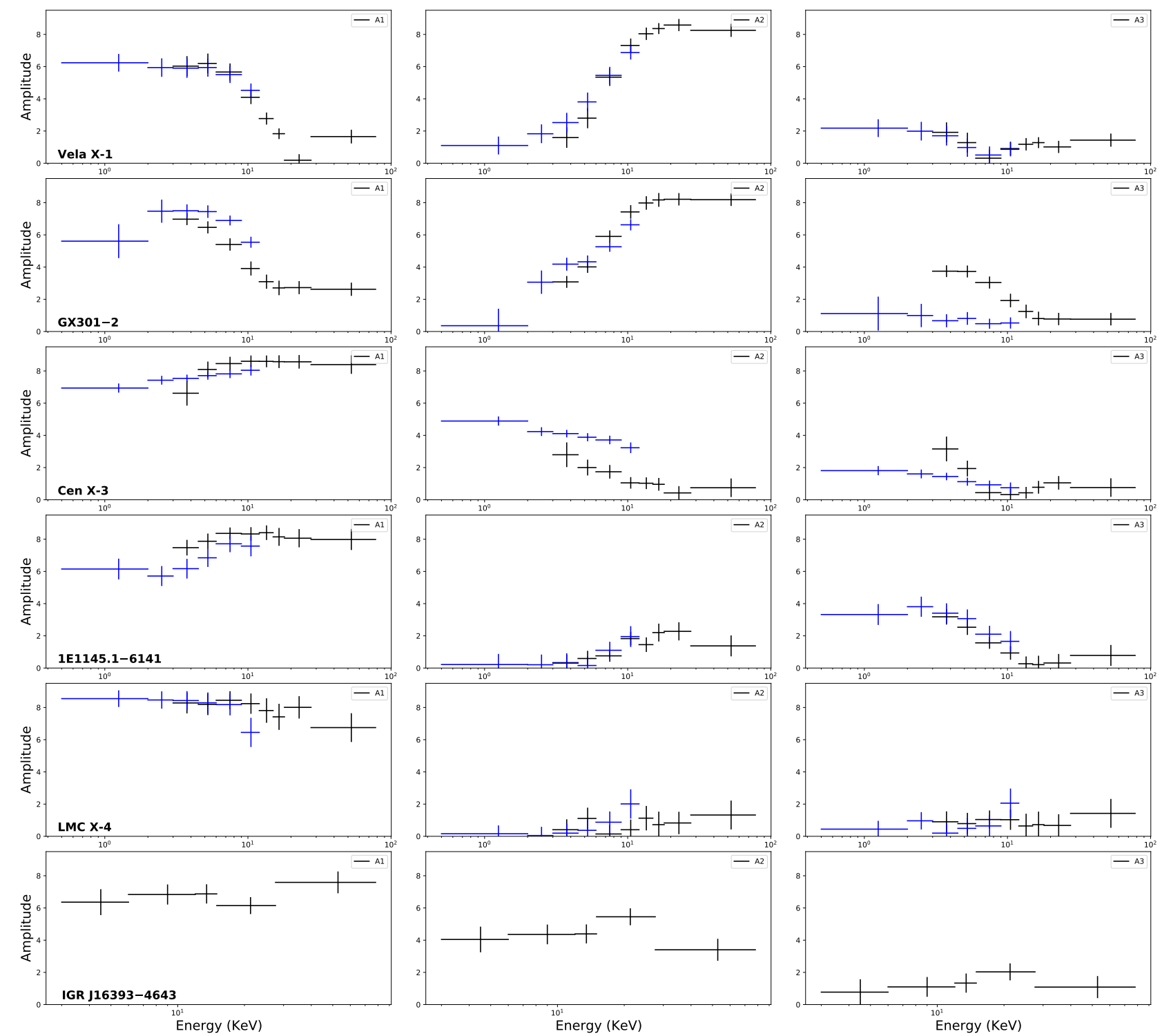

Fig. 4. Comparison of the variation of the first three harmonics (typically those with the largest amplitude) of the Fourier series as a function of energy. The sources are in order from top to bottom: Vela X-1 (type 2), GX 301 - 2 (type 2), Cen X-3 (type 1), 1E1145.1-6141 (type 1), LMC X-4 (type 0), and IGR J16393-4643 (type 0). The boundaries of the energy ranges are [0.5, 2, 3, 4.5, 6, 9, 12, 15, 18, 27.6, and 78] keV with the exception of IGR J16393-4643 whose energy ranges are [3, 6, 12, 15, 27.6, and 78] keV. Blue symbols represent XMM-Newton data and black symbols NUSTAR data.

OBS ID $=80102002010$ ) show two peaks or complex profiles at higher energies, which we have classified as pulse profile type 2 according to the parameters previously explained despite their different luminosity values. They have the same type even though they show clear differences in shape and in the sizes of the peaks, whereas the observation $($ OBS ID $=80102002004)$ with a luminosity value between the other two observations shows only one peak in each energy range, which corresponds to a pulse profile type 0 . An overview of the $\mathrm{V} 0332+53$ energy-resolved pulse profiles is shown in Fig. 7.

It is important to discuss two results from our analysis of $\mathrm{V} 0332+53$. Firstly, our classification system is not perfect; these two pulse profiles classified as type 2 (OBS ID $=80102002002$ and OBS ID $=80102002010$ ) have different pulse profile shapes in some energy ranges, although they have been classified as the same type. However, the goal of the system is to perform systematic classifications based on general parameters and their changes throughout the entire energy range. On the other hand, luminosity is not a determining factor in the characteristics of the pulse profile even in the same source (with the parameters that this involves).

It is likely that other non-observable parameters associated with the accretion rate and the accretion column geometry changed during these flares producing the respective variations in the luminosities and the pulse profiles. This could be due to a common accretion rate factor with energy dependence between the different types, for example a change in the accretion flow that could produce a change in the luminosity and that modifies the incidence of material on the surface of the neutron star.

\subsection{Spin period and orbital parameters}

We also studied the possible correlations between the pulse profile category and the spin and orbital periods, using data shown in Table 2. We plot our results in Fig. 8, which shows the spin period-orbital period, also known as a Corbet diagram 
Table 2. Parameters of the observables of X-ray pulsars sample used in this study, $\left(P_{\text {spin }}\right)$.

\begin{tabular}{|c|c|c|c|c|c|c|c|}
\hline \multirow[t]{2}{*}{ Source } & \multirow{2}{*}{$\begin{array}{c}L_{X} \\
{\left[\operatorname{erg~s}^{-1}\right]}\end{array}$} & \multirow{2}{*}{$\begin{array}{c}B \\
{\left[10^{12} \mathrm{G}\right]}\end{array}$} & \multirow{2}{*}{$\begin{array}{l}P_{\text {orb }} \\
\text { [d] }\end{array}$} & \multicolumn{2}{|c|}{$P_{\text {spin }}{ }^{(*)}$} & \multirow[t]{2}{*}{ Accretion type } & \multirow[t]{2}{*}{ Pulse profile type ${ }^{(*)}$} \\
\hline & & & & $(X M M-N e w t o n)[\mathrm{s}]$ & $($ NuSTAR $)[\mathrm{s}]$ & & \\
\hline SMC X-1 ${ }^{(a)}$ & $(1-50) \times 10^{37}$ & $4.56 \pm 0.20$ & 3.89 & $0.7002 \pm 0.0007$ & $0.70025 \pm 0.00013$ & Accretion disk & 2 \\
\hline \multirow[t]{4}{*}{$4 \mathrm{U} 1901+03^{(b)}$} & $4.84_{-0.02}^{+0.03} \times 10^{37}$ & $0.4 \pm 0.1$ & 22.5 & & $2.7608 \pm 0.0008$ & BeXRB & 2 \\
\hline & $1.483_{-0.002}^{+0.002} \times 10^{38}$ & - & - & & $2.7616 \pm 0.0008$ & - & 1 \\
\hline & $7.87_{-0.03}^{+0.002} \times 10^{37}$ & - & - & & $2.7622 \pm 0.0008$ & - & 2 \\
\hline & $1.242_{-0.005}^{+0.000} \times 10^{38}$ & - & - & & $2.7629 \pm 0.0008$ & - & 1 \\
\hline \multirow[t]{3}{*}{$\mathrm{V} 0332+53^{(c)}$} & $(1.24-1.74) \times 10^{38}$ & $2.44 \pm 0.05$ & 33.9 & & $4.3759 \pm 0.0015$ & BeXRB & 2 \\
\hline & $(5.7-9.5) \times 10^{37}$ & - & - & & $4.3759 \pm 0.0017$ & - & 0 \\
\hline & $(5.3-14) \times 10^{36}$ & - & - & & $4.3767 \pm 0.0016$ & - & 2 \\
\hline Cen X-3 ${ }^{(d)}$ & $(1-5) \times 10^{37}$ & $2.9-3.2$ & 2.09 & $4.802 \pm 0.005$ & $4.797 \pm 0.003$ & Accretion disk & 1 \\
\hline LMC X-4 ${ }^{(e)}$ & $(2-5) \times 10^{38}$ & $10-30$ & 1.41 & $13.48 \pm 0.05$ & $13.49 \pm 0.03$ & Accretion disk & 0 \\
\hline GRO J2058+42 $(f)$ & $(4.0-4.4) \times 10^{37}$ & $1.1-1.6$ & $53.9 \pm 1.2 / 110 \pm 3$ & & $194 \pm 3$ & BeXRB & 2 \\
\hline Vela X-1 ${ }^{(g)}$ & $(1-10) \times 10^{36}$ & $2.60 \pm 0.01$ & 8.96 & $283 \pm 4$ & $283 \pm 6$ & Wind-fed & 2 \\
\hline 1E1145.1-6141 ${ }^{(h)}$ & $(1-10) \times 10^{36}$ & & 14.4 & $297 \pm 6$ & $297 \pm 8$ & Wind-fed & 1 \\
\hline IGR J17252-3616 ${ }^{(i)}$ & $(6-16) \times 10^{35}$ & $0.71 \pm 0.03$ & 9.74 & $412 \pm 15$ & & Wind-fed & 0 \\
\hline $\mathrm{GX} 301-2^{(j)}$ & $(8-20) \times 10^{36}$ & $3.89 \pm 0.43$ & 41.5 & $680 \pm 30$ & $690 \pm 20$ & Wind-fed & 2 \\
\hline IGR J16393-4643 ${ }^{(k)}$ & $(5-20) \times 10^{36}$ & $2.5 \pm 0.1$ & 4.24 & & $900 \pm 20$ & Wind-fed & 0 \\
\hline
\end{tabular}

Notes. Column (1): name of the observed source, Col. (2): x-ray luminosity, Col. (3): magnetic field strength, Cols. (4) and (5): spin period obtained from XMM-Newton and NUSTAR observations respectively, Col. (6): type of accretion mechanism, and Col. (7): pulse profile type according to the classification obtained in this study. The sources are ordered by their spin periods. (*) The $P_{\text {spin }}$ values were obtained with the epoch folding statistic and their uncertainties as a FWHM/2 of the fitted Lorentzian distribution for each observation. The pulse profile types were obtained by qualitative classification in the groups described in this section.

References. ${ }^{(a)}$ Ephemeris from Falanga et al. (2015); Brumback et al. (2020); other values from Pike et al. (2019); Pradhan et al. (2020). ${ }^{(b)}$ Ephemeris from Sootome et al. (2011); luminosities from Coley et al. (priv. comm.); magnetic field from Reig \& Milonaki (2016). ${ }^{(c)}$ Ephemeris from Doroshenko et al. (2016); other values from Vybornov et al. (2018). ${ }^{(d)}$ Ephemeris from M.H.Finger (HEAD 2010 poster); other values from Shtykovsky et al. (2017). ${ }^{(e)}$ Ephemeris from Levine et al. (2000); other values from Shtykovsky et al. (2017). ${ }^{(f)}$ Ephemeris not determined; other values from Mukerjee et al. (2020); Corbet et al. (1997); Wilson et al. (1998). ${ }^{(g)}$ Ephemeris from Kreykenbohm et al. (2008); other values from Sidoli et al. (2015); Kretschmar et al. (2019b). ${ }^{(h)}$ Ephemeris from Ray \& Chakrabarty (2002); other values from Ferrigno et al. (2007). ${ }^{(i)}$ Ephemeris from Manousakis \& Walter (2010); other values from Manousakis et al. (2012); Zurita Heras et al. (2006). ${ }^{(j)}$ Ephemeris from Doroshenko et al. (2010); other values from Nishimura (2014). ${ }^{(k)}$ Ephemeris not determined; other values from Bodaghee et al. (2016); Islam et al. (2015).

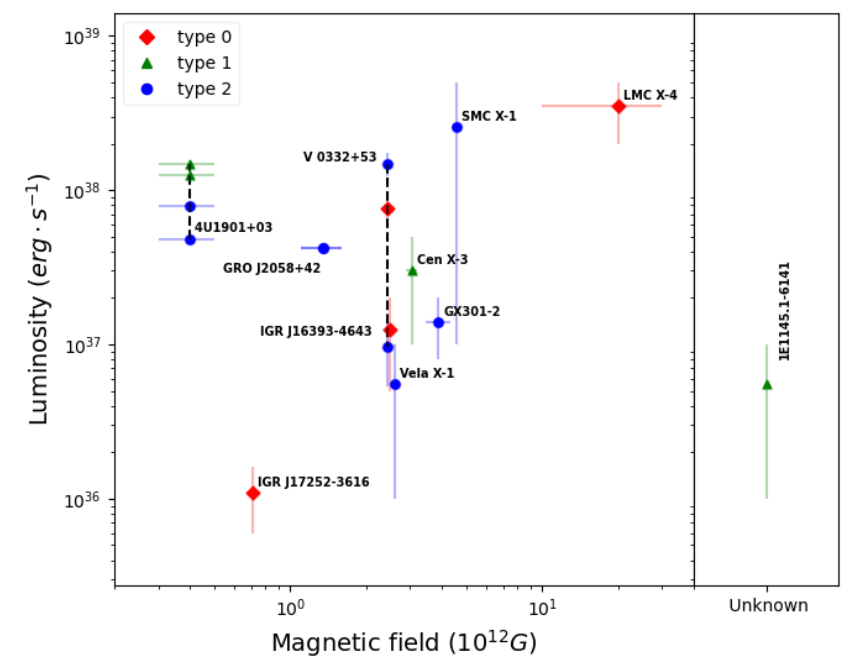

Fig. 5. Luminosity $\left(L_{X}\right)$ vs. magnetic field strength $(B)$ diagram. The shape and colour of the symbols indicate the different pulse profile groups.

(Corbet 1986). In this diagram the different classes of HMXBs are separated into three distinct groups according to their accretion mechanism: stellar wind fed, Roche lobe overflow, and BeXRBs.

Although the sample of sources is small, the pulse profile types are distributed over different accretion mechanisms and no clear relation to the rotation and spin periods is obvious. However, this indicates that the accretion mechanism does not have a decisive role in the shape of the accretion column and emission pattern.

However, there is an indication that type 0 sources cluster in the upper left part of the diagram (except for $\mathrm{V} \mathrm{0332+53}$ (OBS ID = 80102002004) even though this source presents a low $\mathrm{S} / \mathrm{N}$ and its type is not well-defined) with relatively fast orbital periods and relatively slow spin periods. This distribution could indicate that these sources are somewhat older and have a simpler magnetic field geometry leading to a more symmetric pulse profile. However, with only three sources any such interpretation is currently speculation and would need to be supported by further data from more sources.

The pulse profiles will also depend on the inclination angle of the neutron star spin axis to our line of sight. However, there is no known method to directly measure this angle, or even the inclination of the orbital plane of the binary system Tomsick \& Muterspaugh (2010). We therefore cannot investigate this dependence currently.

\section{Conclusions}

In this paper we have presented a framework used to study and classify energy-resolved pulse profiles based on a decomposition into their Fourier components. We have applied this framework to a small sample of X-ray pulsars, for which a wide energy coverage between XMM-Newton and NUSTAR is available. The 

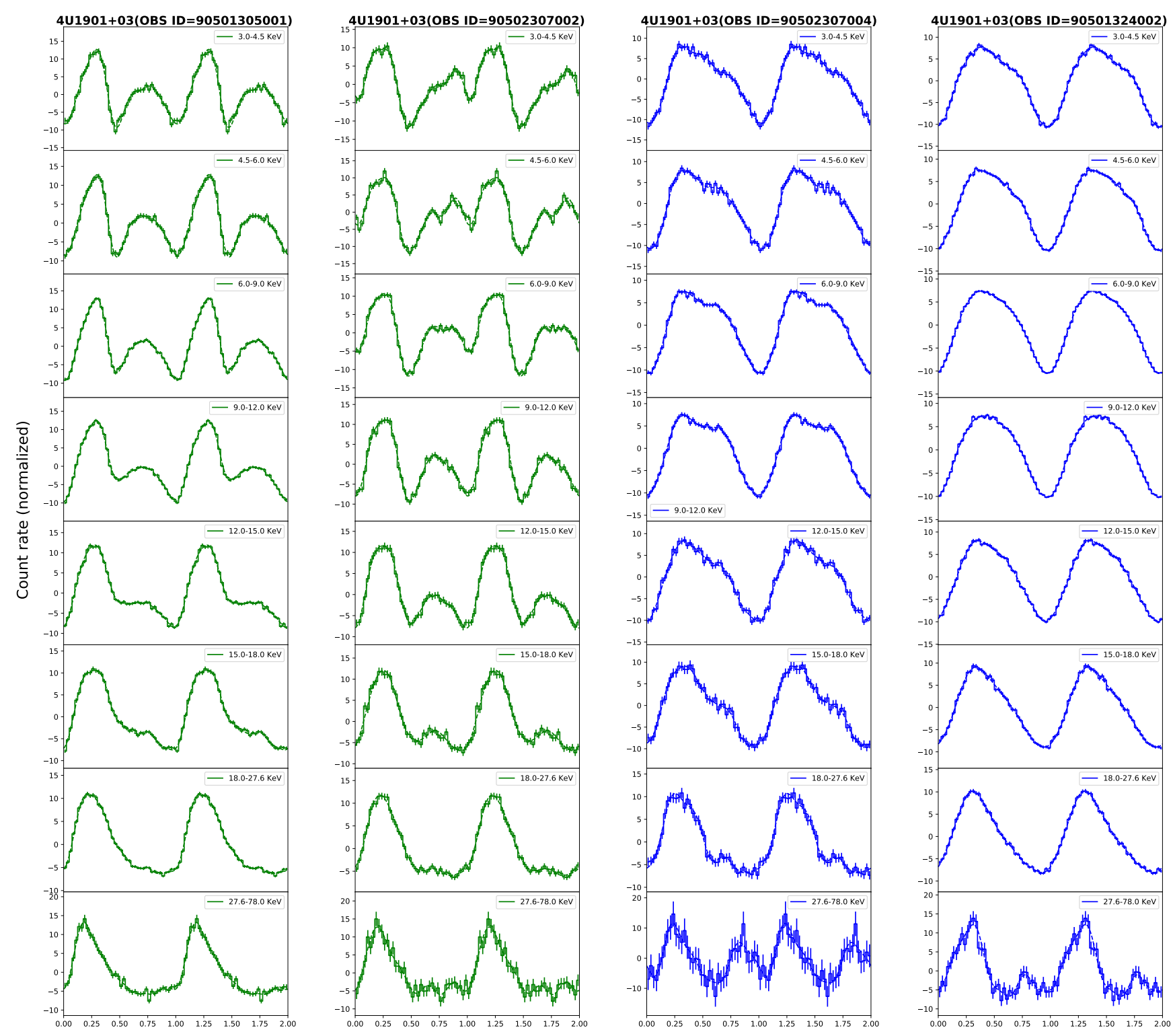

Fig. 6. Comparison of normalized pulse profiles of source 4 U 1901+03 studied at different energy ranges in different stages during a flare period. The observations are in temporal order from left to right: observation with OBS ID $=90501305001$ and a luminosity value of $1.242_{-0.005}^{+0.010} \times$ $10^{38} \mathrm{erg} \mathrm{s}^{-1}$ with a pulse profile type 1 ; observation with OBS ID $=90502307002$ and a luminosity value of $1.483_{-0.002}^{+0.002} \times 10^{38} \mathrm{erg} \mathrm{s}^{-1}$ with a pulse profile type 1; observation with OBS ID $=90502307004$ and a luminosity value of $7.87_{-0.03}^{+0.03} \times 10^{37} \mathrm{erg} \mathrm{s}^{-1}$ with a pulse profile type 2 ; observation with OBS ID $=90501324002$ and a luminosity value of $4.84_{-0.02}^{+0.03} \times 10^{37} \mathrm{erg} \mathrm{s}^{-1}$ with a pulse profile type 2 . The measured profiles are represented by solid lines, whereas the Fourier fits are represented by dashed lines. The boundaries of the energy ranges are [0.5, 3, 6, 12, 15, 27.6, and 78] keV. The pulse profiles were shifted by hand to align the minima of the pulse profiles and coloured according to their respective pulse profile types in order to better show a comparison between them.

framework is publicly available and can be used to create energy-resolved and decomposed pulse profiles for any event data.

In our sample we find a range of diverse shapes and energy variation of the pulse profiles. Based on the results of the amplitudes and phases of the Fourier components we identified three distinct groups. We investigated correlations between these groups and other physical parameters of the sources, such as luminosity, magnetic field strength, spin period, and orbital period. However, we could not identify any clear connections.

We only find an interesting, but not yet statistically significant connection, for profile type 0 , which seems to be prevalent for sources with a combination of relatively short orbital periods and slow spin periods (Fig. 8). Type 0 sources are characterized by little to no energy dependence of their pulse profiles, indicating that there is no energy dependence in the emission region or geometry. How this could be related to spin or orbital periods is, however, beyond the scope of this paper.

In 4 U 1901+03 we find that the source changes from profile type 1 to 2 with decreasing luminosity during the outburst. This clearly indicates a significant change in the accretion column and emission geometry as a function of accretion rate and luminosity. Becker et al. (2012) propose that such a change could be related to the accretion column switching from a Coulomb dominated deceleration regime to a radiation dominated region (i.e. the source becoming super-critical at high luminosities). 

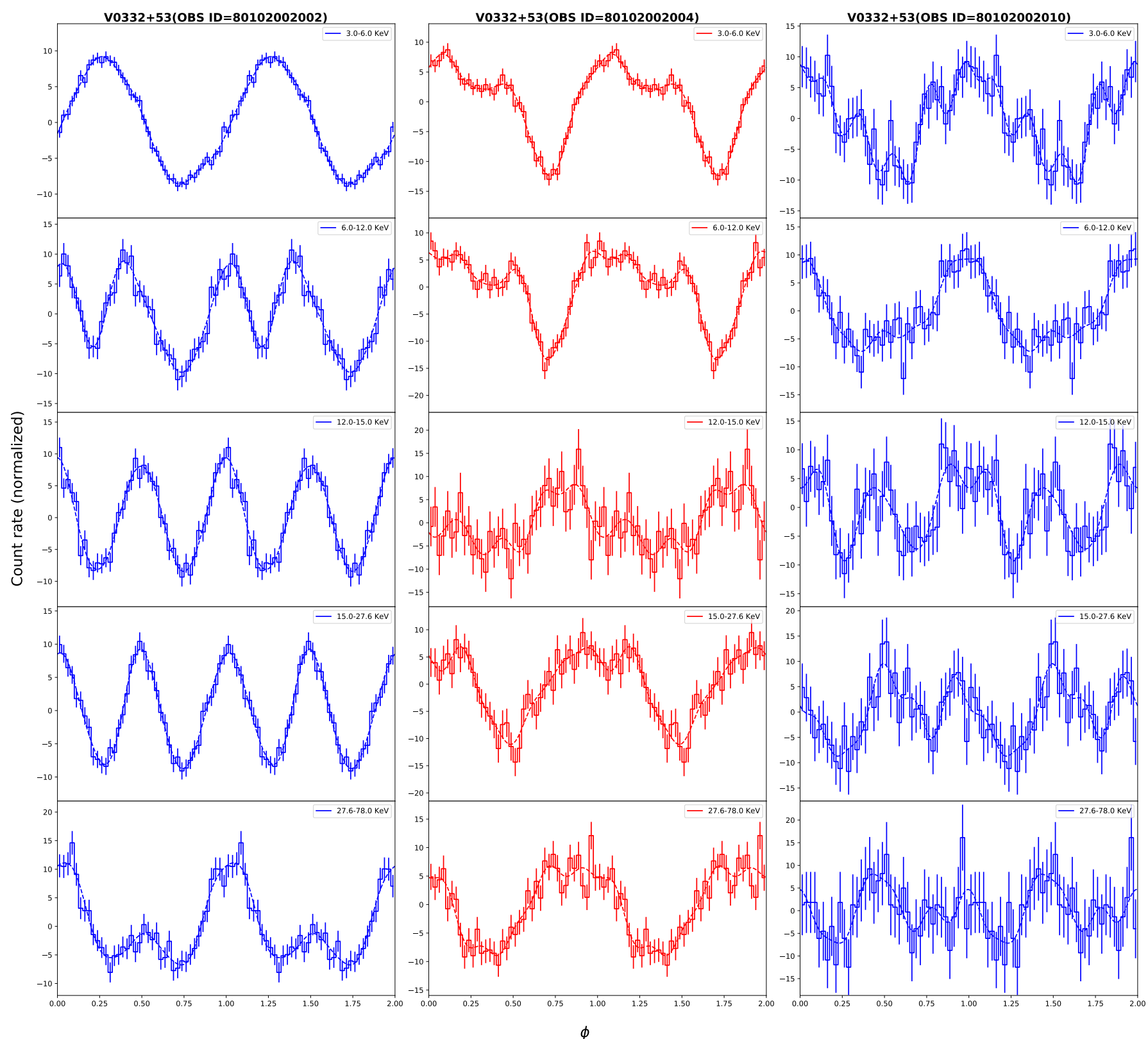

Fig. 7. Comparison of normalized pulse profiles of source V $0332+53$ studied at different energy ranges in different stages during a flare period. The observations are in temporal order from left to right: observation with OBS ID $=80102002002$ and a luminosity value of $(1.24-1.74) \times 10^{38} \mathrm{erg} \mathrm{s}^{-1}$ with a pulse profile type 2 ; observation with OBS ID $=80102002004$ and a luminosity value of $(5.7-9.5) \times 10^{37} \mathrm{erg} \mathrm{s}^{-1}$ with a pulse profile type 0 ; observation with OBS ID $=80102002010$ and a luminosity value of $(5.3-14) \times 10^{36} \mathrm{erg} \mathrm{s}^{-1}$ with a pulse profile type 2 . The measured profiles are represented by solid lines, whereas the Fourier fits are represented by dashed lines. The boundaries of the energy ranges are [0.5, 3, 6, 12, 15, 27.6, and $78 \mathrm{keV}$. The pulse profiles were shifted by hand to align the minima of the pulse profiles and coloured according to their respective pulse profile types in order to better show a comparison between them.

With the formation of a radiation dominated shock, we expect a significant change in the emission geometry, as observed here.

However, a more quantitative comparison between expected pulse profiles as a function of accretion regime and the data is very complex. This complexity is mainly due to the many different physical effects involved, on the micro and the macro scale. Small changes in emission height or in-falling velocity can have large effects on the observed pulse profile due to the strong light bending around the neutron star (cf. Falkner 2018).

Our framework provides a simple and straightforward way to decompose pulse profiles into their fundamental components, which will help further theoretical efforts to model the profiles based on first principle physics. By identifying the dominant harmonics and contributions in the pulse profiles, we provide information for the models where most of the energy-dependent changes occur.

By increasing the sample size with new and archival observations of accretion pulsars in the future, we can populate Figs. 5 and 8 further and investigate in more detail the correlation with physical parameters. With more data it will also be possible to refine the definition of the pulse profile types and identify a more general classification scheme. We believe that our proposed classification can be a useful tool in understanding and sorting X-ray 


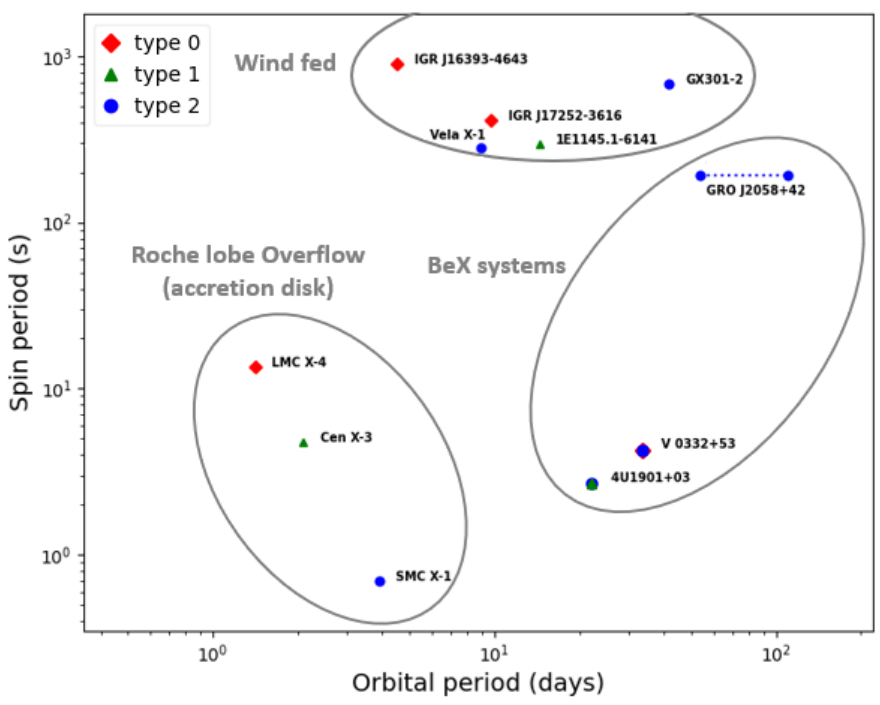

Fig. 8. Spin period vs orbital period for the source sample. The shape and colour of the symbols indicate the different pulse profile groups and the grey lines indicate the regions of the different types of HMXBs by their accretion method.

pulsars and in looking for common physical properties within the accretion column.

Acknowledgements. This research has made use of data and software provided by the High Energy Astrophysics Science Archive Research Center (HEASARC), which is a service of the Astrophysics Science Division at NASA/GSFC as well as NASA'sAstrophysics Data System Bibliographic Service (ADS). This work made use of data from the NuSTAR mission, a project led by the California Institute of Technology, managed by the Jet Propulsion Laboratory, and funded by the National Aeronautics and Space Administration and used the NuSTAR Data Analysis Software (NuSTARDAS) jointly developed by the ASI Science Data Center (ASDC, Italy) and the California Institute of Technology (USA). This research has also made use of data obtained with XMM-Newton, an ESA science mission with instruments and contributions directly funded by ESA Member States. A.M.J. acknowledges partial funding from the European Space Agency (ESA) under partnership agreement 4000133194/20/NL/MH/hm between ESA and FAU Erlangen-Nürnberg.

\section{References}

Astropy Collaboration (Robitaille, T. P., et al.) 2013, A\&A, 558, A33

Basko, M. M., \& Sunyaev, R. A. 1975, A\&A, 42, 311

Becker, P. A., \& Wolff, M. T. 2005, ApJ, 630, 465

Becker, P. A., \& Wolff, M. T. 2007, ApJ, 654, 435

Becker, P. A., Klochkov, D., Schönherr, G., et al. 2012, A\&A, 544, A123

Beloborodov, A. M. 2002, ApJ, 566, L85

Bodaghee, A., Tomsick, J. A., Fornasini, F. M., et al. 2016, ApJ, 823, 146

Brumback, M. C., Hickox, R. C., Fürst, F. S., et al. 2020, ApJ, 888, 125

Brumback, M. C., Hickox, R. C., Fürst, F. S., et al. 2021, ApJ, 909, 186

Bulik, T., Riffert, H., Meszaros, P., et al. 1995, ApJ, 444, 405

Caballero, I., Kraus, U., Santangelo, A., Sasaki, M., \& Kretschmar, P. 2011 A\&A, 526, A131

Camero Arranz, A., Wilson, C. A., Finger, M. H., \& Reglero, V. 2007, A\&A, 473,551

Campana, S., \& Di Salvo, T. 2018, The Physics and Astrophysics of Neutron Stars, Accreting Pulsars: Mixing-up Accretion Phases in Transitional Systems, eds. L. Rezzolla, P. Pizzochero, D. I. Jones, N. Rea, \& I. Vidaña (Berlin: Springer), 457, 149
Cappallo, R., Laycock, S. G. T., Christodoulou, D. M., Coe, M. J., \& Zezas, A. 2019, MNRAS, 486, 3248

Corbet, R. H. D. 1986, MNRAS, 220, 1047

Corbet, R., Peele, A., \& Remillard, R. 1997, IAU Circ., 6556, 3

Doroshenko, V., Santangelo, A., Suleimanov, V., et al. 2010, A\&A, 515, A10

Doroshenko, V., Tsygankov, S., \& Santangelo, A. 2016, A\&A, 589, A72

El Mellah, I., Sander, A. A. C., Sundqvist, J. O., \& Keppens, R. 2019, A\&A, 622 A189

Falanga, M., Bozzo, E., Lutovinov, A., et al. 2015, A\&A, 577, A130

Falkner, S. 2018, PhD thesis, Universität Erlangen-Nürnberg, Germany

Ferrigno, C., Segreto, A., Santangelo, A., et al. 2007, A\&A, 462, 995

Finger, M. H., Bildsten, L., Chakrabarty, D., et al. 1999, ApJ, 517, 449

Harrison, F. A., Craig, W. W., Christensen, F. E., et al. 2013, ApJ, 770, 103

Hickox, R. C., \& Vrtilek, S. D. 2005, ApJ, 633, 1064

Hickox, R. C. Narayan, R., \& Kallman, T. R. 2004, ApJ, 614, 881

Huppenkothen, D., Bachetti, M., Stevens, A. L., et al. 2019, ApJ, 881, 39

Islam, N., Maitra, C., Pradhan, P., \& Paul, B. 2015, MNRAS, 446, 4148

Iwakiri, W. B., Pottschmidt, K., Falkner, S., et al. 2019, ApJ, 878, 121

Jansen, F., Lumb, D., Altieri, B., et al. 2001, A\&A, 365, L1

Kanno, S. 1980, PASJ, 32, 105

Kraus, U., Nollert, H.-P., Ruder, H., \& Riffert, H. 1995, ApJ, 450, 763

Kretschmar, P., Fürst, F., Sidoli, L., et al. 2019a, New Astron. Rev., 86, 101546

Kretschmar, P., Martínez-Núñez, S., Fürst, F., et al. 2019b, Mem. Soc. Astron. It., 90, 221

Kretschmar, P., El Mellah, I., Martínez-Núñez, S., et al. 2021, A\&A, 652 A95

Kreykenbohm, I., Wilms, J., Kretschmar, P., et al. 2008, A\&A, 492, 511

Leahy, D. A. 1991, MNRAS, 251, 203

Leahy, D. A. 2004, MNRAS, 348, 932

Leahy, D. A., Darbro, W., Elsner, R. F., et al. 1983, ApJ, 266, 160

Levine, A. M., Rappaport, S. A., \& Zojcheski, G. 2000, ApJ, 541, 194

Liu, Q. Z., van Paradijs, J., \& van den Heuvel, E. P. J. 2006, A\&A, 455, 1165

Liu, Q. Z., van Paradijs, J., \& van den Heuvel, E. P. J. 2007, A\&A, 469, 807

Manousakis, A., \& Walter, R. 2010, PoS, INTEGRAL2010, 066

Manousakis, A., Walter, R., \& Blondin, J. M. 2012, A\&A, 547, A20

Martínez-Núñez, S., Kretschmar, P., Bozzo, E., et al. 2017, Space Sci. Rev., 212 59

Mukerjee, K., Antia, H. M., \& Katoch, T. 2020, ApJ, 897, 73

Negueruela, I., \& Okazaki, A. T. 2001, A\&A, 369, 108

Nishimura, O. 2014, ApJ, 781, 30

Nixon, C. J., \& Pringle, J. E. 2020, ApJ, 905, L29

Okazaki, A. T., \& Negueruela, I. 2001, A\&A, 377, 161

Pike, S. N., Harrison, F. A., Bachetti, M., et al. 2019, ApJ, 875, 144

Poutanen, J., \& Beloborodov, A. M. 2006, MNRAS, 373, 836

Poutanen, J., Ibragimov, A., \& Annala, M. 2009, ApJ, 706, L129

Pradhan, P., Maitra, C., \& Paul, B. 2020, ApJ, 895, 10

Pringle, J. E., \& Rees, M. J. 1972, A\&A, 21, 1

Ray, P. S., \& Chakrabarty, D. 2002, ApJ, 581, 1293

Reig, P. 2011, Ap\&SS, 332, 1

Reig, P., \& Milonaki, F. 2016, A\&A, 594, A45

Riffert, H., \& Meszaros, P. 1988, ApJ, 325, 207

Riffert, H., Nollert, H. P., Kraus, U., \& Ruder, H. 1993, ApJ, 406, 185

Riley, T. E., Watts, A. L., Bogdanov, S., et al. 2019, ApJ, 887, L21

Sasaki, M., Müller, D., Kraus, U., Ferrigno, C., \& Santangelo, A. 2012, A\&A, 540, A35

Shtykovsky, A. E., Lutovinov, A. A., Arefiev, V. A., et al. 2017, Astron. Lett., 43, 175

Sidoli, L., Paizis, A., Fürst, F., et al. 2015, MNRAS, 447, 1299

Sootome, T., Nakajima, M., Mihara, T., et al. 2011, ATel, 3829, 1

Strüder, L., Briel, U., Dennerl, K., et al. 2001, A\&A, 365, L18

Tomsick, J. A., \& Muterspaugh, M. W. 2010, ApJ, 719, 958

Vybornov, V., Doroshenko, V., Staubert, R., \& Santangelo, A. 2018, A\&A, 610, A88

Wang, Y. M., \& Welter, G. L. 1981, A\&A, 102, 97

Wilson, C. A., Finger, M. H., Harmon, B. A., Chakrabarty, D., \& Strohmayer, T. 1998, ApJ, 499, 820

Wolff, M. T., Becker, P. A., Gottlieb, A. M., et al. 2016, ApJ, 831, 194

Zurita Heras, J. A., De Cesare, G., Walter, R., et al. 2006, A\&A, 448, 261 


\section{Appendix A: Pulse profiles and harmonic amplitudes}
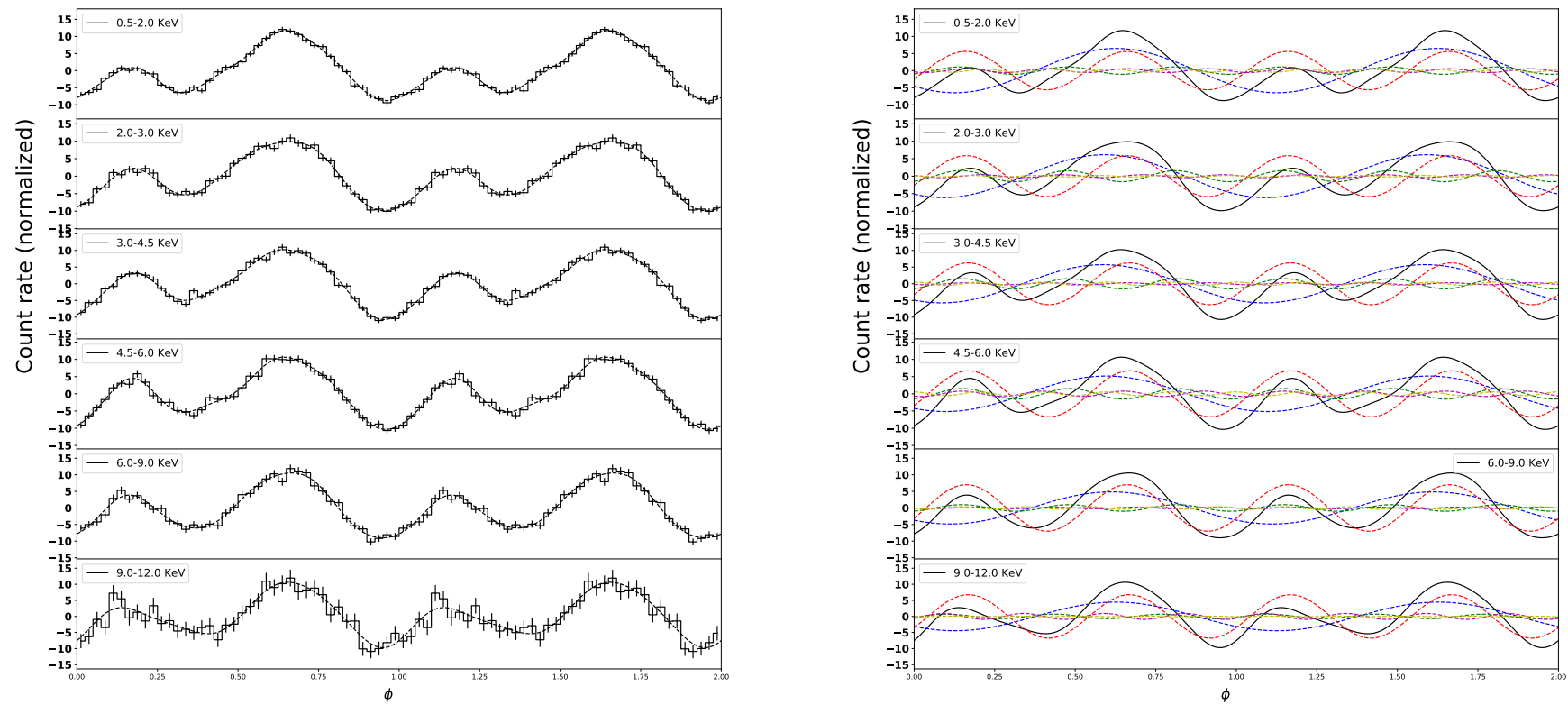

Fig. A.1. Comparison of the observed energy resolved pulse profiles of SMC X-1 and their Fourier fits at different energy bands. Left: Normalized pulse profiles of SMC X-1 at different energy ranges based on XMM-Newton data. The measured profiles are represented by solid lines, whereas the Fourier fits are represented by dashed lines. The boundaries of the energy ranges are $[0.5,2,3,4.5,6,9,12]$ keV. Right: Overall approximate pulse profile (black solid line) and Fourier components 1-5 (coloured dashed lines: blue, red, green, purple, and yellow, respectively).
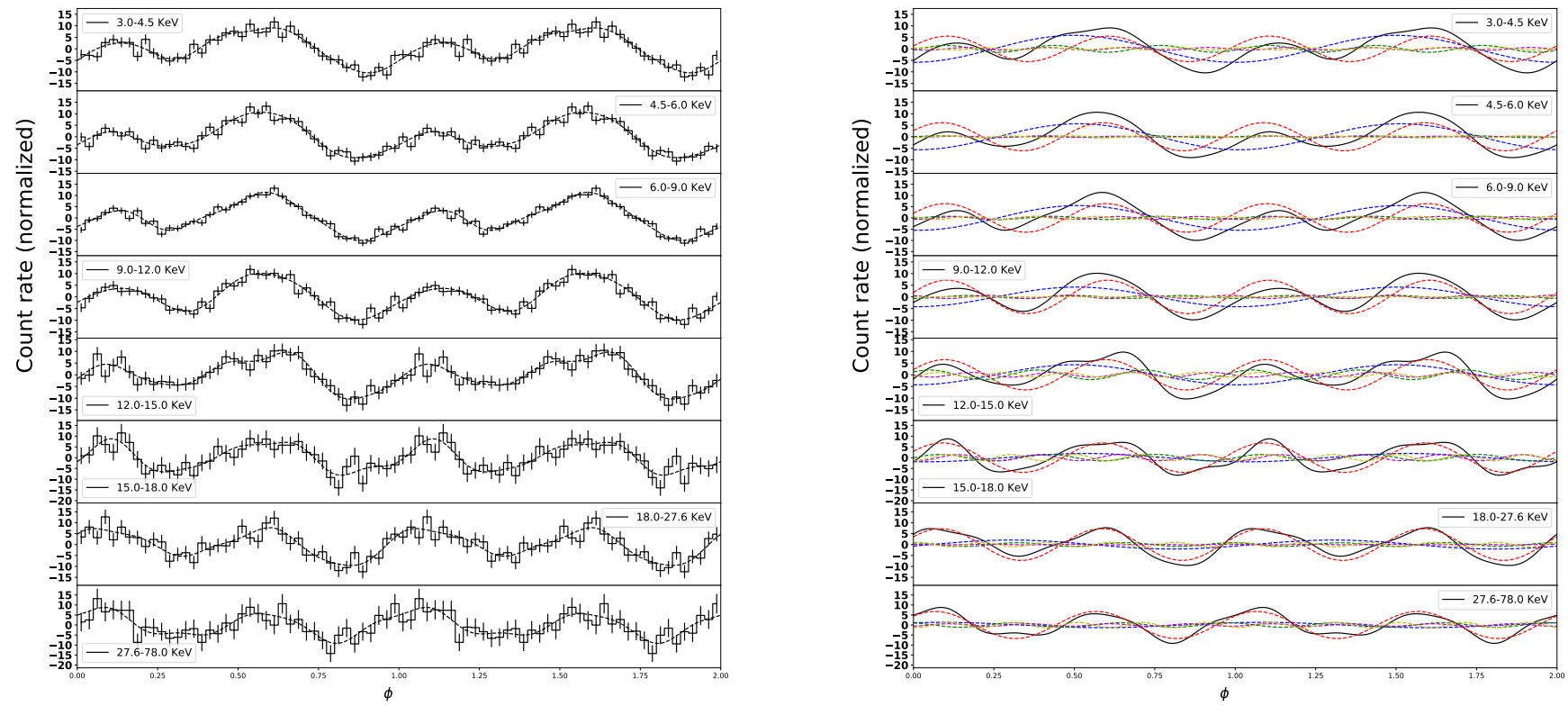

Fig. A.2. Comparison of the observed energy resolved pulse profiles of SMC X-1 and their Fourier fits at different energy bands. Left: Normalized pulse profiles of SMC X-1 at different energy ranges based on NuSTAR data. The measured profiles are represented by solid lines, whereas the Fourier fits are represented by dashed lines. The boundaries of the energy ranges are [3, 4.5, 6, 9, 12, 15, 18, 27.6, and 78] keV. Right: Overall approximate pulse profile (black solid line) and Fourier components 1-5 (coloured dashed lines: blue, red, green, purple, and yellow, respectively). 
J. Alonso-Hernández et al.: Common patterns in pulse profiles of HMXBs
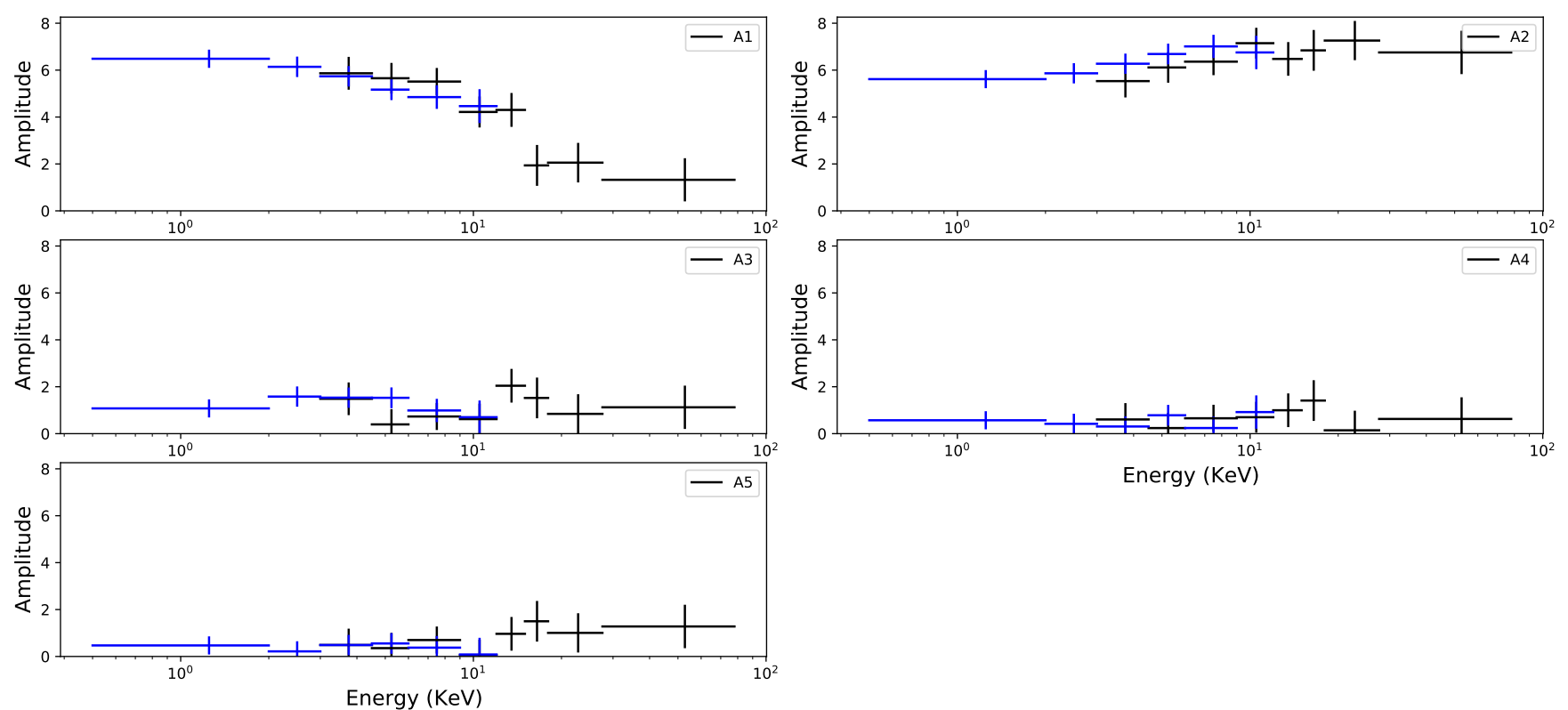

Energy $(\mathrm{KeV})$

Fig. A.3. Variation of the harmonic amplitudes for SMC X-1. The boundaries of the energy ranges are $[0.5,2,3,4.5,6,9,12,15,18,27.6$, and $78] \mathrm{keV}$. The XMM-Newton data is represented by blue markers and NUSTAR data is represented by black markers.
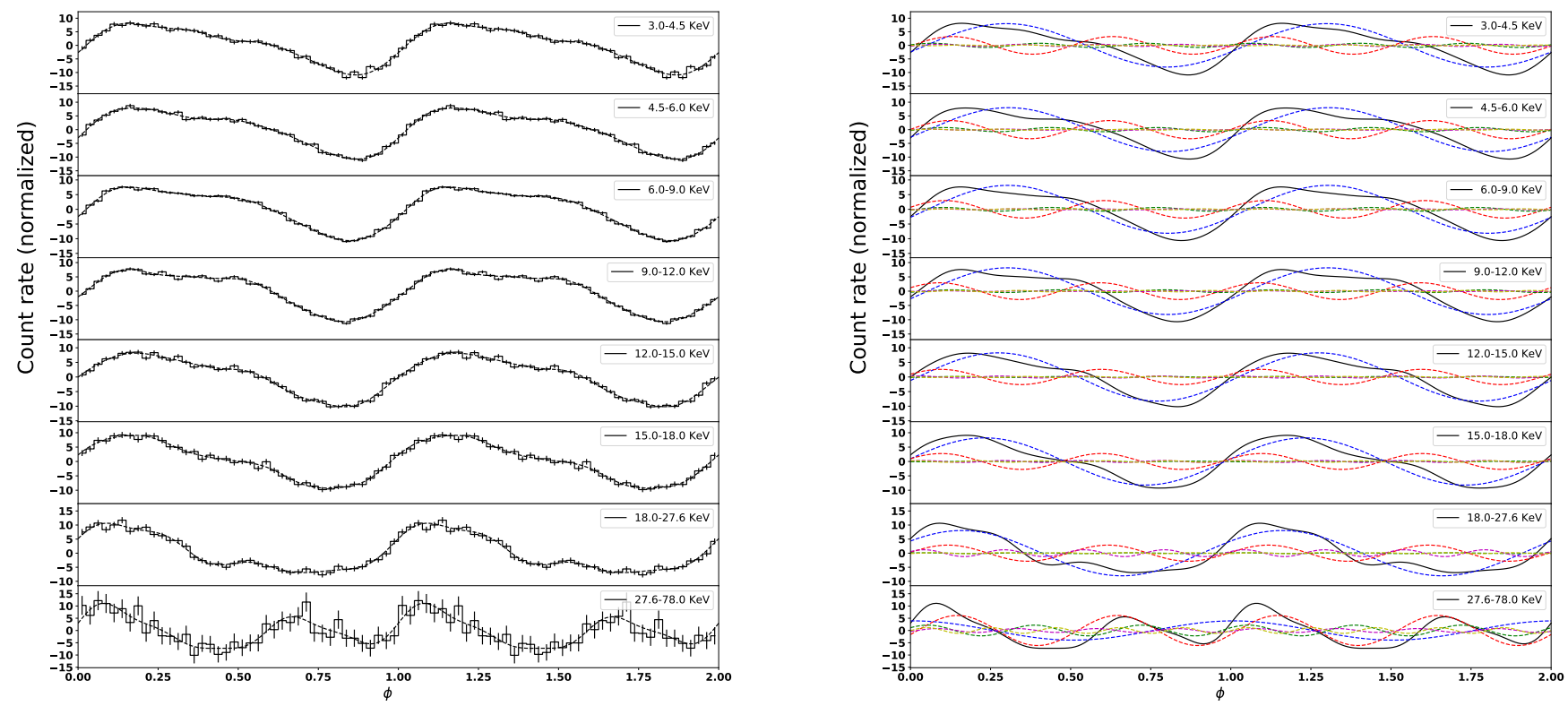

Fig. A.4. Comparison of the observed energy resolved pulse profiles of 4 U 1901+03 (observation with OBS ID=90502307004 and a luminosity value of $7.87_{-0.03}^{+0.03} \cdot 10^{37} \mathrm{erg} \cdot \mathrm{s}^{-1}$ ) and their Fourier fits at different energy bands. Left: Normalized pulse profiles of 4U 1901+03 (observation with OBS ID=90502307004 and a luminosity value of $7.87_{-0.03}^{+0.03} \cdot 10^{37} \mathrm{erg} \cdot \mathrm{s}^{-1}$ ) at different energy ranges based on NuSTAR data. The measured profiles are represented by solid lines, whereas the Fourier fits are represented by dashed lines. The boundaries of the energy ranges are [3, 4.5, 6, 9, 12, 15, 18, 27.6, and 78] keV. Right: Overall approximate pulse profile (black solid line) and Fourier components 1-5 (coloured dashed lines: blue, red, green, purple, and yellow, respectively). 

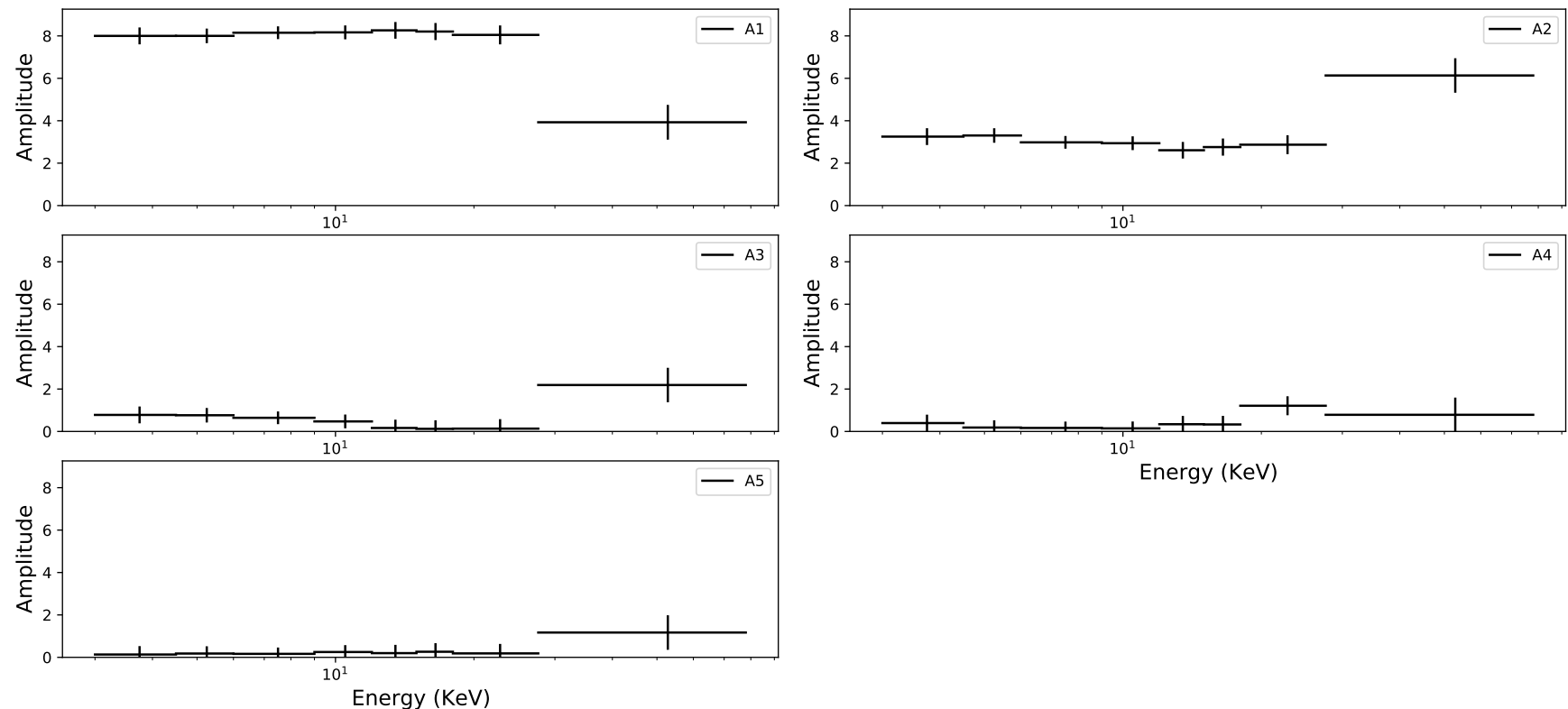

Energy $(\mathrm{KeV})$

Fig. A.5. Variation of the harmonic amplitudes for $4 \mathrm{U} 1901+03$ (observation with OBS ID=90502307004 and a luminosity value of $7.87_{-0.03}^{+0.03}$. $\left.10^{37} \mathrm{erg} \cdot \mathrm{s}^{-1}\right)$. The boundaries of the energy ranges are $[3,4.5,6,9,12,15,18,27.6$, and 78] keV. Only the NuSTAR data are represented by black markers.
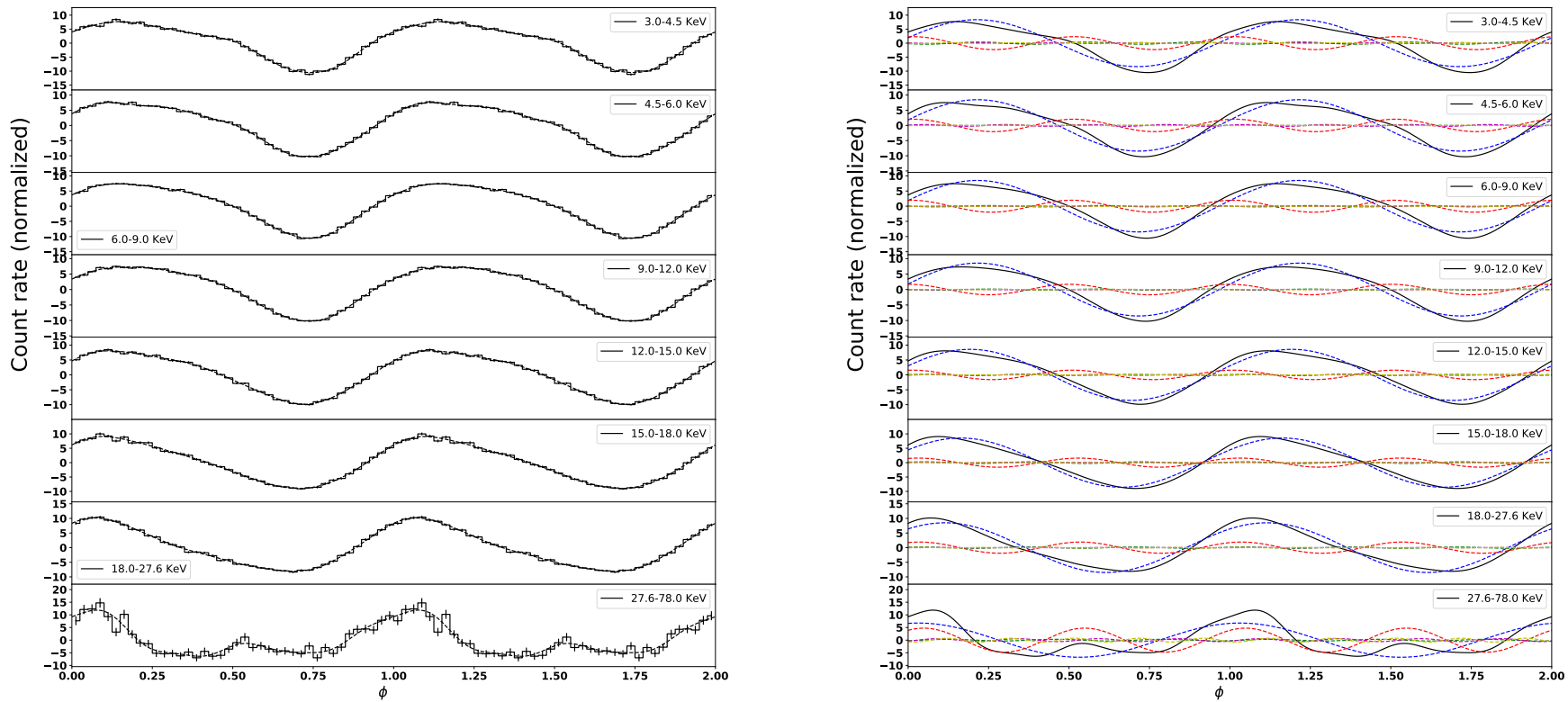

Fig. A.6. Comparison of the observed energy resolved pulse profiles of 4 U 1901+03 (observation with OBS ID= 90501324002 and a luminosity value of $4.84_{-0.02}^{+0.03} \cdot 10^{37} \mathrm{erg} \cdot \mathrm{s}^{-1}$ ) and their Fourier fits at different energy bands. Left: Normalized pulse profiles of 4U 1901+03 (observation with OBS ID $=90501324002$ and a luminosity value of $4.84_{-0.02}^{+0.03} \cdot 10^{37} \mathrm{erg} \cdot \mathrm{s}^{-1}$ ) at different energy ranges based on NuSTAR data. The measured profiles are represented by solid lines, whereas the Fourier fits are represented by dashed lines. The boundaries of the energy ranges are [3, 4.5, 6, 9, 12, 15, 18, 27.6, and 78] keV. Right: Overall approximate pulse profile (black solid line) and Fourier components 1-5 (coloured dashed lines: blue, red, green, purple, and yellow, respectively). 
J. Alonso-Hernández et al.: Common patterns in pulse profiles of HMXBs
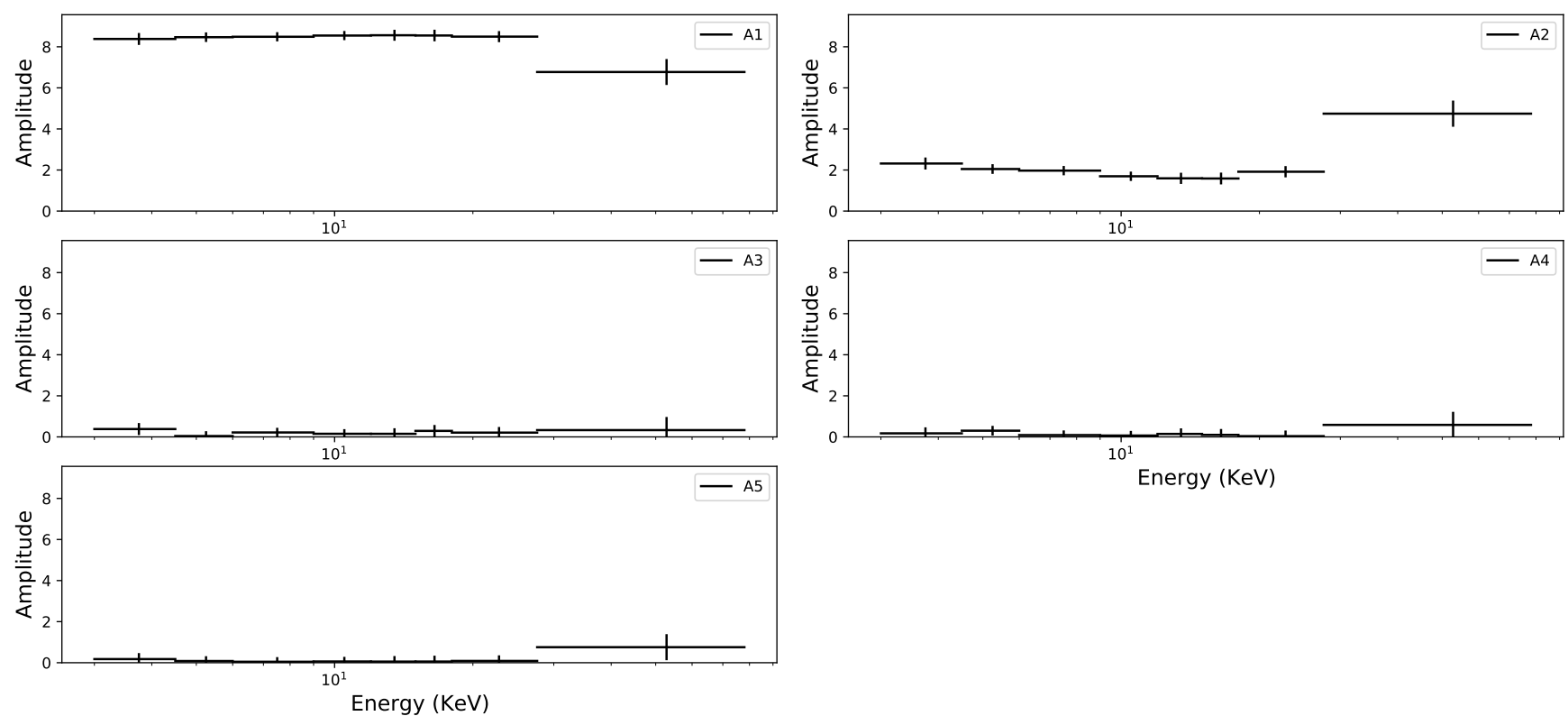

Energy $(\mathrm{KeV})$

Fig. A.7. Variation of the harmonic amplitudes for $4 \mathrm{U} 1901+03$ (observation with OBS ID $=90501324002$ and a luminosity value of $4.84_{-0.02}^{+0.03}$. $\left.10^{37} \mathrm{erg} \cdot \mathrm{s}^{-1}\right)$. The boundaries of the energy ranges are $[3,4.5,6,9,12,15,18,27.6$, and 78] keV. Only the NuSTAR data are represented by black markers.
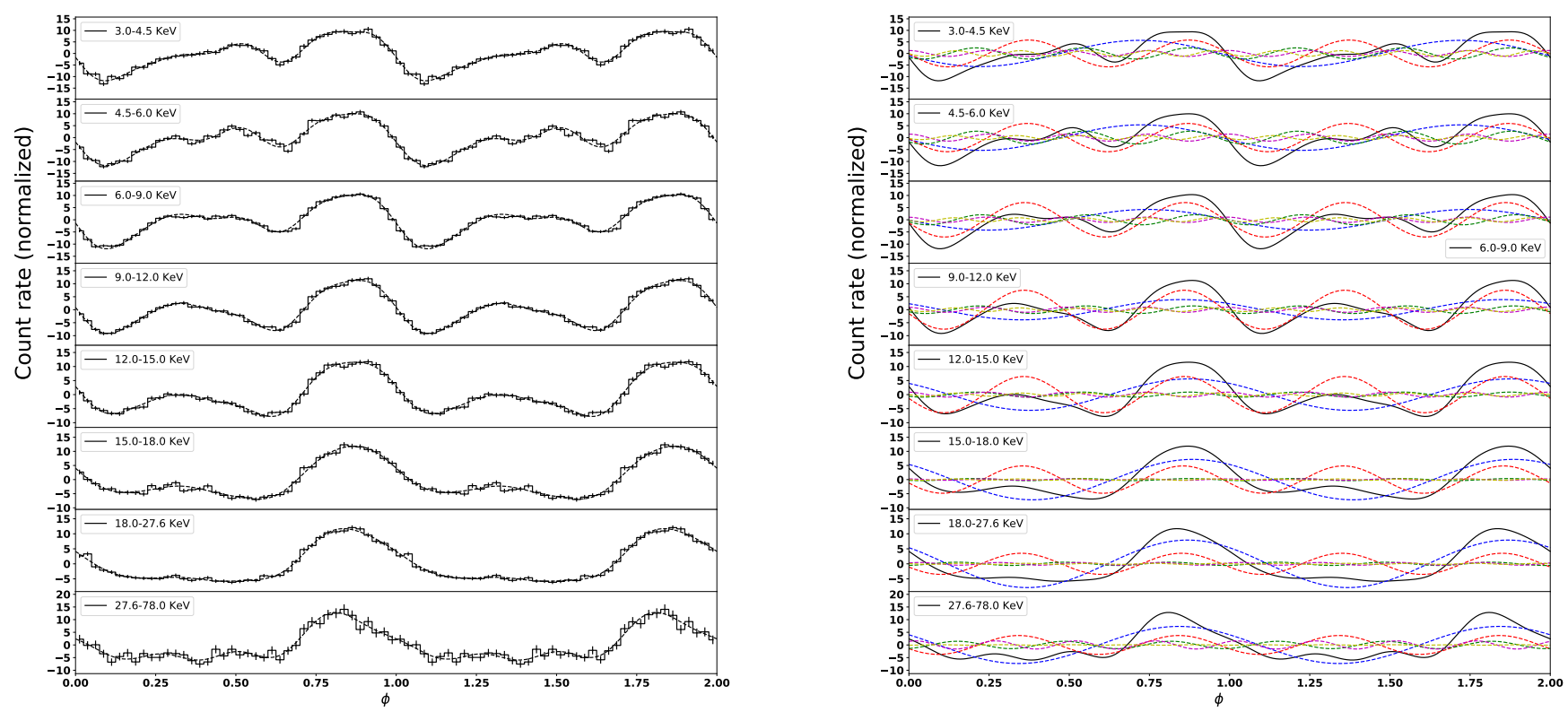

Fig. A.8. Comparison of the observed energy resolved pulse profiles of 4 U 1901+03 (observation with OBS ID=90502307002 and a luminosity value of $1.483_{-0.002}^{+0.002} \cdot 10^{38} \mathrm{erg} \cdot \mathrm{s}^{-1}$ ) and their Fourier fits at different energy bands. Left: Normalized pulse profiles of $4 \mathrm{U} 1901+03$ (observation with OBS ID=90502307002 and a luminosity value of $1.483_{-0.002}^{+0.002} \cdot 10^{38} \mathrm{erg} \cdot \mathrm{s}^{-1}$ ) at different energy ranges based on NuSTAR data. The measured profiles are represented by solid lines, whereas the Fourier fits are represented by dashed lines. The boundaries of the energy ranges are [3, 4.5, 6 , $9,12,15,18,27.6$, and 78$] \mathrm{keV}$. Right: Overall approximate pulse profile (black solid line) and Fourier components 1-5 (coloured dashed lines: blue, red, green, purple, and yellow, respectively). 

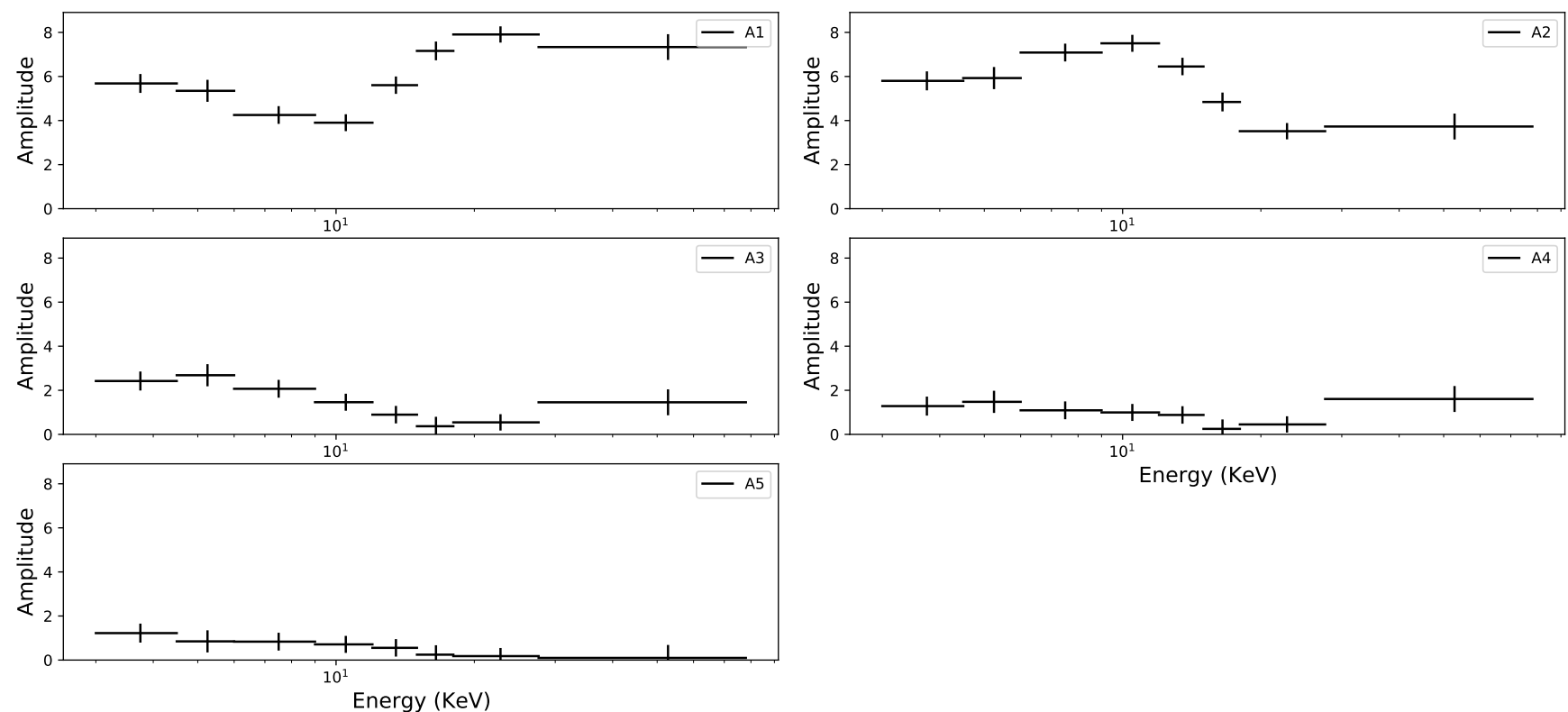

Fig. A.9. Variation of the harmonic amplitudes for $4 \mathrm{U} 1901+03$ (observation with OBS ID $=90502307002$ and a luminosity value of $1.483_{-0.002}^{+0.002}$. $\left.10^{38} \mathrm{erg} \cdot \mathrm{s}^{-1}\right)$. The boundaries of the energy ranges are $[3,4.5,6,9,12,15,18,27.6$, and 78] keV. Only the NuSTAR data are represented by black markers.
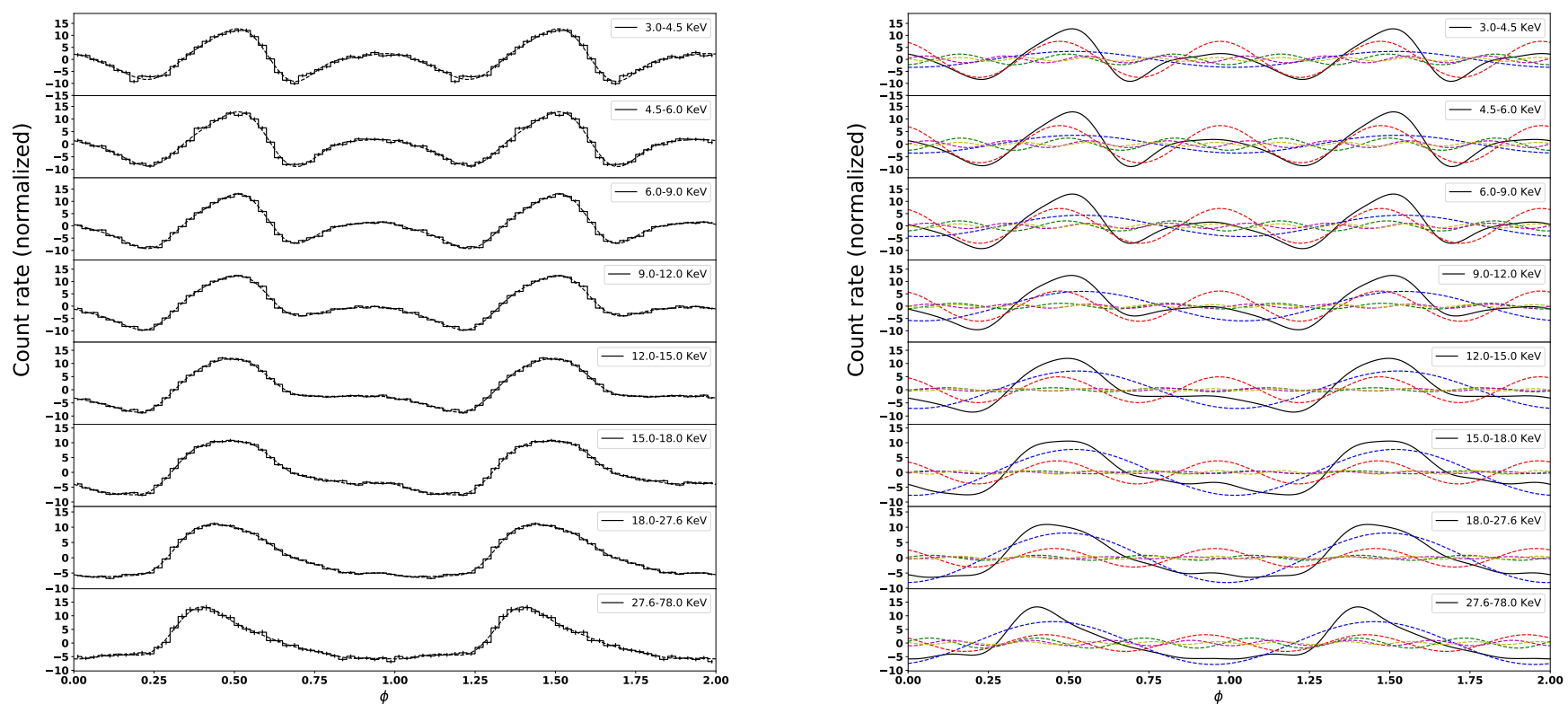

Fig. A.10. Comparison of the observed energy resolved pulse profiles of 4U 1901+03 (observation with OBS ID=90501305001 and a luminosity value of $1.242_{-0.005}^{+0.010} \cdot 10^{38} \mathrm{erg} \cdot \mathrm{s}^{-1}$ ) and their Fourier fits at different energy bands. Left: Normalized pulse profiles of $4 \mathrm{U} 1901+03$ (observation with OBS ID=90501305001 and a luminosity value of $1.242_{-0.005}^{+0.010} \cdot 10^{38} \mathrm{erg} \cdot \mathrm{s}^{-1}$ ) at different energy ranges based on NuSTAR data. The measured profiles are represented by solid lines, whereas the Fourier fits are represented by dashed lines. The boundaries of the energy ranges are [3, 4.5, 6 , $9,12,15,18,27.6$, and 78] keV. Right: Overall approximate pulse profile (black solid line) and Fourier components 1-5 (coloured dashed lines: blue, red, green, purple, and yellow, respectively). 
J. Alonso-Hernández et al.: Common patterns in pulse profiles of HMXBs
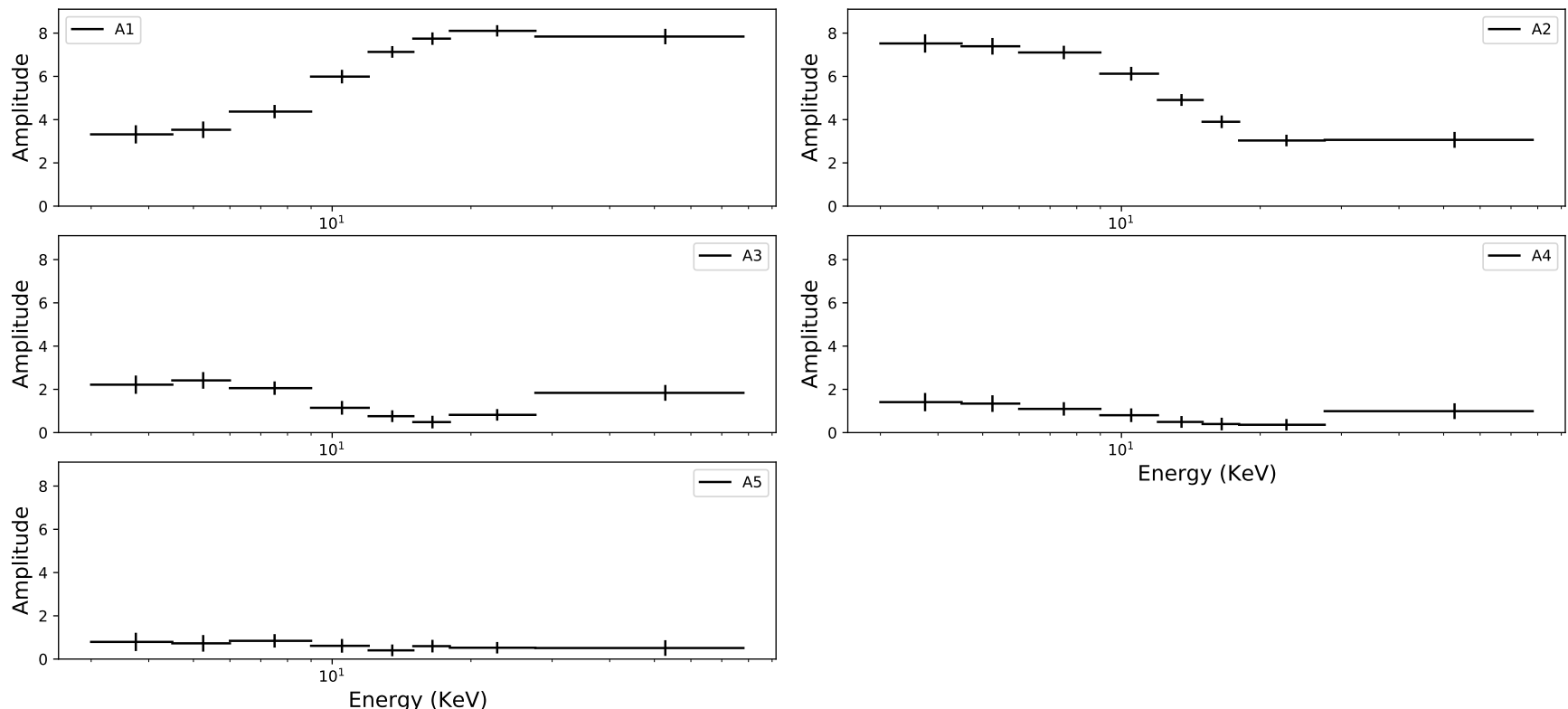

Energy (KeV)

Fig. A.11. Variation of the harmonic amplitudes for $4 \mathrm{U} 1901+03$ (observation with OBS ID=90501305001 and a luminosity value of $1.242_{-0.005}^{+0.010}$. $\left.10^{38} \mathrm{erg} \cdot \mathrm{s}^{-1}\right)$. The boundaries of the energy ranges are $[3,4.5,6,9,12,15,18,27.6$, and 78] keV. Only the NuSTAR data are represented by black markers.
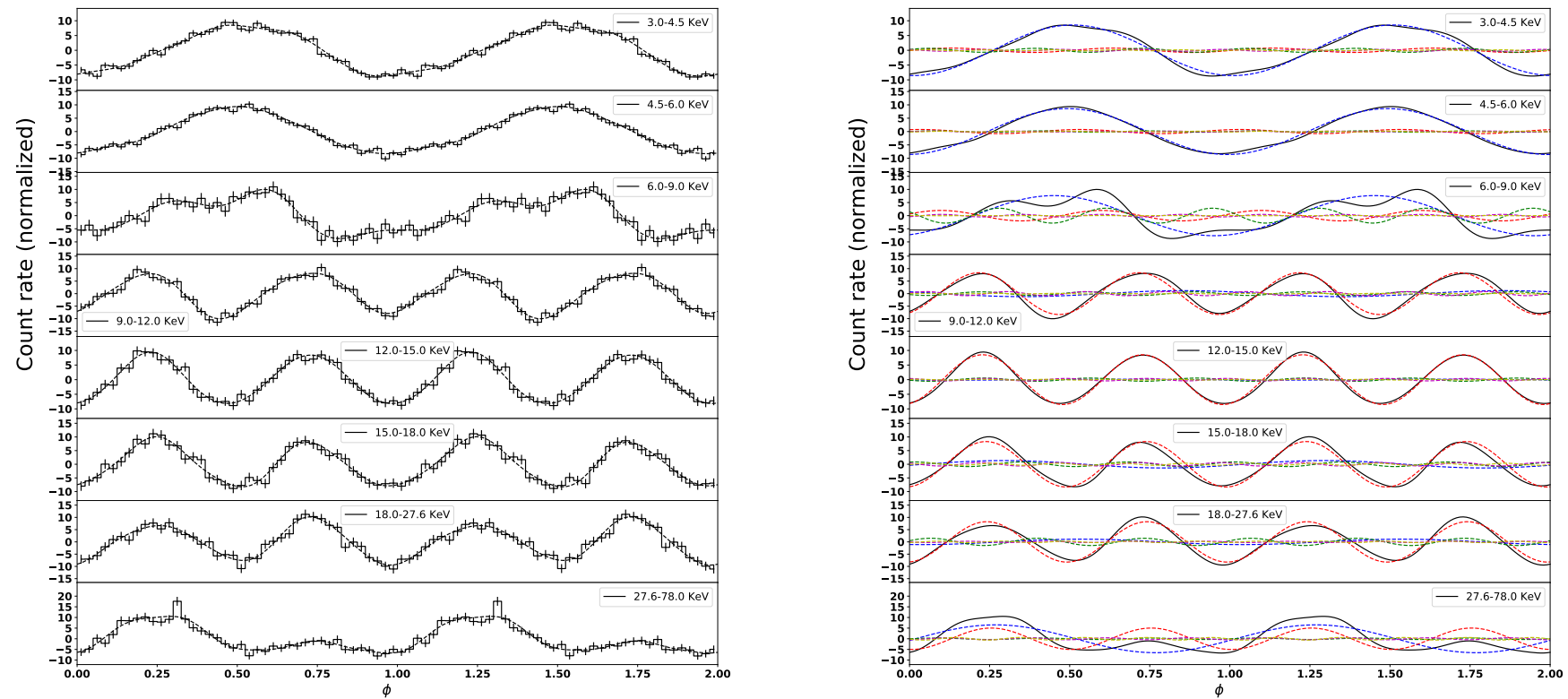

Fig. A.12. Comparison of the observed energy resolved pulse profiles of V 0332+53 (observation with OBS ID=80102002002 and a luminosity value of $\left.(1.24-1.74) \cdot 10^{38} \mathrm{erg} \cdot \mathrm{s}^{-1}\right)$ and their Fourier fits at different energy bands. Left: Normalized pulse profiles of V $0332+53$ (observation with OBS ID=80102002002 and a luminosity value of $\left.(1.24-1.74) \cdot 10^{38} \mathrm{erg} \cdot \mathrm{s}^{-1}\right)$ at different energy ranges based on NuSTAR data. The measured profiles are represented by solid lines, whereas the Fourier fits are represented by dashed lines. The boundaries of the energy ranges are [3, 4.5, 6 , $9,12,15,18,27.6$, and 78$] \mathrm{keV}$. Right: Overall approximate pulse profile (black solid line) and Fourier components 1-5 (coloured dashed lines: blue, red, green, purple, and yellow, respectively). 

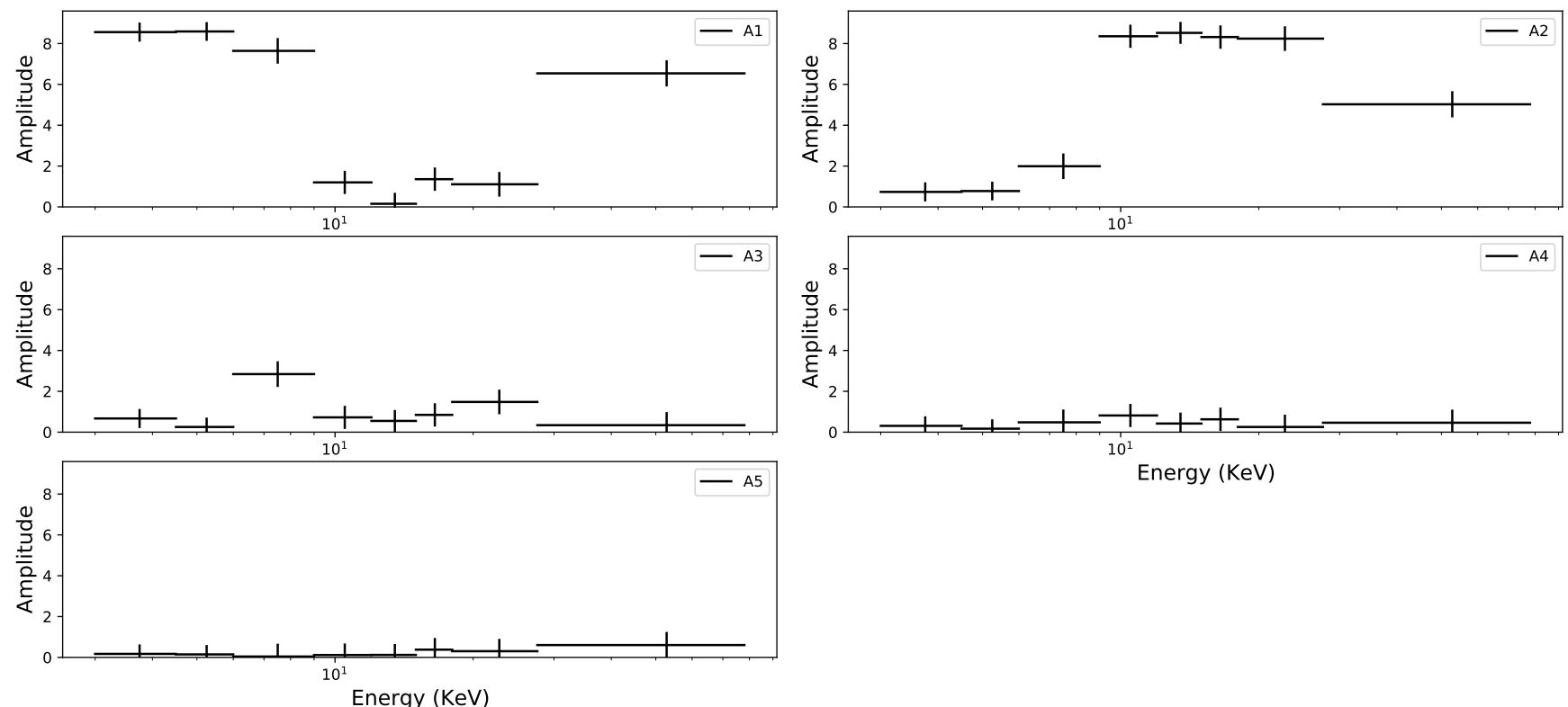

Energy $(\mathrm{KeV})$

Fig. A.13. Variation of the harmonic amplitudes for V 0332+53 (observation with OBS ID=80102002002 and a luminosity value of (1.24 - 1.74) . $\left.10^{38} \mathrm{erg} \cdot \mathrm{s}^{-1}\right)$. The boundaries of the energy ranges are $[3,4.5,6,9,12,15,18,27.6$, and 78] keV. Only the NuSTAR data are represented by black markers.
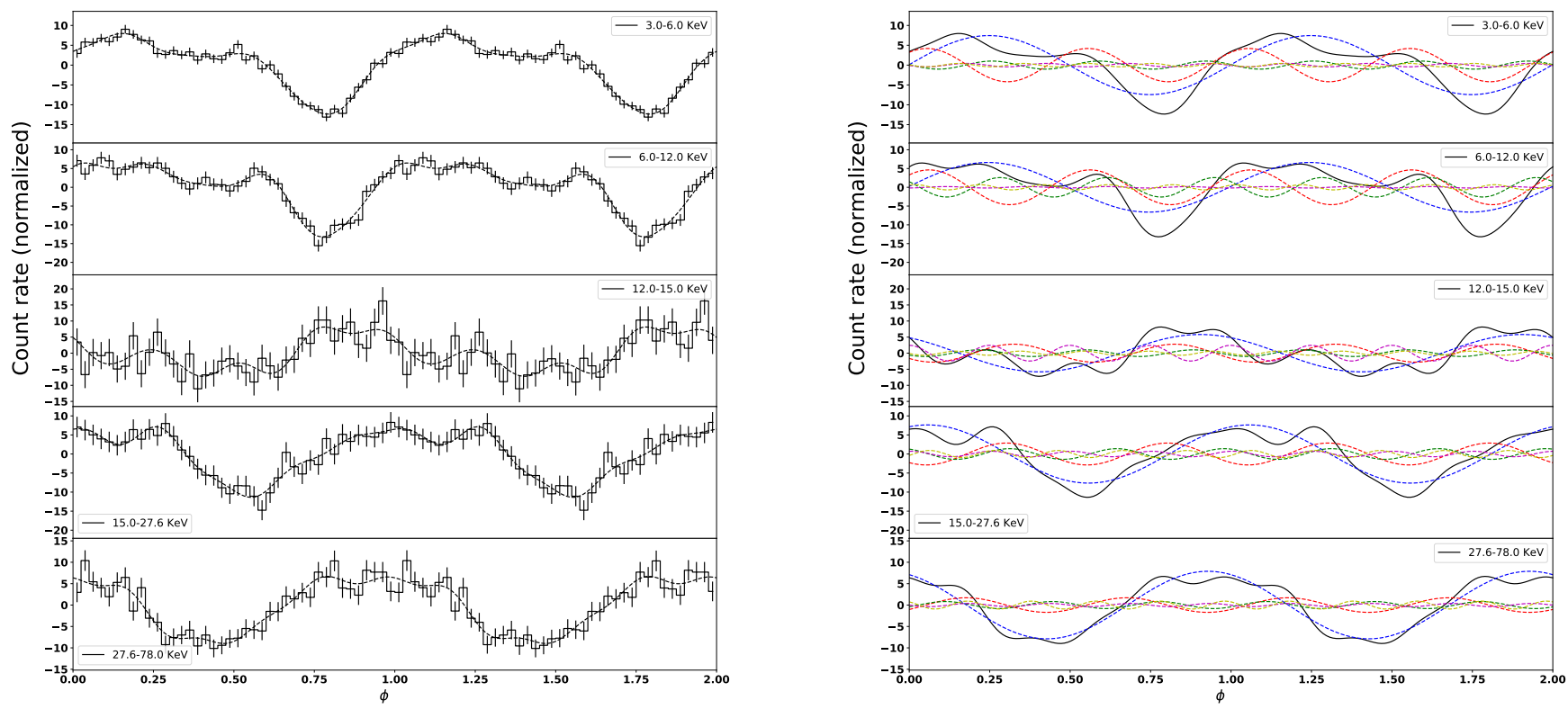

Fig. A.14. Comparison of the observed energy resolved pulse profiles of V 0332+53 (observation with OBS ID=80102002004 and a luminosity value of $\left.(5.7-9.5) \cdot 10^{37} \mathrm{erg} \cdot \mathrm{s}^{-1}\right)$ and their Fourier fits at different energy bands. Left: Normalized pulse profiles of V $0332+53$ (observation with OBS ID=80102002004 and a luminosity value of $\left.(5.7-9.5) \cdot 10^{37} \mathrm{erg} \cdot \mathrm{s}^{-1}\right)$ at different energy ranges based on NuSTAR data. The measured profiles are represented by solid lines, whereas the Fourier fits are represented by dashed lines. The boundaries of the energy ranges are [3, 6, 12, 15, 27.6, and 78] keV. Right: Overall approximate pulse profile (black solid line) and Fourier components 1-5 (coloured dashed lines: blue, red, green, purple, and yellow, respectively). 
J. Alonso-Hernández et al.: Common patterns in pulse profiles of HMXBs
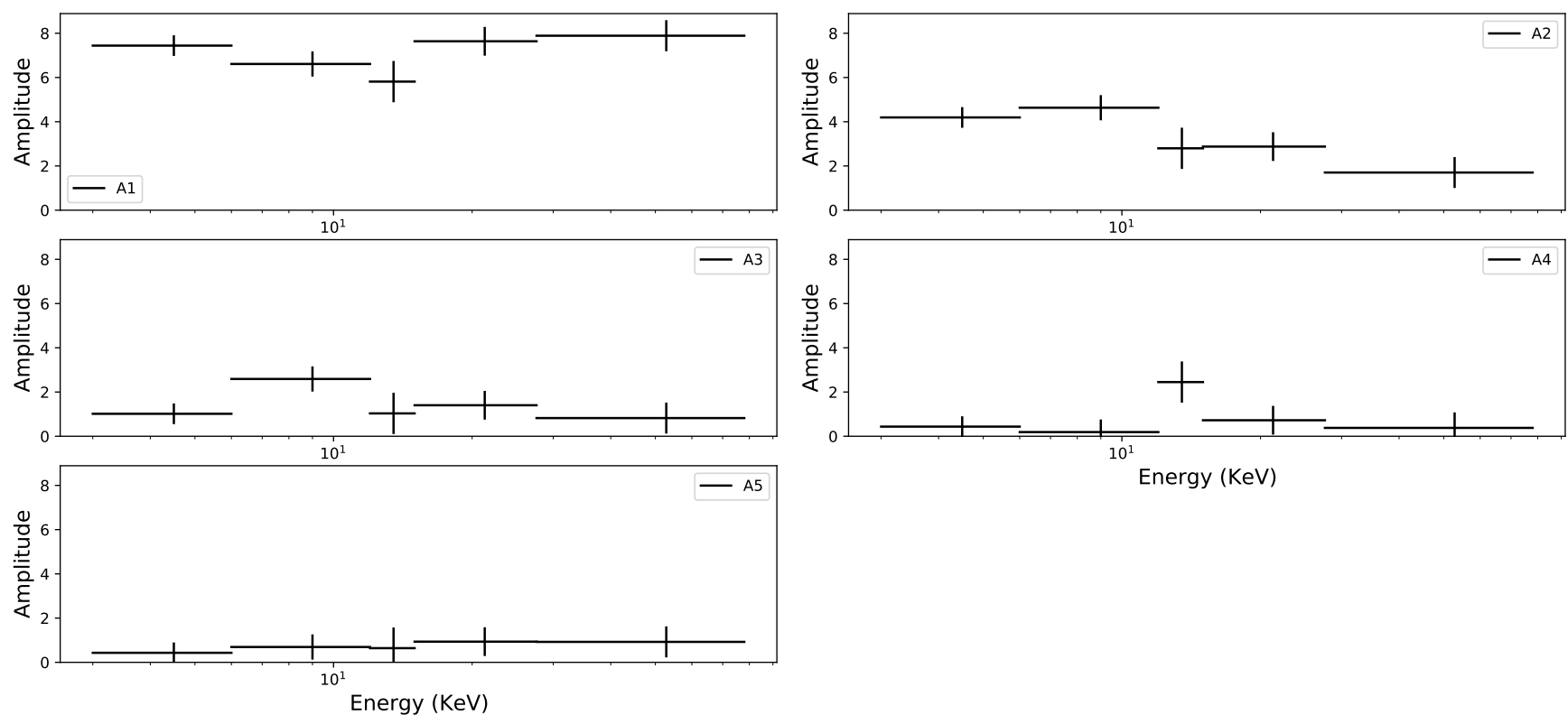

Energy $(\mathrm{KeV})$

Fig. A.15. Variation of the harmonic amplitudes for V $0332+53$ (observation with OBS ID=80102002004 and a luminosity value of (5.7 - 9.5) . $\left.10^{37} \mathrm{erg} \cdot \mathrm{s}^{-1}\right)$. The boundaries of the energy ranges are $[3,6,12,15,27.6$, and 78] keV. Only the NuSTAR data are represented by black markers.
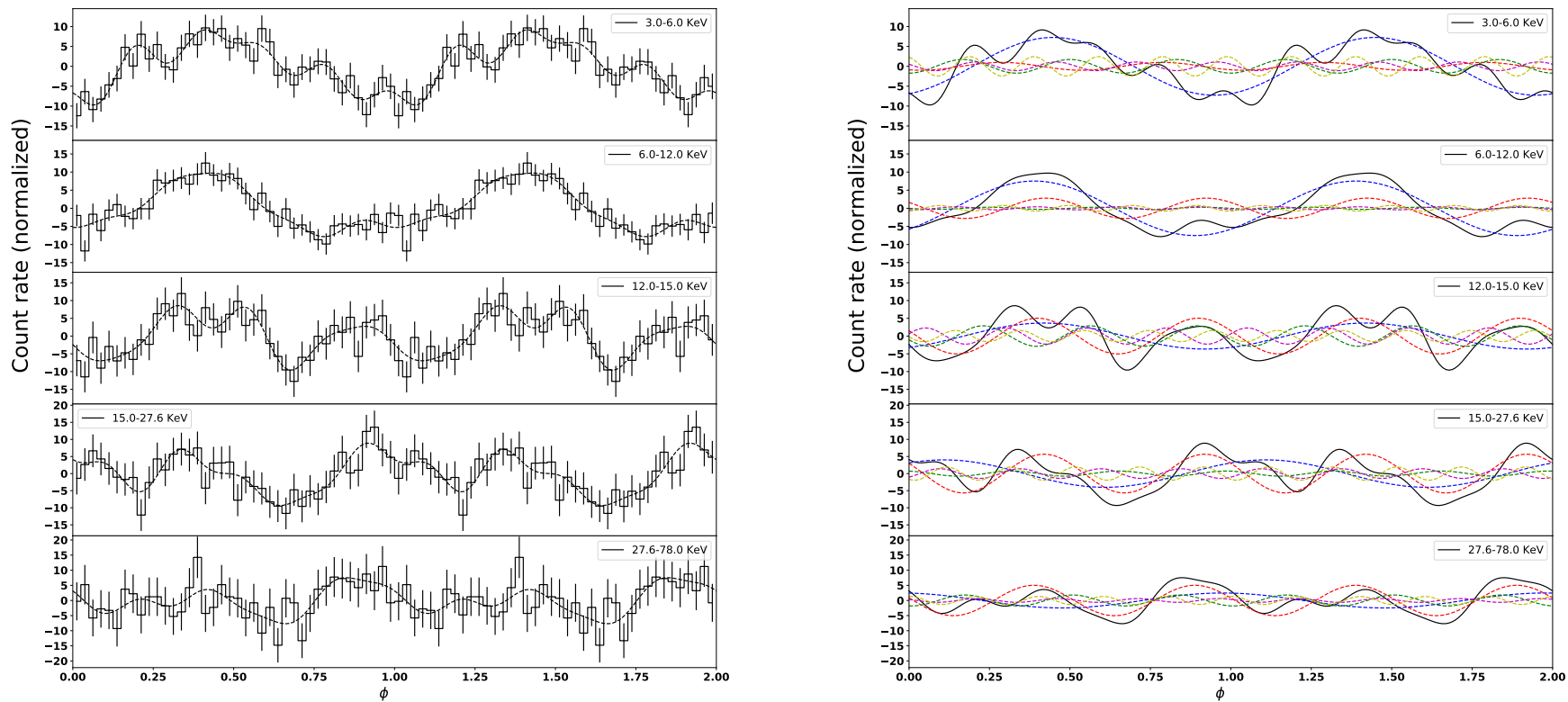

Fig. A.16. Comparison of the observed energy resolved pulse profiles of V $0332+53$ (observation with OBS ID=80102002010 and a luminosity value of $\left.(5.3-14) \cdot 10^{36} \mathrm{erg} \cdot \mathrm{s}^{-1}\right)$ and their Fourier fits at different energy bands. Left: Normalized pulse profiles of V $0332+53$ (observation with OBS ID=80102002010 and a luminosity value of $\left.(5.3-14) \cdot 10^{36} \mathrm{erg} \cdot \mathrm{s}^{-1}\right)$ at different energy ranges based on $N u S T A R$ data. The measured profiles are represented by solid lines, whereas the Fourier fits are represented by dashed lines. The boundaries of the energy ranges are [3, 6, 12, 15, 27.6, and 78] keV. Right: Overall approximate pulse profile (black solid line) and Fourier components 1-5 (coloured dashed lines: blue, red, green, purple, and yellow, respectively). 

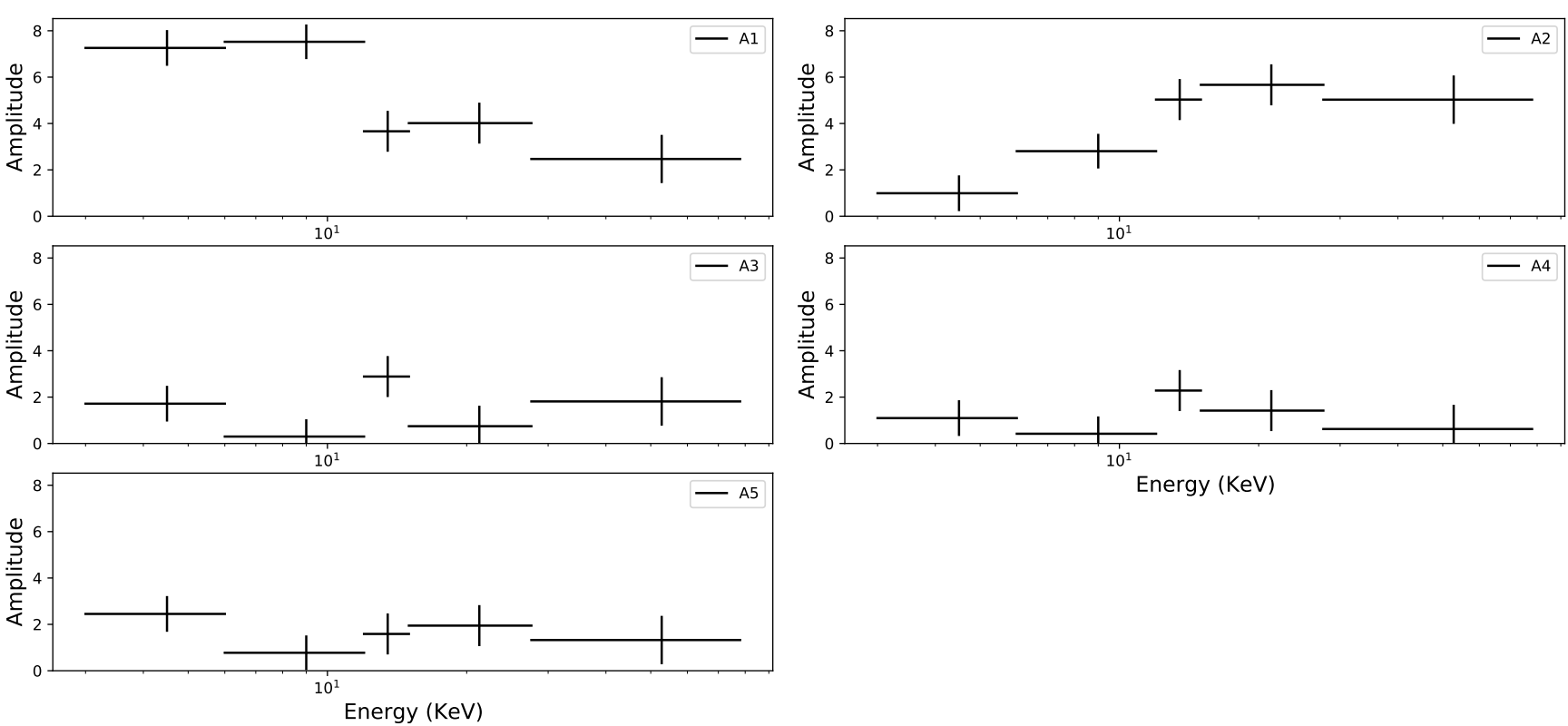

Energy (KeV)

Fig. A.17. Variation of the harmonic amplitudes for V 0332+53 (observation with OBS ID=80102002010 and a luminosity value of (5.3 - 14) . $\left.10^{36} \mathrm{erg} \cdot \mathrm{s}^{-1}\right)$. The boundaries of the energy ranges are $[3,6,12,15,27.6$, and 78$] \mathrm{keV}$. Only the NuSTAR data are represented by black markers.
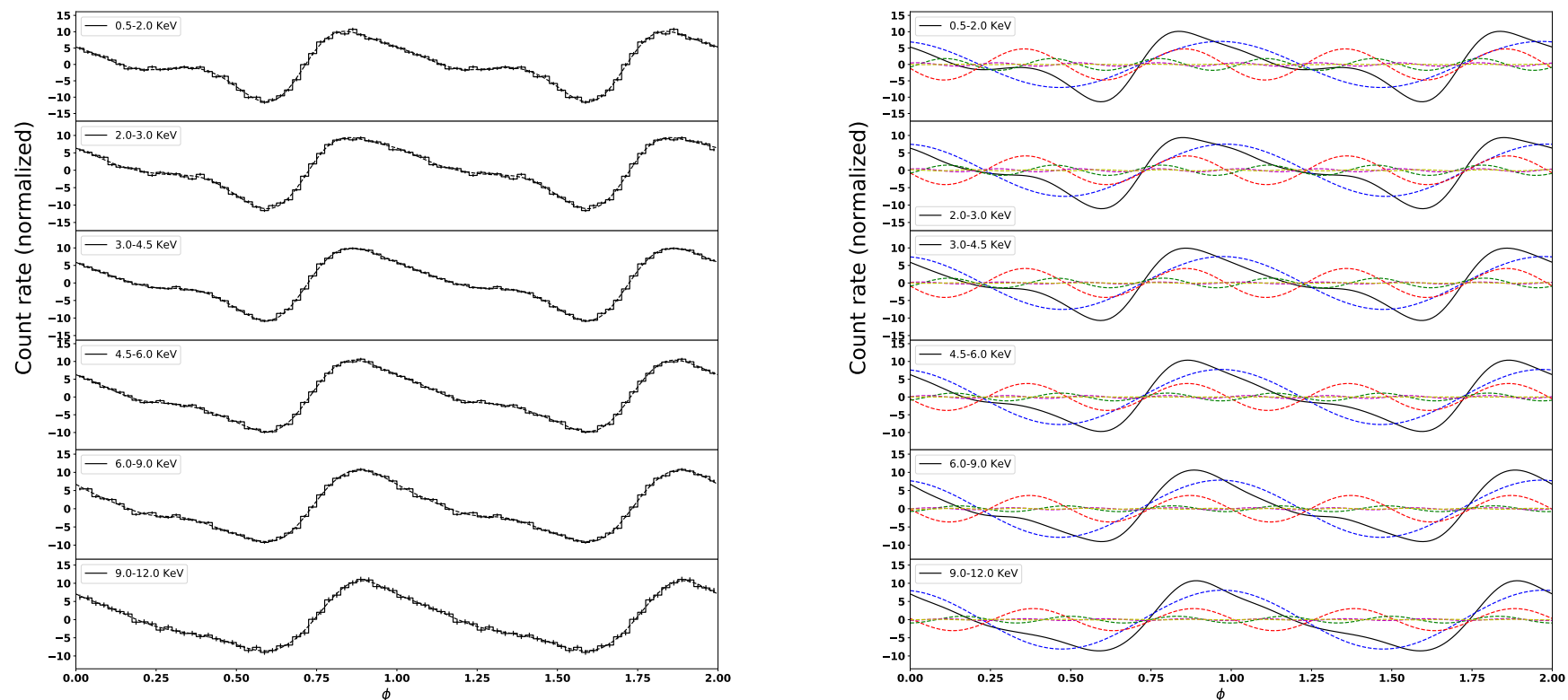

Fig. A.18. Comparison of the observed energy resolved pulse profiles of Cen X-3 and their Fourier fits at different energy bands. Left: Normalized pulse profiles of Cen X-3 at different energy ranges based on XMM-Newton data. The measured profiles are represented by solid lines, whereas the Fourier fits are represented by dashed lines. The boundaries of the energy ranges are $[0.5,2,3,4.5,6,9,12] \mathrm{keV}$. Right: Overall approximate pulse profile (black solid line) and Fourier components 1-5 (coloured dashed lines: blue, red, green, purple, and yellow, respectively). 
J. Alonso-Hernández et al.: Common patterns in pulse profiles of HMXBs
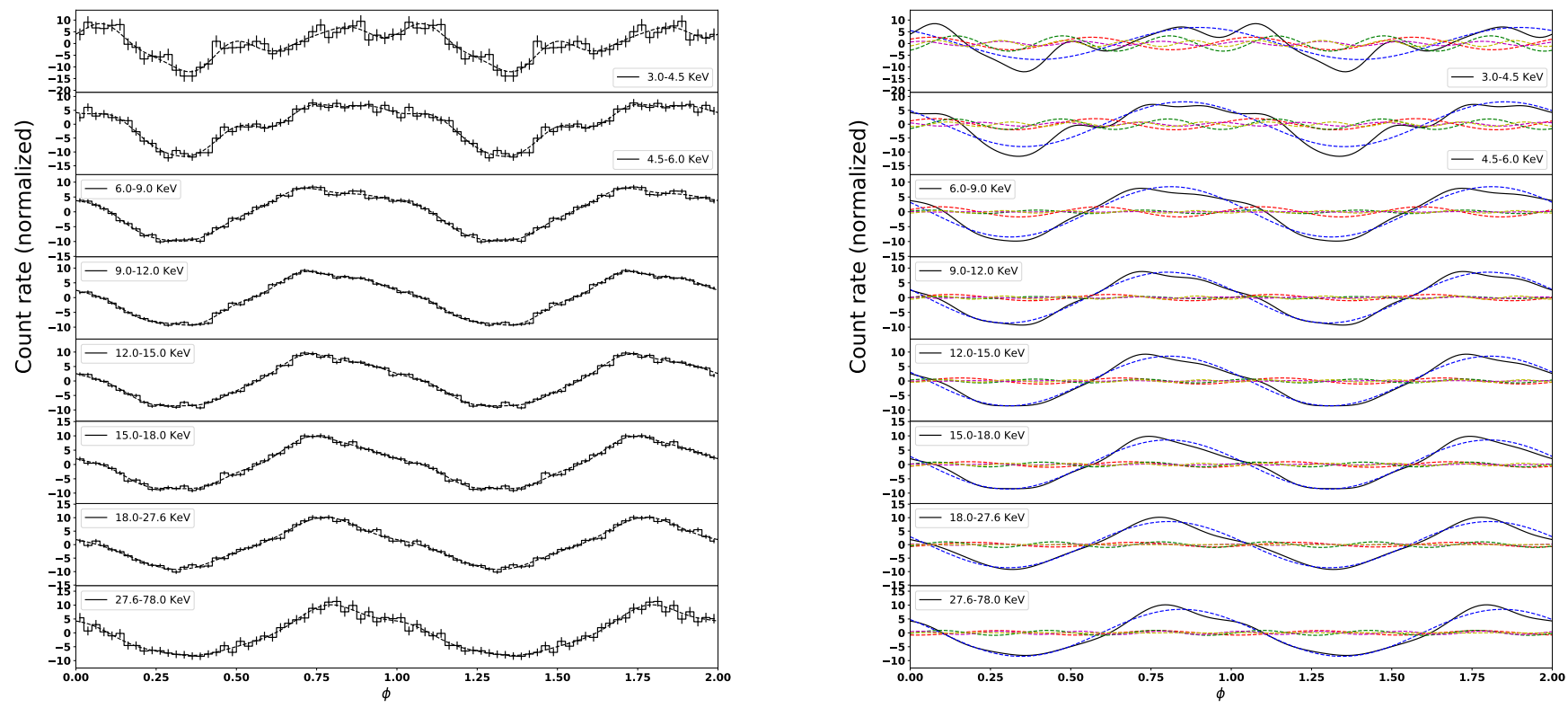

Fig. A.19. Comparison of the observed energy resolved pulse profiles of Cen X-3 and their Fourier fits at different energy bands. Left: Normalized pulse profiles of Cen X-3 at different energy ranges based on NuSTAR data. The measured profiles are represented by solid lines, whereas the Fourier fits are represented by dashed lines. The boundaries of the energy ranges are $[3,4.5,6,9,12,15,18,27.6$, and 78] keV. Right: Overall approximate pulse profile (black solid line) and Fourier components 1-5 (coloured dashed lines: blue, red, green, purple, and yellow, respectively).
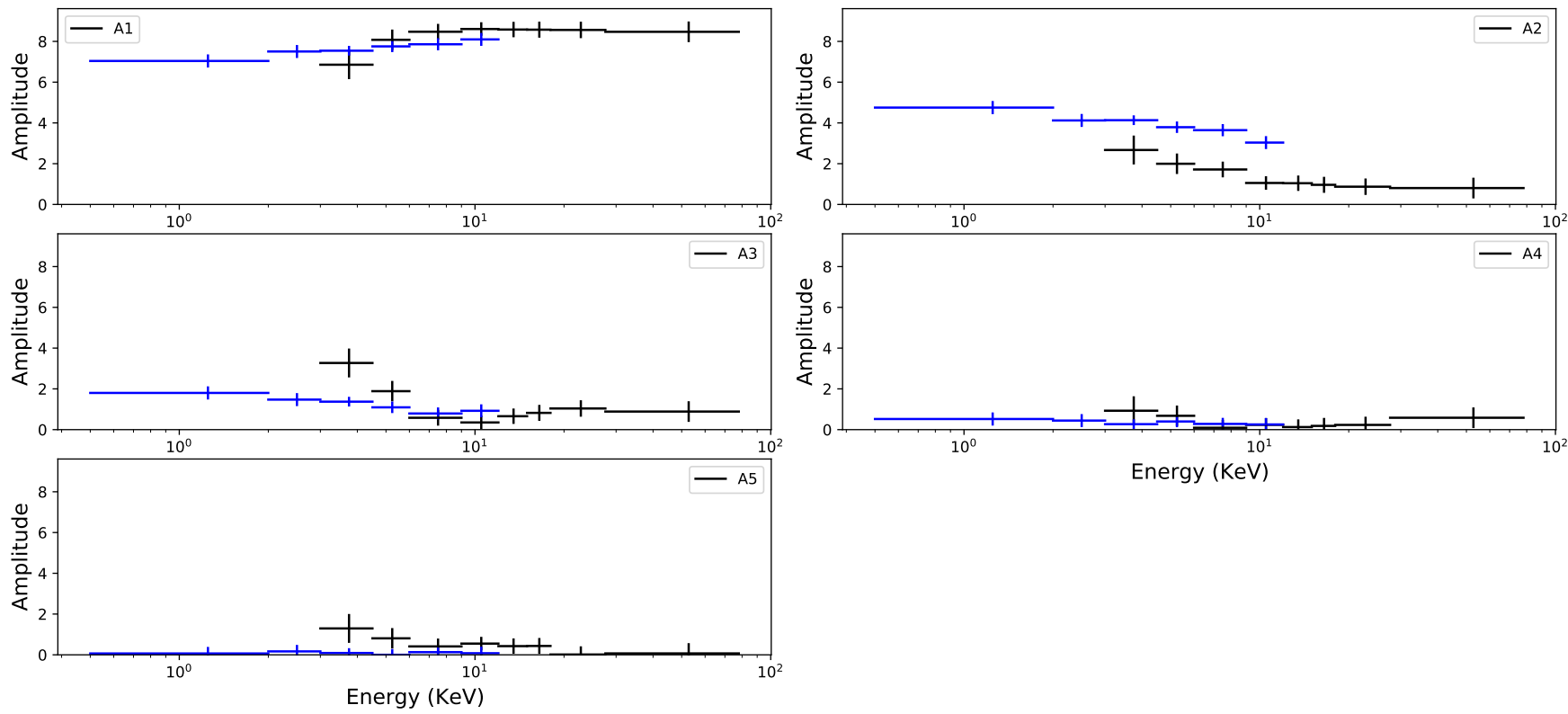

Fig. A.20. Variation of the harmonic amplitudes for Cen X-3. The boundaries of the energy ranges are $[0.5,2,3,4.5,6,9,12,15,18,27.6$, and 78] keV. The XMM-Newton data are represented by blue markers and NUSTAR data are represented by black markers. 

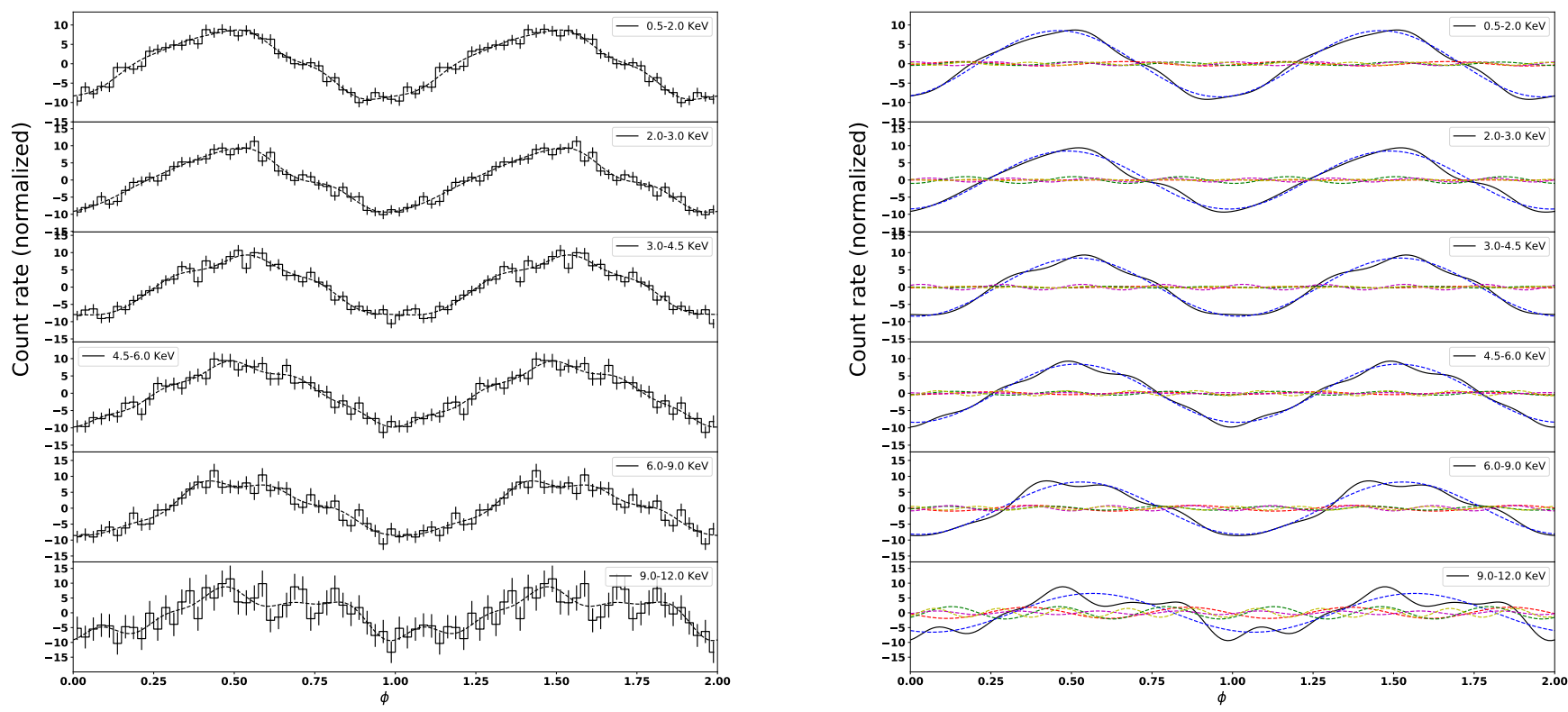

Fig. A.21. Comparison of the observed energy resolved pulse profiles of LMC X-4 and their Fourier fits at different energy bands. Left: Normalized pulse profiles of LMC X-4 at different energy ranges based on XMM-Newton data. The measured profiles are represented by solid lines, whereas the Fourier fits are represented by dashed lines. The boundaries of the energy ranges are $[0.5,2,3,4.5,6,9,12]$ keV. Right: Overall approximate pulse profile (black solid line) and Fourier components 1-5 (coloured dashed lines: blue, red, green, purple, and yellow, respectively).
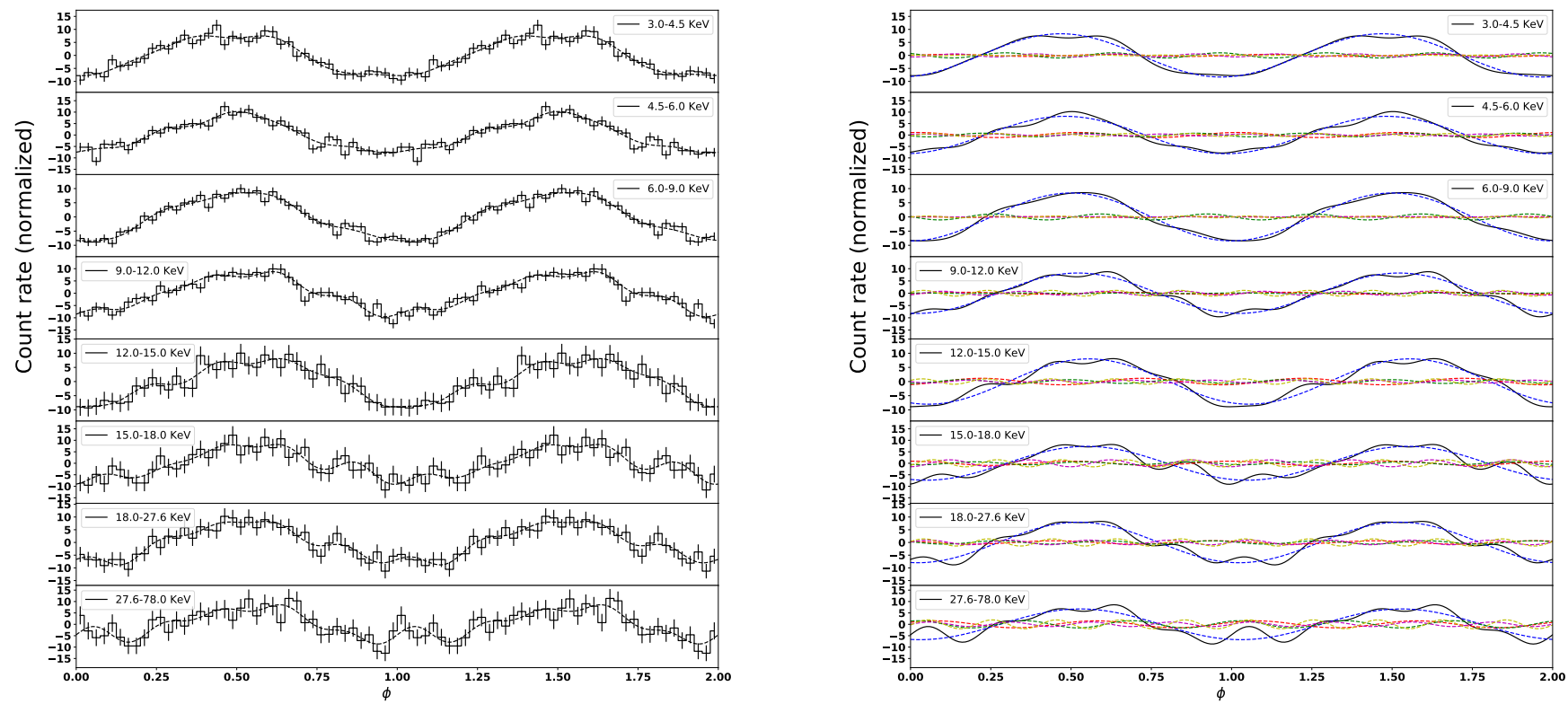

Fig. A.22. Comparison of the observed energy resolved pulse profiles of LMC X-4 and their Fourier fits at different energy bands. Left: Normalized pulse profiles of LMC X-4 at different energy ranges based on NuSTAR data. The measured profiles are represented by solid lines, whereas the Fourier fits are represented by dashed lines. The boundaries of the energy ranges are $[3,4.5,6,9,12,15,18,27.6$, and 78] keV. Right: Overall approximate pulse profile (black solid line) and Fourier components 1-5 (coloured dashed lines: blue, red, green, purple, and yellow, respectively). 

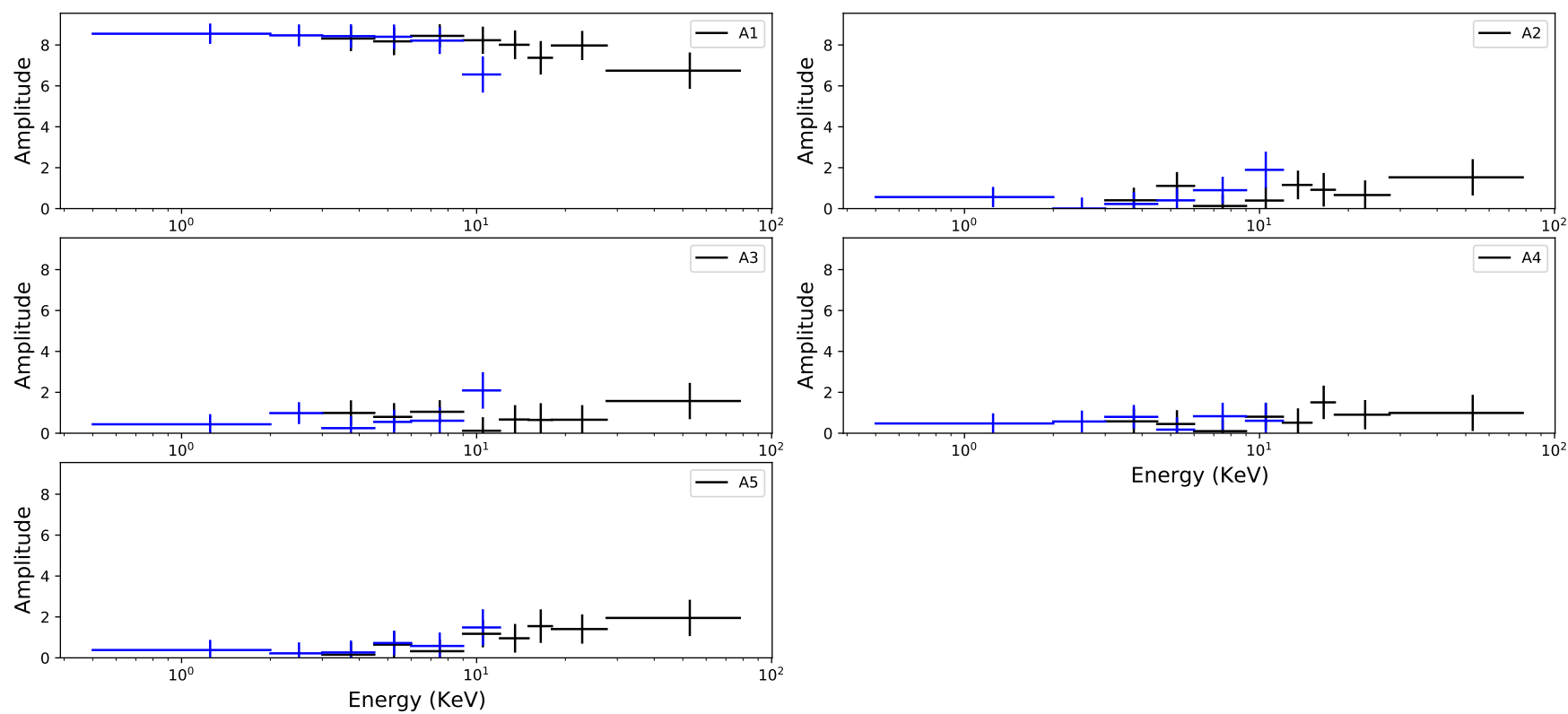

Energy $(\mathrm{KeV})$

Fig. A.23. Variation of the harmonic amplitudes for LMC X-4. The boundaries of the energy ranges are $[0.5,2,3,4.5,6,9,12,15,18,27.6$, and $78] \mathrm{keV}$. The XMM-Newton data are represented by blue markers and NuSTAR data are represented by black markers.
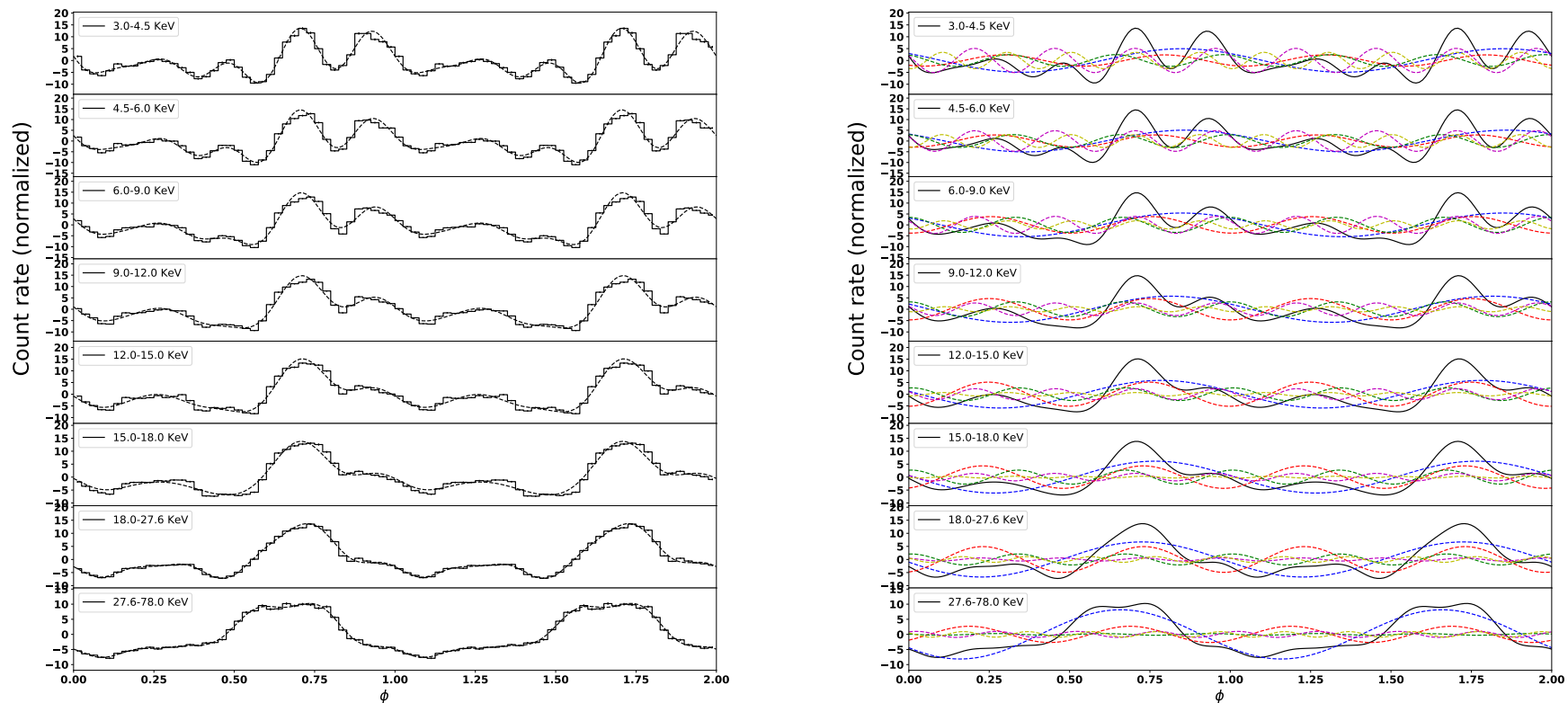

Fig. A.24. Comparison of the observed energy resolved pulse profiles of GRO J2058+42 and their Fourier fits at different energy bands. Left: Normalized pulse profiles of GRO J2058+42 at different energy ranges based on NuSTAR data. The measured profiles are represented by solid lines, whereas the Fourier fits are represented by dashed lines. The boundaries of the energy ranges are $[3,4.5,6,9,12,15,18,27.6$, and 78] keV. Right: Overall approximate pulse profile (black solid line) and Fourier components 1-5 (coloured dashed lines: blue, red, green, purple, and yellow, respectively). 

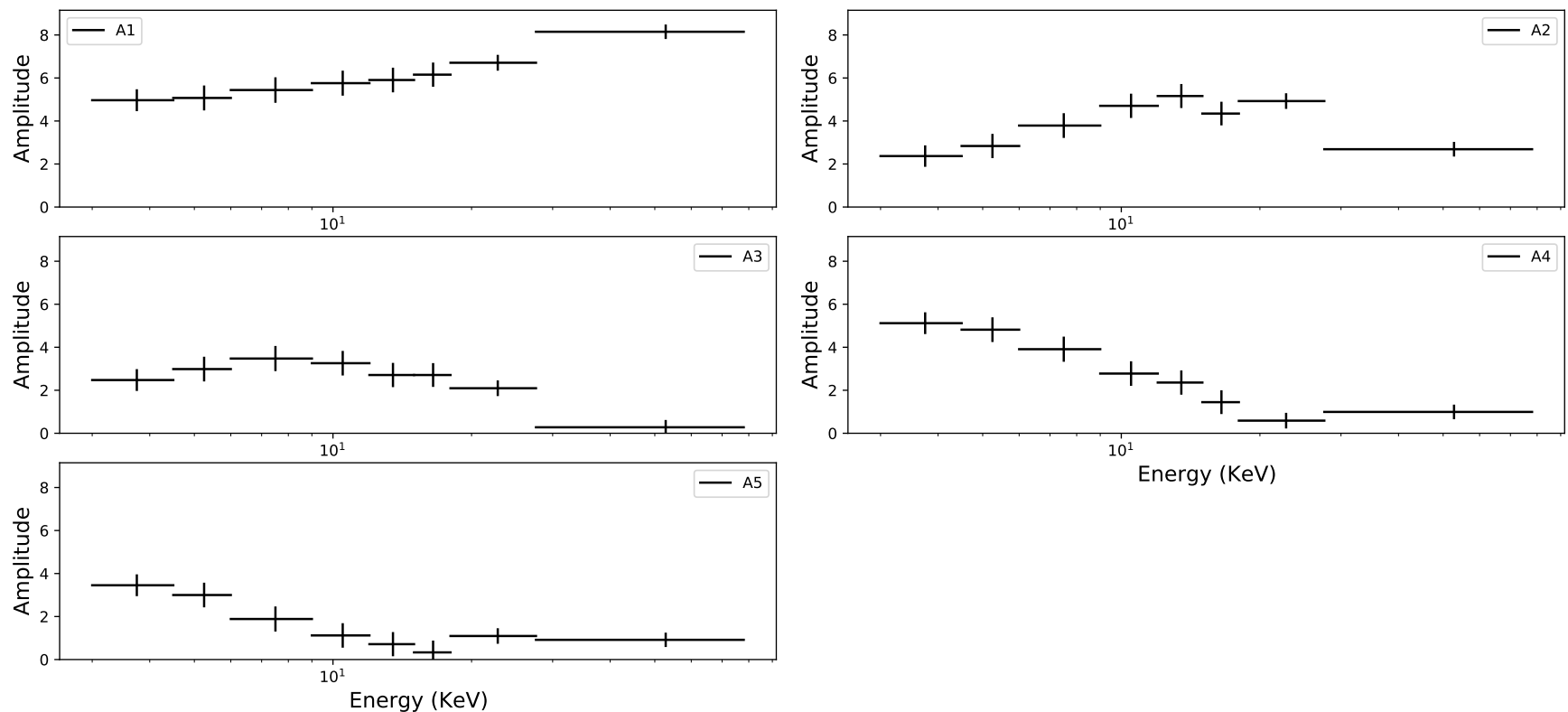

Energy (KeV)

Fig. A.25. Variation of the harmonic amplitudes for GRO J2058+42. The boundaries of the energy ranges are [3, 4.5, 6, 9, 12, 15, 18, 27.6, and 78 ] keV. Only the NuSTAR data are represented by black markers.
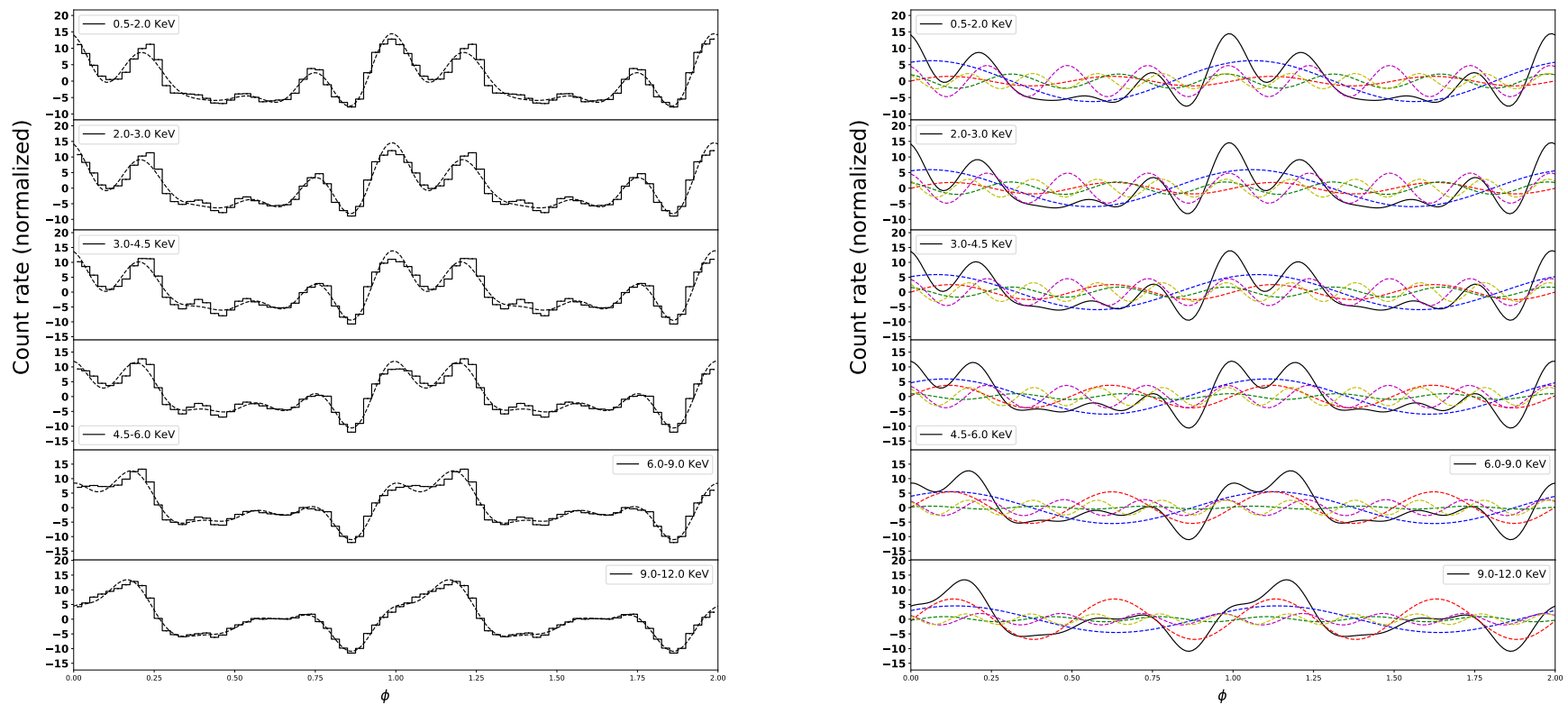

Fig. A.26. Comparison of the observed energy resolved pulse profiles of Vela X-1 and their Fourier fits at different energy bands. Left: Normalized pulse profiles of Vela X-1 at different energy ranges based on XMM-Newton data. The measured profiles are represented by solid lines, whereas the Fourier fits are represented by dashed lines. The boundaries of the energy ranges are $[0.5,2,3,4.5,6,9,12] \mathrm{keV}$. Right: Overall approximate pulse profile (black solid line) and Fourier components 1-5 (coloured dashed lines: blue, red, green, purple, and yellow, respectively). 

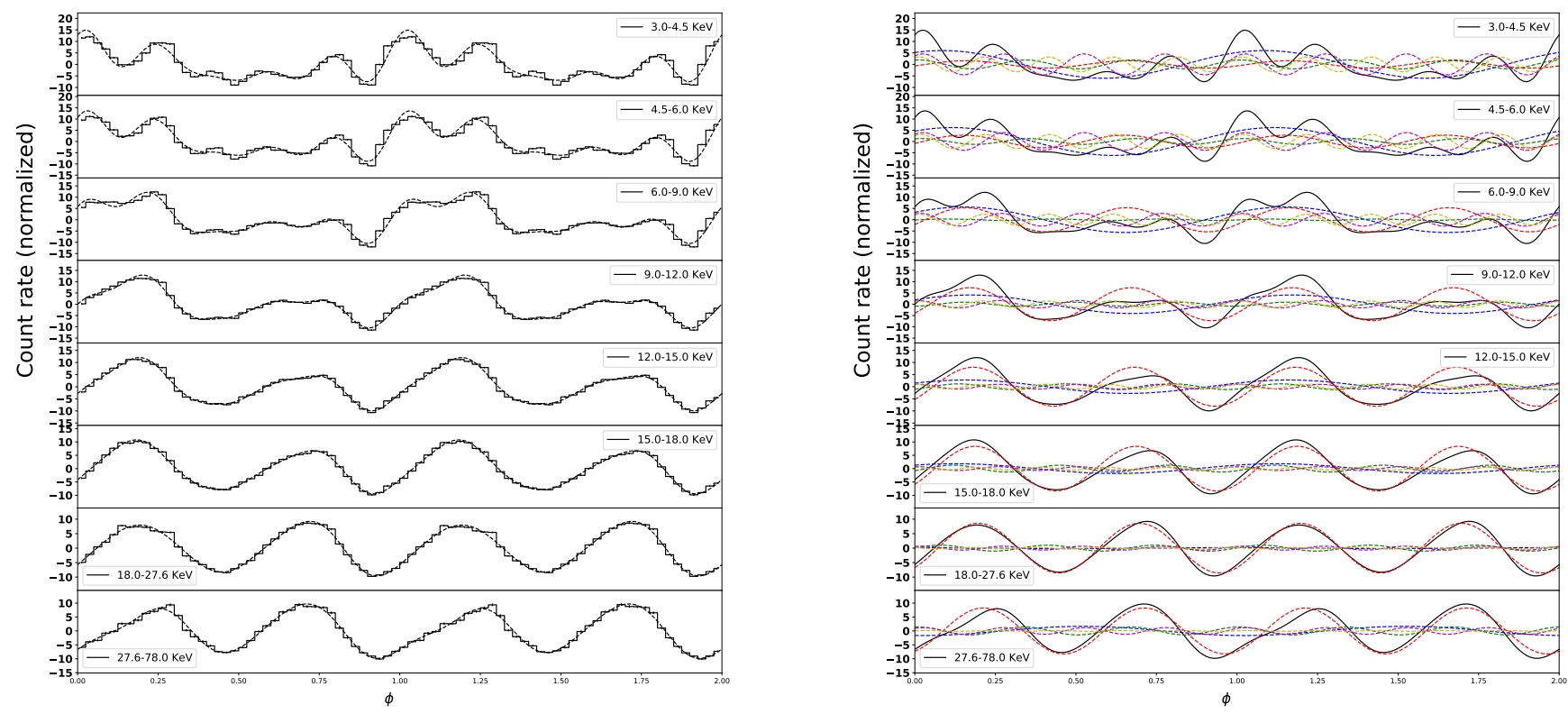

Fig. A.27. Comparison of the observed energy resolved pulse profiles of Vela X-1 and their Fourier fits at different energy bands. Left: Normalized pulse profiles of Vela X-1 at different energy ranges based on NuSTAR data. The measured profiles are represented by solid lines, whereas the Fourier fits are represented by dashed lines. The boundaries of the energy ranges are $[3,4.5,6,9,12,15,18,27.6$, and 78] keV. Right: Overall approximate pulse profile (black solid line) and Fourier components 1-5 (coloured dashed lines: blue, red, green, purple, and yellow, respectively).
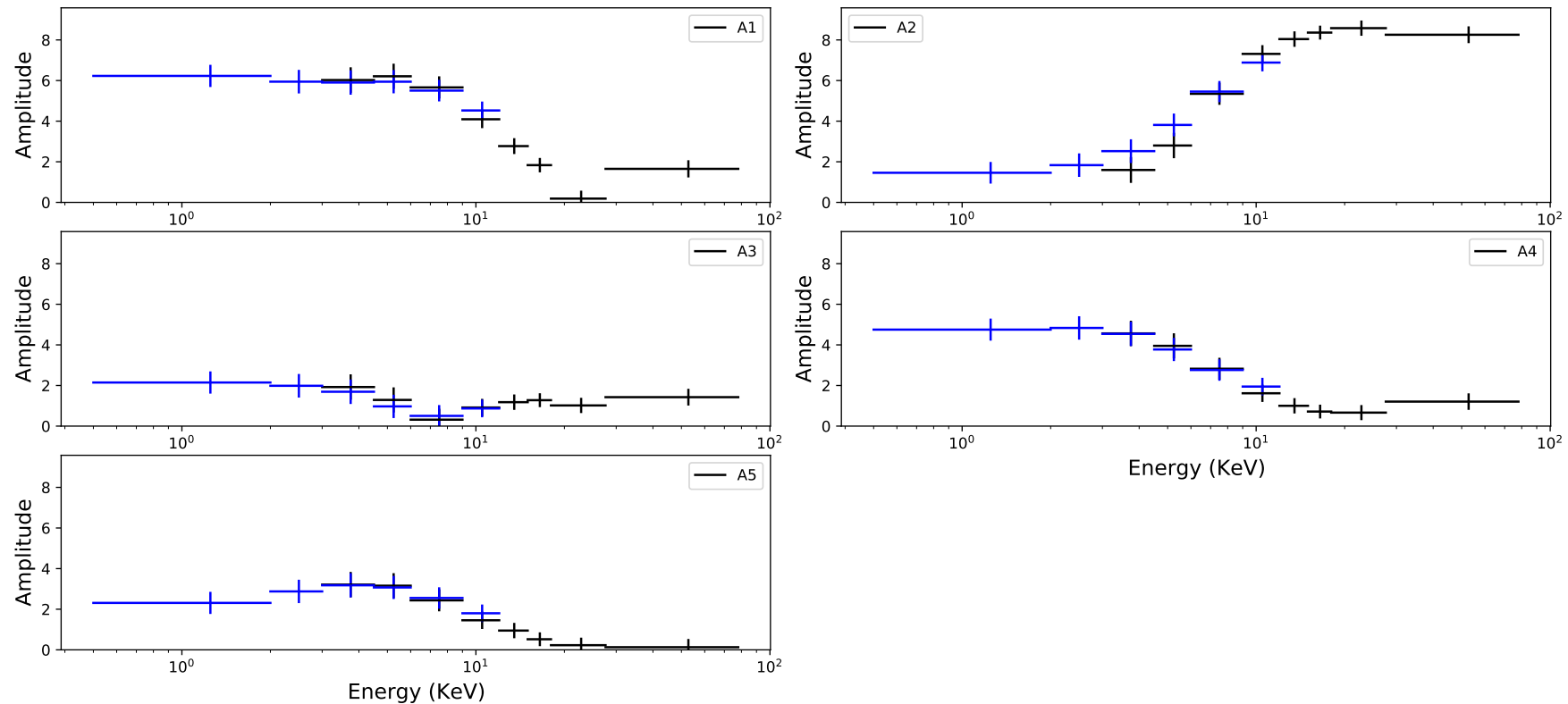

Energy $(\mathrm{KeV})$

Fig. A.28. Variation of the harmonic amplitudes for Vela X-1. The boundaries of the energy ranges are $[0.5,2,3,4.5,6,9,12,15,18,27.6$, and 78$] \mathrm{keV}$. The XMM-Newton data are represented by blue markers and NuSTAR data are represented by black markers. 

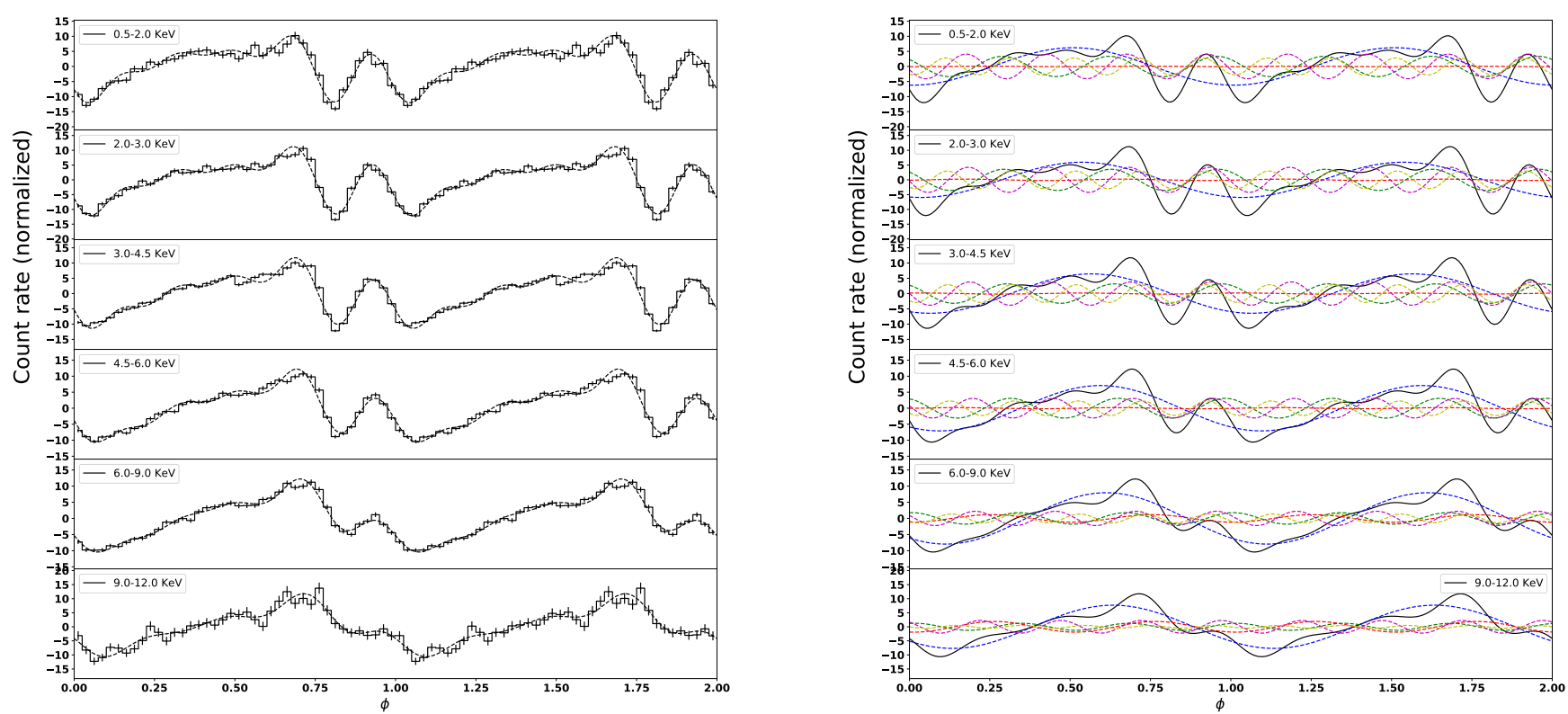

Fig. A.29. Comparison of the observed energy resolved pulse profiles of 1E1145.1-6141 and their Fourier fits at different energy bands. Left: Normalized pulse profiles of 1E1145.1-6141 at different energy ranges based on XMM-Newton data. The measured profiles are represented by solid lines, whereas the Fourier fits are represented by dashed lines. The boundaries of the energy ranges are [0.5, 2, 3, 4.5, 6, 9, 12] keV. Right: Overall approximate pulse profile (black solid line) and Fourier components 1-5 (coloured dashed lines: blue, red, green, purple, and yellow, respectively).
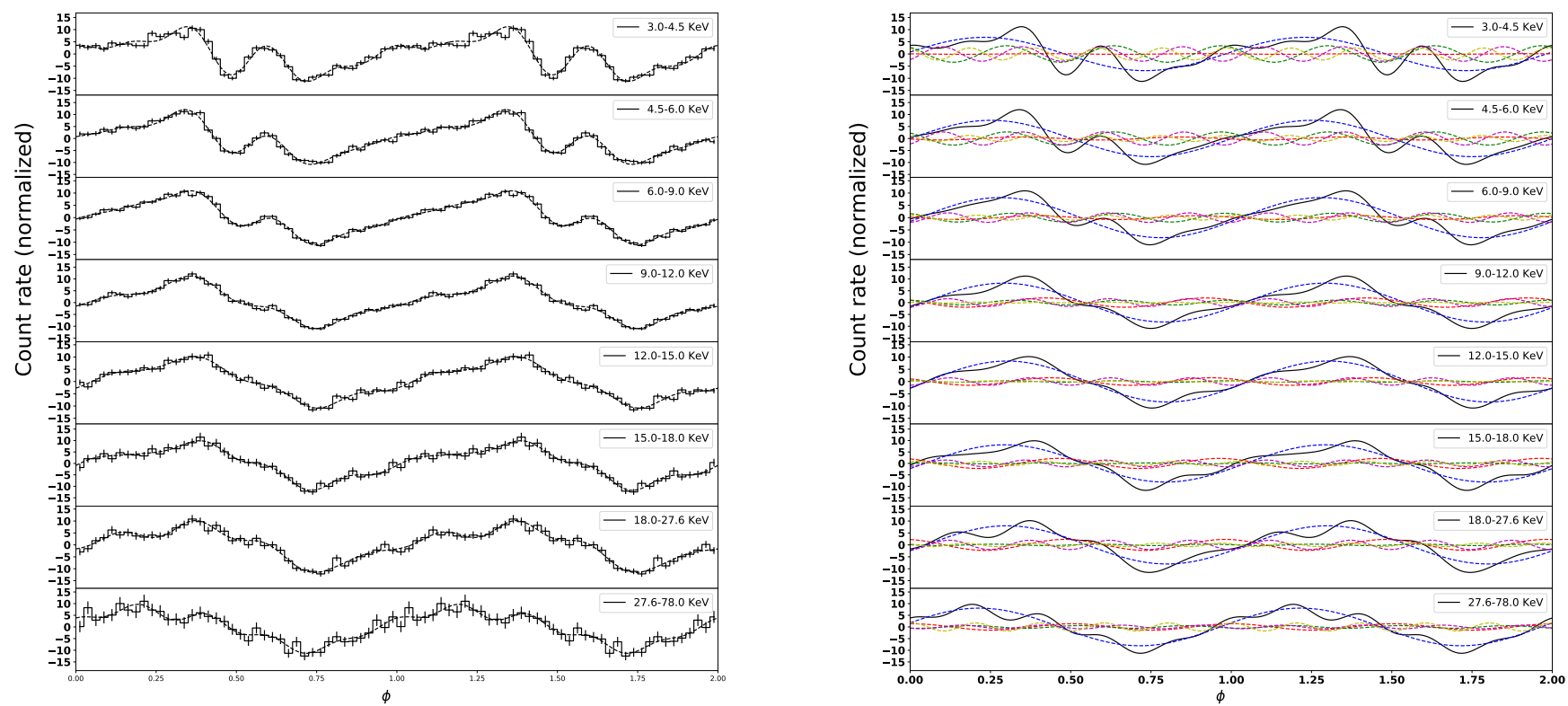

Fig. A.30. Comparison of the observed energy resolved pulse profiles of 1E1145.1-6141 and their Fourier fits at different energy bands. Left: Normalized pulse profiles of 1E1145.1-6141 at different energy ranges based on NuSTAR data. The measured profiles are represented by solid lines, whereas the Fourier fits are represented by dashed lines. The boundaries of the energy ranges are $[3,4.5,6,9,12,15$, 18, 27.6, and 78] keV. Right: Overall approximate pulse profile (black solid line) and Fourier components 1-5 (coloured dashed lines: blue, red, green, purple, and yellow, respectively). 

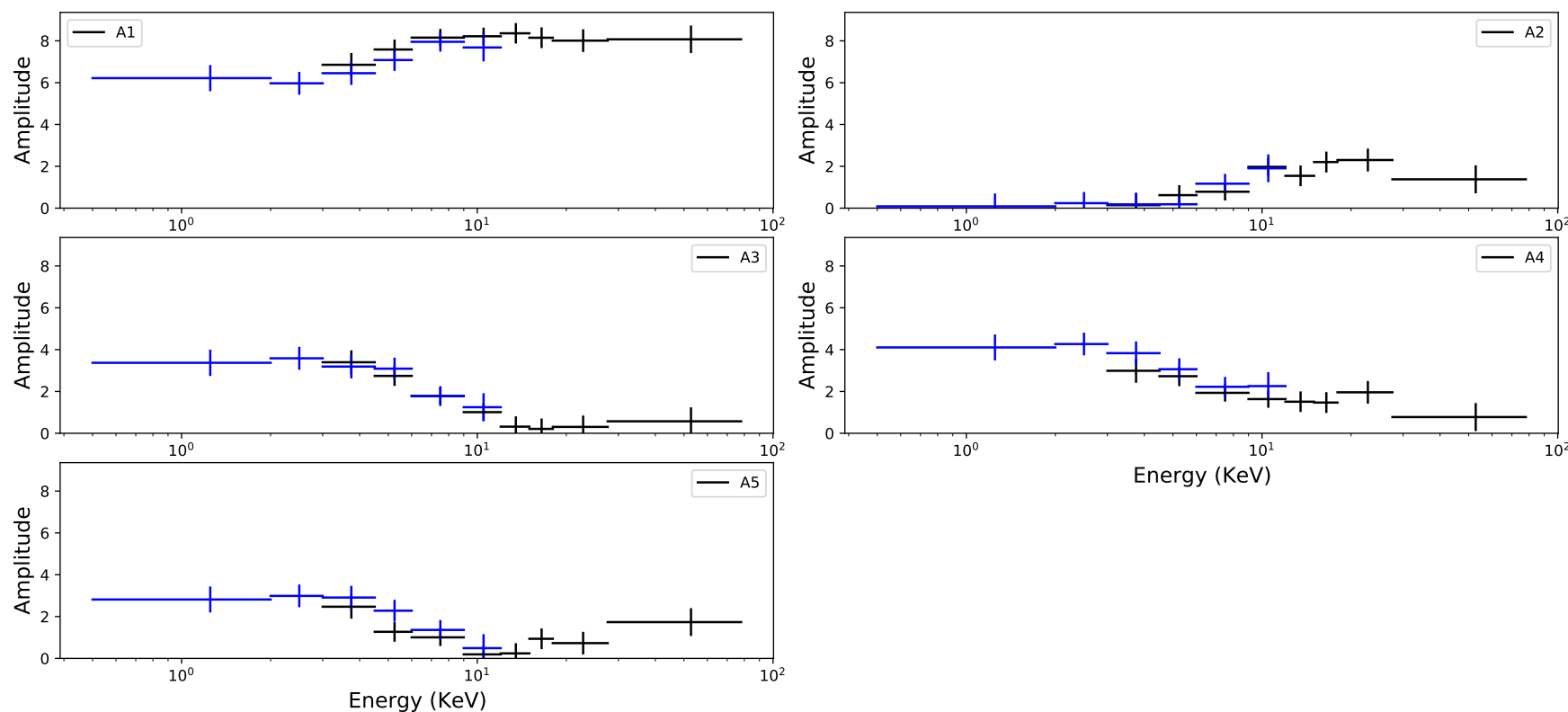

Energy $(\mathrm{KeV})$

Fig. A.31. Variation of the harmonic amplitudes for 1E1145.1-6141. The boundaries of the energy ranges are $[0.5,2,3,4.5,6,9,12,15,18,27.6$, and 78] keV. The XMM-Newton data are represented by blue markers and NuSTAR data are represented by black markers.
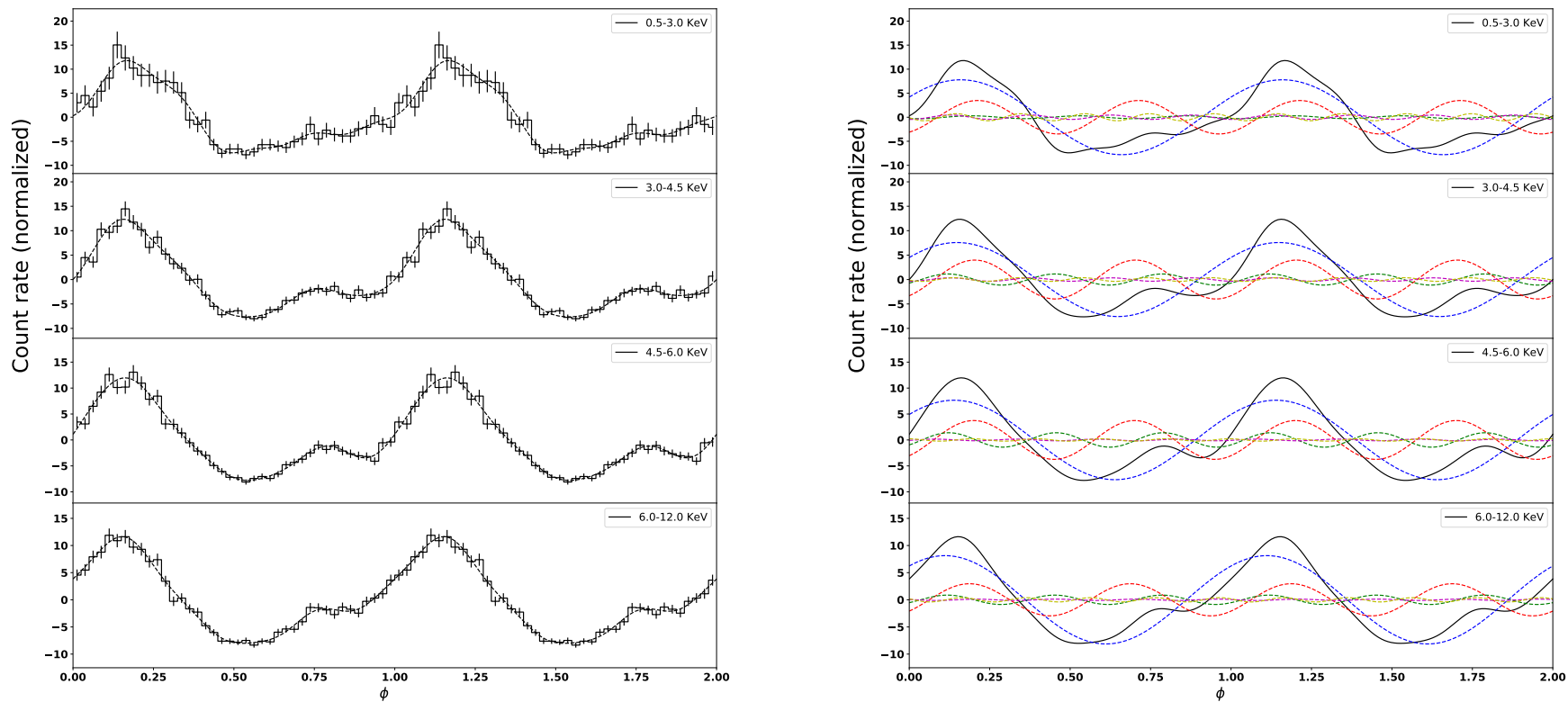

Fig. A.32. Comparison of the observed energy resolved pulse profiles of IGR J17252-3616 and their Fourier fits at different energy bands. Left: Normalized pulse profiles of IGR J17252-3616 at different energy ranges based on XMM-Newton data. The measured profiles are represented by solid lines, whereas the Fourier fits are represented by dashed lines. The boundaries of the energy ranges are [0.5, 3, 4.5, 6, 12] keV. Right: Overall approximate pulse profile (black solid line) and Fourier components 1-5 (coloured dashed lines: blue, red, green, purple, and yellow, respectively). 

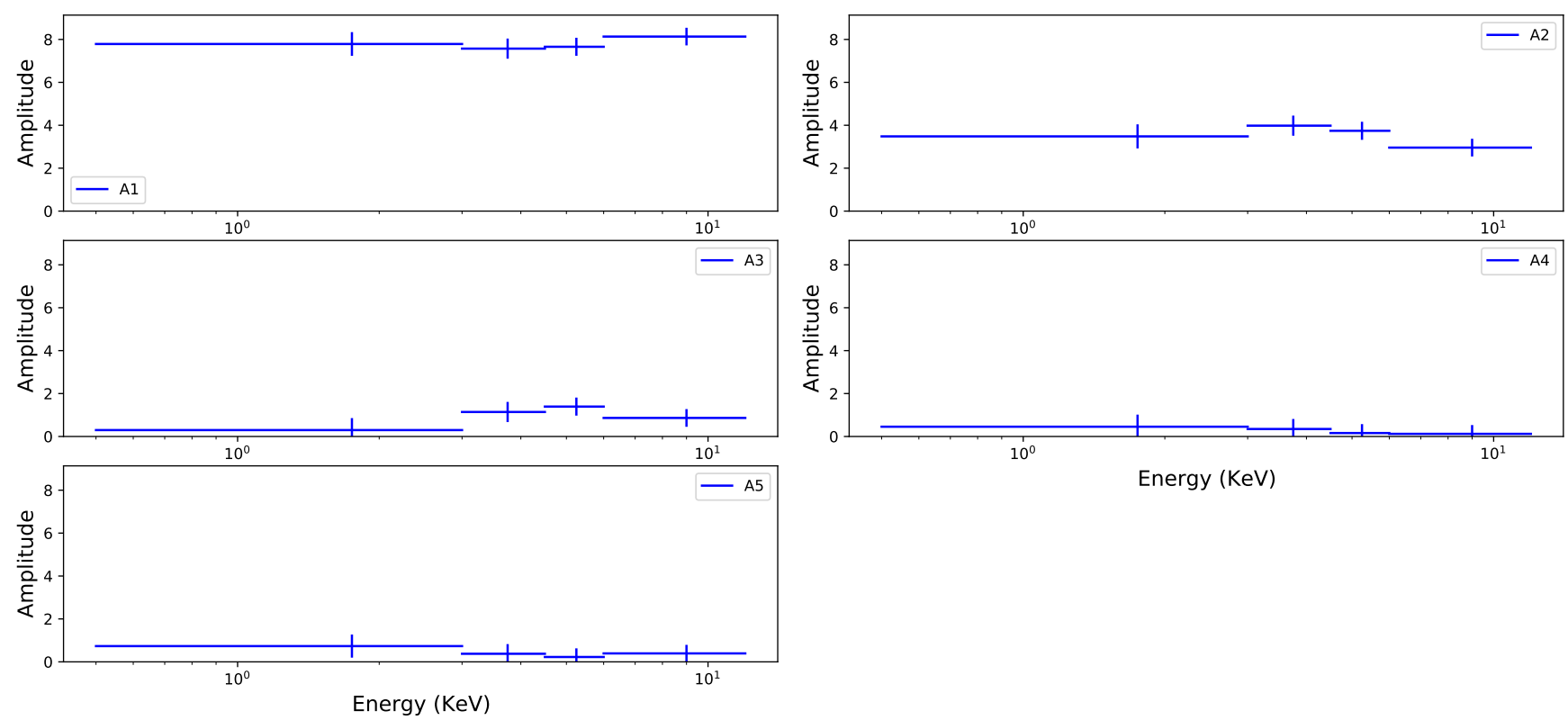

Energy $(\mathrm{KeV})$

Fig. A.33. Variation of the harmonic amplitudes for IGR J17252-3616. The boundaries of the energy ranges are $[0.5,3,4.5,6,12] \mathrm{keV}$. Only the XMM-Newton data are represented by blue markers.
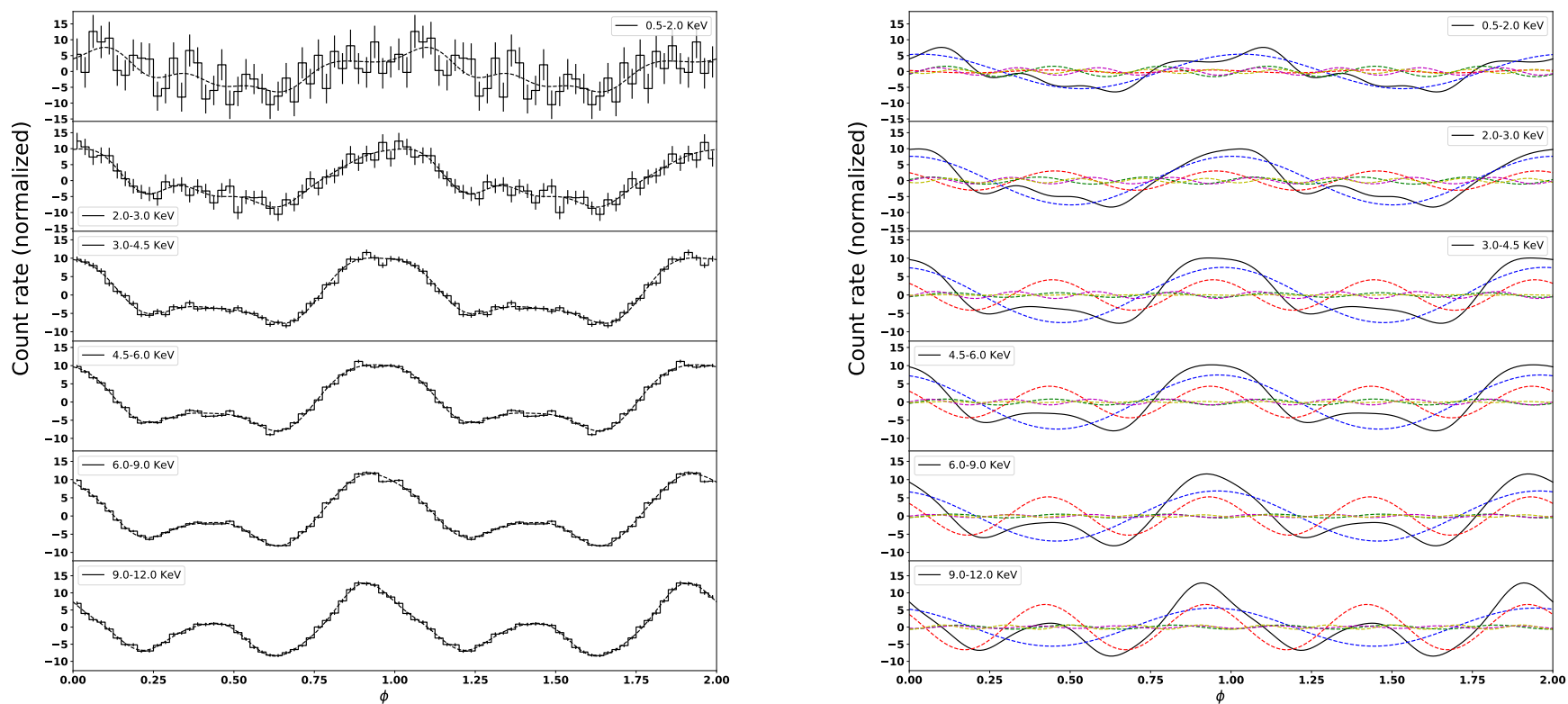

Fig. A.34. Comparison of the observed energy resolved pulse profiles of GX301-2 and their Fourier fits at different energy bands. Left: Normalized pulse profiles of GX301-2 at different energy ranges based on XMM-Newton data. The measured profiles are represented by solid lines, whereas the Fourier fits are represented by dashed lines. The boundaries of the energy ranges are $[0.5,2,3,4.5,6,9,12]$ keV. Right: Overall approximate pulse profile (black solid line) and Fourier components 1-5 (coloured dashed lines: blue, red, green, purple, and yellow, respectively). 

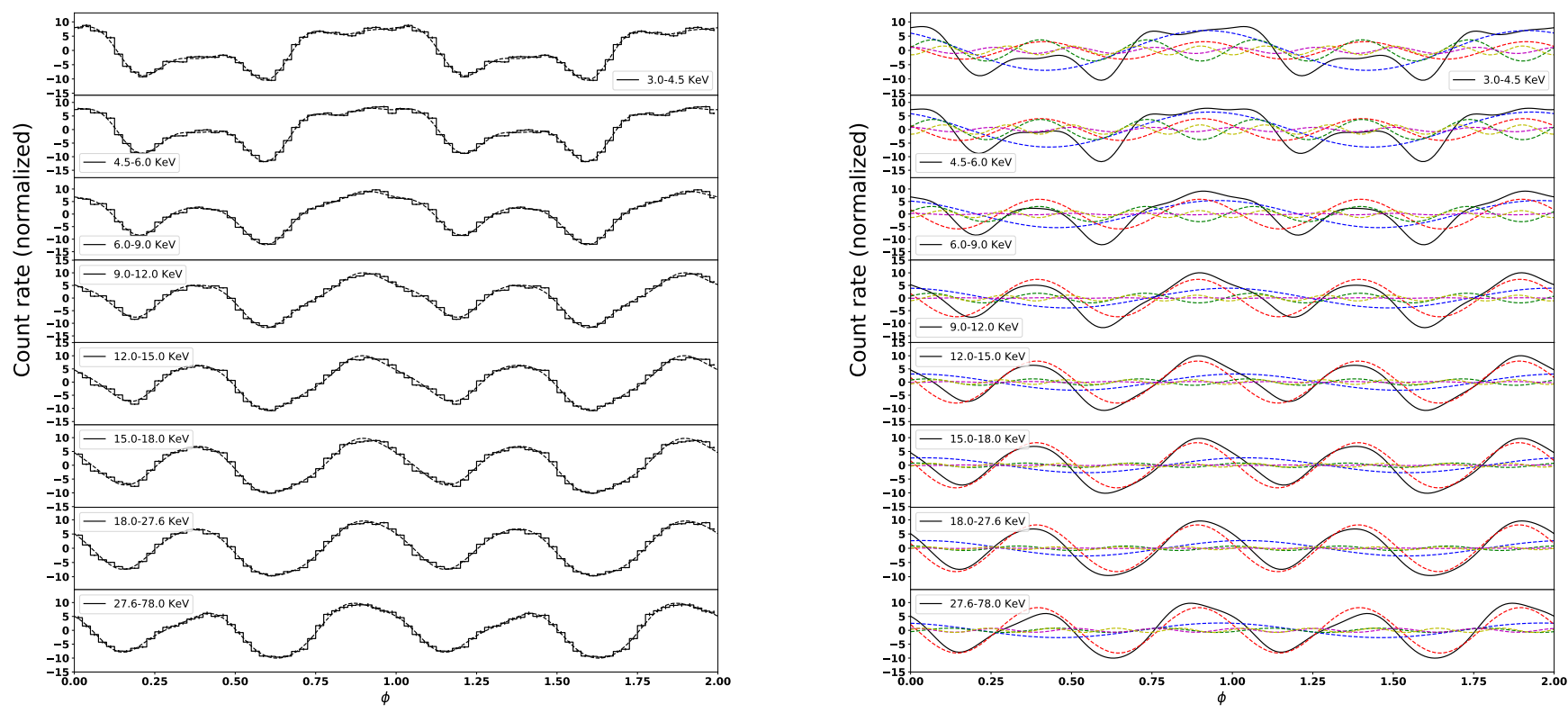

Fig. A.35. Comparison of the observed energy resolved pulse profiles of GX301-2 and their Fourier fits at different energy bands. Left: Normalized pulse profiles of GX301-2 at different energy ranges based on NuSTAR data. The measured profiles are represented by solid lines, whereas the Fourier fits are represented by dashed lines. The boundaries of the energy ranges are $[3,4.5,6,9,12,15,18,27.6$, and 78] keV. Right: Overall approximate pulse profile (black solid line) and Fourier components 1-5 (coloured dashed lines: blue, red, green, purple, and yellow, respectively).
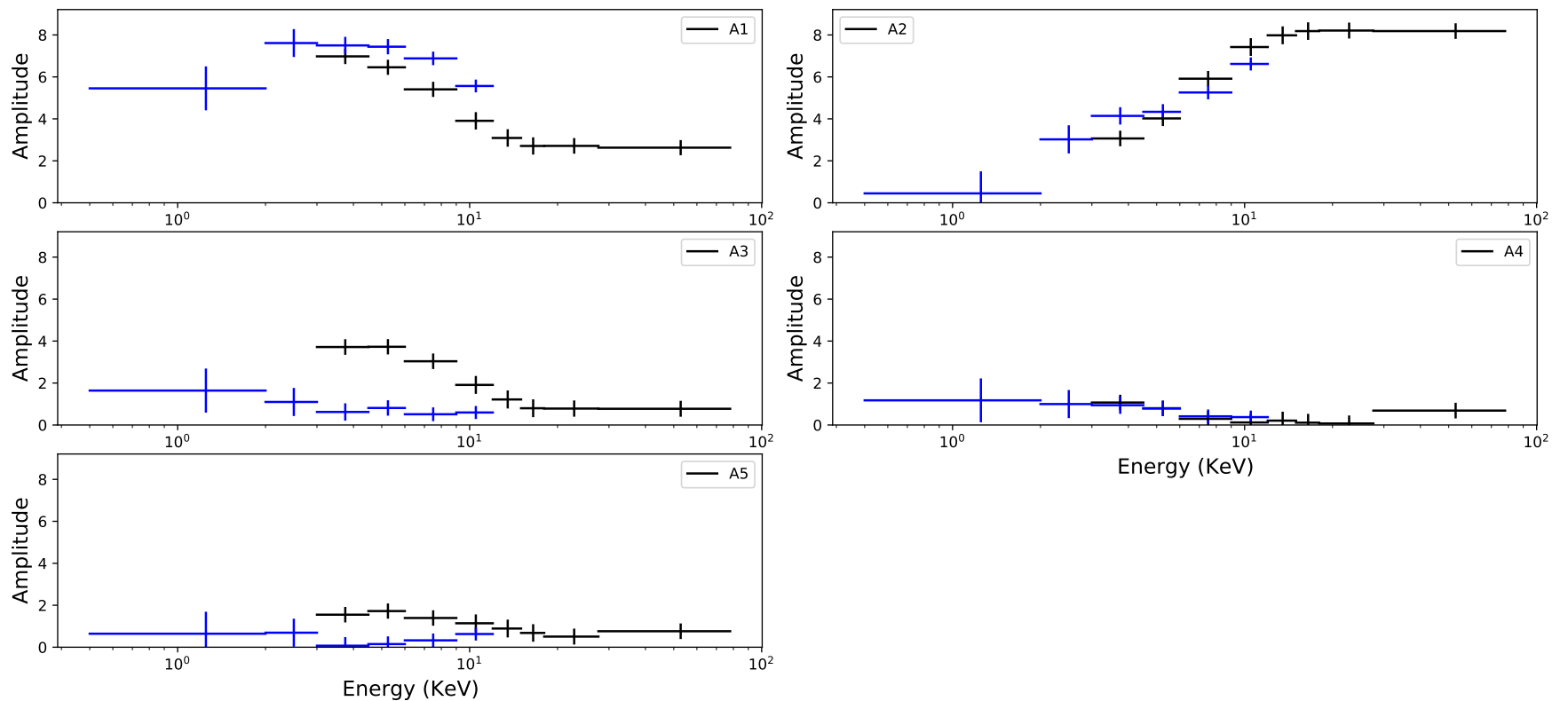

Energy $(\mathrm{KeV})$

Fig. A.36. Variation of the harmonic amplitudes for GX301-2. The boundaries of the energy ranges are $[0.5,2,3,4.5,6,9,12,15,18,27.6$, and $78] \mathrm{keV}$. The XMM-Newton data are represented by blue markers and NUSTAR data are represented by black markers. 

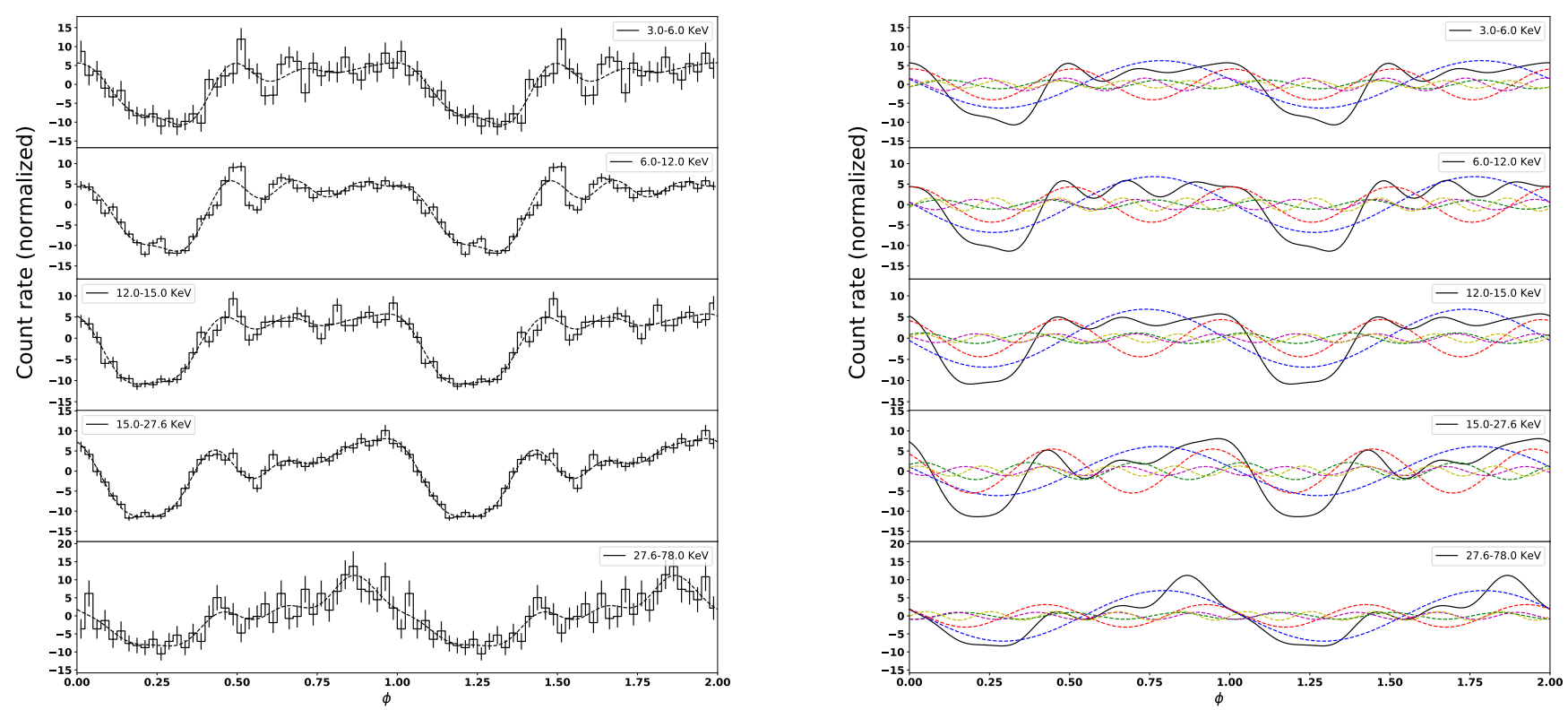

Fig. A.37. Comparison of the observed energy resolved pulse profiles of IGR J16393-4643 and their Fourier fits at different energy bands. Left: Normalized pulse profiles of IGR J16393-4643 at different energy ranges based on NuSTAR data. The measured profiles are represented by solid lines, whereas the Fourier fits are represented by dashed lines. The boundaries of the energy ranges are [3, 6, 12, 15, 27.6, and 78] keV. Right: Overall approximate pulse profile (black solid line) and Fourier components 1-5 (coloured dashed lines: blue, red, green, purple, and yellow, respectively).
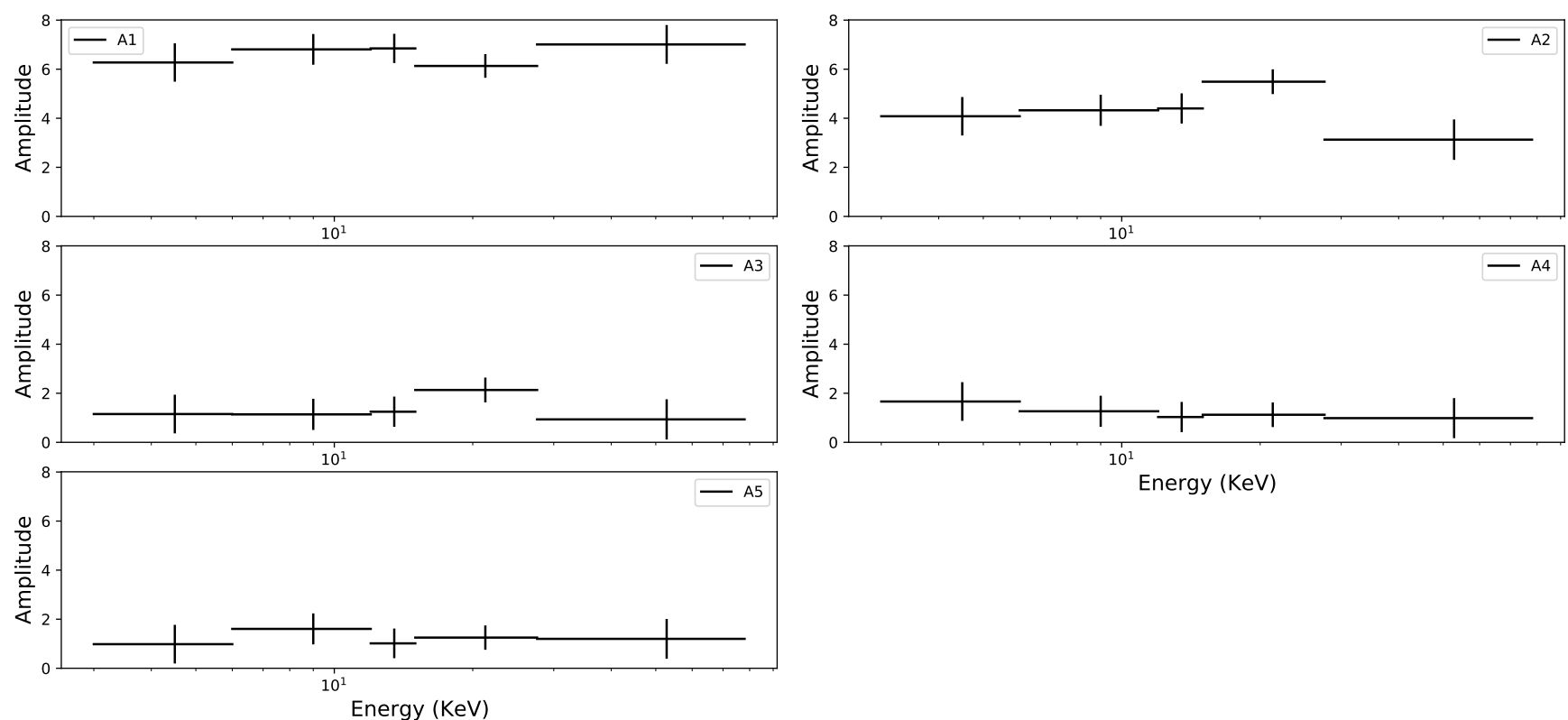

Energy $(\mathrm{KeV})$

Fig. A.38. Variation of the harmonic amplitudes for IGR J16393-4643. The boundaries of the energy ranges are [3, 6, 12, 15, 27.6, and 78] keV. Only the NUSTAR data are represented by black markers. 